\title{
Numerical aspects of a real-time sub-structuring technique in structural dynamics
}

\author{
R. Sajeeb ${ }^{\S}$, D. Roy ${ }^{\mathbb{I}}$ and C. S. Manohar ${ }^{*, \dagger, \ddagger}$ \\ Structures Lab, Department of Civil Engineering, Indian Institute of Science, Bangalore 560012, India
}

\begin{abstract}
SUMMARY
A time domain coupling technique, involving combined computational and experimental modelling, for vibration analysis of structures built-up of linear/non-linear substructures is developed. The study permits, in principle, one or more of the substructures to be modelled experimentally with measurements being made only on the interfacial degrees of freedom. The numerical and experimental substructures are allowed to communicate in real time within the present framework. The proposed strategy involves a two-stage scheme: the first is iterative in nature and is implemented at the initial stages of the solution in a non-real-time format; the second is non-iterative, employs an extrapolation scheme and proceeds in real time. Issues on time delays during communications between different substructures are discussed. An explicit integration procedure is shown to lead to solutions with high accuracy while retaining path sensitivity to initial conditions. The stability of the integration scheme is also discussed and a method for numerically dissipating the temporal growth of high-frequency errors is presented. For systems with nonlinear substructures, the integration procedure is based on a multi-step transversal linearization method; and, to account for time delays, we employ a multi-step extrapolation scheme based on the reproducing kernel particle method. Numerical illustrations on a few low-dimensional vibrating structures are presented and these examples are fashioned after problems of seismic qualification testing of engineering structures using real-time substructure testing techniques. Copyright (c) 2007 John Wiley \& Sons, Ltd.
\end{abstract}

Received 14 June 2006; Revised 28 January 2007; Accepted 10 February 2007

KEY WORDS: real-time sub-structuring; numerical and experimental sub-structures; extrapolations; reproducing kernel particle method; explicit and implicit time-stepping schemes

\footnotetext{
*Correspondence to: C. S. Manohar, Structures Lab, Department of Civil Engineering, Indian Institute of Science, Bangalore 560012, India.

${ }^{\dagger}$ E-mail: manohar@ civil.iisc.ernet.in

$\ddagger$ Professor.

$\S$ Research Student.

IAssociate Professor.

Contract/grant sponsor: Indian Institute of Science
}

Copyright (C) 2007 John Wiley \& Sons, Ltd. 


\section{INTRODUCTION}

The concept of sub-structuring finds wide applications in computational, experimental and combined computational and experimental modelling of complex vibrating structures $[1,2]$. Towards developing modal and response function models for complex linear vibrating systems, sub-structuring methods are often employed in the frequency domain and they have been the subject of extensive studies in the literature [3]. For qualification testing of structures against vibratory loads, substructuring methods in the time domain are less widely studied. Nevertheless, they are recognized as offering several advantages [4]. In these studies the structure under investigation is divided into two substructures: whilst one is modelled numerically, the other is modelled experimentally. A key feature of this substructuring scheme is that the two substructures are coupled and analysed concurrently in real time. It is this class of problems, in particular those associated with qualification testing of structures and equipments under earthquake loads, that forms the focus of this study.

The works of Takanashi and Nakashima [5], Severn et al. [6], Nakashima [7], Krawinkler [8], Williams and Blakeborough [4], provide comprehensive overviews on contemporary research related to laboratory testing of structures under earthquake loads. A brief summary of relevant issues, based on a study of these and other related works, is as follows. The methods for laboratory testing of structures under earthquake loads can be grouped as shake table tests, effective force tests, hybrid tests, pseudodynamic (PsD) tests, and tests that involve real-time substructures (RTSs). In shake table tests, the table is under displacement/acceleration control, and the test structure is excited by a vector of support motions; consequently, the test realistically simulates the spatial distribution of inertial forces. Support motions are defined a priori and are independent of the structural response. These support motions are fashioned after realistic strong ground motions. The test structure, however, often needs to be geometrically scaled down, which compromises the ability of the test to accurately represent non-linear structural behaviour and to capture the details of local structural behaviour, such as local buckling and bond failure. The effective force test is based on the observation that earthquake ground accelerations are predominantly horizontal and their effect is to cause a set of horizontal forces on the structure [9-12]. If the structural mass elements are concentrated at a few points, as in the slabs of a building frame, the equivalent inertial forces may be applied by using a few dynamic actuators. The test hardware here consists of a reaction wall and strong floor with dynamic actuators under force control. The structure to be tested is fixed to the strong floor and subjected to lateral dynamic loads. The need for geometric scaling of the structure to be tested is less severe here. Hybrid tests $[13,14]$ are a combination of shake table and effective force tests with the effect of earthquake simulated partly as a support motion and partly as a set of horizontal forces. The test requires a table under displacement/acceleration control and dynamic actuators under force control.

The shake table, effective force and hybrid tests do not readily permit studies into evolutions of failure sequences since the test duration coincides with the duration of realistic earthquake events. A PsD test combines numerical and experimental modelling procedures, wherein inertial and damping properties are simulated numerically and structural stiffness is measured experimentally $[5,9,15,16]$. Earthquake-induced structural displacements are computed numerically and applied to the structure in a static manner. Thus, the time variable in the test is slowed down and, consequently, the test can be carried out with actuators capable of applying only controlled static displacements. This would also imply that time-dependent behaviour, such as those associated with damping, rate-dependent stress-strain laws and behaviour of active elements, if any, cannot 
be simulated in the test. On the other hand, as the test proceeds, it affords a detailed examination of evolution of failure pattern in the structure.

The RTS tests aim to overcome the problems of spatial and/or temporal scaling associated with PsD and shake table tests. They are applicable to situations in which the test structure can be divided into parts: one for which the structural behaviour is well understood and, therefore, is amenable to reliable numerical modelling, and, another that displays complicated structural behaviour and thus requires to be tested experimentally. Here the numerical and experimental models are coupled: the solution of the numerical model and the testing of the experimental model take place in real time [17-31]. This strategy enables testing of structures with modest testing facilities. In principle, RTS tests can be conducted using shake tables or horizontal actuation and reaction wall systems. The development of this testing methodology has thrown open several challenges, not only in developing sophisticated control software and hardware, but also in developing numerical integration schemes for equations receiving parts of the input from experiments.

In PsD and RTS tests, time histories of earthquake-induced forces/displacements are not defined a priori: instead, they get defined via the online solution of numerical models that receive inputs from the experimental measurements; the experimental models, in turn, receive inputs based on the results of analysis of the numerical model. Errors in experiments and in numerical integration, consequently, interact in a complicated manner. Details of the testing procedure and their capabilities are crucially dependent on the integration scheme used. Several studies have examined the accuracy and stability of numerical integration schemes used in the PsD testing (see, for instance, Thewalt and Mahin [32], Combescure and Pegon [33] and Chang [34]). In RTS testing, other than questions on numerical and experimental errors, one has also to deal with the hardware and software-related time delays. These delays, for instance, could potentially lead to an erroneous detection of a hysteresis loop, which adds energy to the system leading to a possibly divergent response [18]. The central difference method has been widely used for integrating equations of motion in RTS testing [17-19,22,35]. The fact that this is an explicit integration scheme is particularly helpful in the implementation of RTS test since the need for iterations, with attendant dangers of actuator overshoots, is avoided here. On the other hand, these schemes are conditionally stable, which restricts the size of the structure and the highest testing frequency. Thus, systems with degrees of freedom (dofs) up to 12 and frequency range up to $3 \mathrm{~Hz}$ could only be studied in the work of Nakashima and Masaoka [18].

In this study, we examine issues related to numerical aspects of RTS simulations involving linear and/or non-linear substructures. Initially, we consider the case in which the substructures behave linearly and, in the sequel, we expand the scope to include non-linear substructures. A common feature is that the simulation procedure involves two phases: the first, that is iterative in nature, does not take place in real time, but serves as a precursor for launching the computations for the second phase that is non-iterative in nature and occurs in real time.

For systems with linear substructures, the numerical integration scheme is based on a state-space representation of the governing equations of motion. An explicit version of the scheme is shown to yield satisfactory solutions when applied to a set of low-dimensional oscillators under sinusoidal and samples of stationary random excitations. This solution is shown to be capable, in principle, of realizing a high order of accuracy while retaining the path sensitivity with respect to initial conditions. Questions on stability of the integration scheme are also addressed by computing the spectral radius of the amplification matrix. A numerical means to dissipate an undesirable growth of high-frequency errors is proposed and the stability properties of the resulting implicit scheme 
are also discussed. Issues related to the treatment of noise in measurements of interactive forces and in actuating the experimental substructure are not presently considered.

Subsequently, we consider situations in which one or more of numerical and/or experimental substructures behave non-linearly under applied loads. Situations of this kind are particularly of relevance in problems of earthquake engineering; wherein one aspires to design engineering structures to display specified inelastic behaviour. Blakeborough et al. [23] have developed an algorithm to approximately treat non-linearity in RTS simulations. This algorithm was able to handle nonlinearities in the stiffness characteristics of the numerical model. The present algorithm, however, is designed to handle more general forms of non-linearity and dynamical systems. The analysis of the numerical substructure is based on a multi-step transversal linearization (MTL) scheme developed earlier [36-38]. To account for time delays, which may prove to be detrimental if not handled properly in the context of non-linear structures, we propose a multi-step extrapolation strategy based on the reproducing kernel particle method (RKPM). Basically, in the existing literature, the RKPM has been developed in the context of mesh-free methods as a functional approximation scheme with or without the interpolation capability. Used as an interpolating functional approximation, the reproducing kernel (RK) scheme is devised to reproduce exact representations to polynomials up to a specified degree at any point in a given domain (see, for instance, Chen et al. [39] and $\mathrm{Li}$ et al. [40]).

Illustrative examples on linear/non-linear oscillators under support motions are presented. Even though the validation of the proposed RTS scheme is purely based on numerical sub-structuring, different substructures dynamically interact with each other only through an appropriately predicted set of interactive forces, which are, in turn, obtainable by solving for the response of these substructures as uncoupled, reduced dynamical sub-problems. This implies that in case one of the numerical substructures is later treated as a test substructure, the implementation of the RTS procedure, as outlined in this paper, remains nearly the same with the exception that parts of the interactive dynamical forces should be obtainable through an acquisition of experimental data. To begin with, however, knowledge of these interactive forces is not available. The problem is tackled through a suitable iterative algorithm so that the RTS-based solution faithfully (i.e. with a known level of accuracy) reproduces the coupled response subject to the prescribed initial conditions even during the transient regime.

\section{THE METHODOLOGY}

Towards a simple exposition of the basic idea, we consider a linear system made up of two substructures, $S^{\mathrm{I}}$ and $S^{\mathrm{II}}$, with $S^{\mathrm{I}}$ receiving multi-component support motions; see Figure 1 for a schematic sketch. We designate the subsystem $S^{\mathrm{I}}$ as the numerical substructure and $S^{\mathrm{II}}$ as the test substructure. The displacement vector $X^{\mathrm{I}}(t)$ for the substructure $S^{\mathrm{I}}$ is partitioned as $X^{\mathrm{I}}(t)=\left\{X_{i}^{\mathrm{I}}(t) X_{c}^{\mathrm{I}}(t) X_{g}^{\mathrm{I}}(t)\right\}^{\mathrm{T}}$, where, the subscripts $i, c$, and $g$, respectively, denote internal, coupling and ground dofs and the superscript $\mathrm{T}$ denotes matrix transposition. The structural mass, stiffness and damping matrices and the forcing vector consequently also get partitioned in accordance with the partitioning of the displacement vector. The governing equations of motion for $S^{\mathrm{I}}$ may be written as

$$
\left[\begin{array}{ccc}
M_{i i}^{\mathrm{I}} & M_{i c}^{\mathrm{I}} & M_{i g}^{\mathrm{I}} \\
M_{c i}^{\mathrm{I}} & M_{c c}^{\mathrm{I}} & M_{c g}^{\mathrm{I}} \\
M_{g i}^{\mathrm{I}} & M_{g c}^{\mathrm{I}} & M_{g g}^{\mathrm{I}}
\end{array}\right]\left\{\begin{array}{c}
\ddot{X}_{i}^{\mathrm{I}} \\
\ddot{X}_{c}^{\mathrm{I}} \\
\ddot{X}_{g}^{\mathrm{I}}
\end{array}\right\}+\left[\begin{array}{ccc}
C_{i i}^{\mathrm{I}} & C_{i c}^{\mathrm{I}} & C_{i g}^{\mathrm{I}} \\
C_{c i}^{\mathrm{I}} & C_{c c}^{\mathrm{I}} & C_{c g}^{\mathrm{I}} \\
C_{g i}^{\mathrm{I}} & C_{g c}^{\mathrm{I}} & C_{g g}^{\mathrm{I}}
\end{array}\right]\left\{\begin{array}{c}
\dot{X}_{i}^{\mathrm{I}} \\
\dot{X}_{c}^{\mathrm{I}} \\
\dot{X}_{g}^{\mathrm{I}}
\end{array}\right\}+\left[\begin{array}{ccc}
K_{i i}^{\mathrm{I}} & K_{i c}^{\mathrm{I}} & K_{i g}^{\mathrm{I}} \\
K_{c i}^{\mathrm{I}} & K_{c c}^{\mathrm{I}} & K_{c g}^{\mathrm{I}} \\
K_{g i}^{\mathrm{I}} & K_{g c}^{\mathrm{I}} & K_{g g}^{\mathrm{I}}
\end{array}\right]\left\{\begin{array}{c}
X_{i}^{\mathrm{I}} \\
X_{c}^{\mathrm{I}} \\
X_{g}^{\mathrm{I}}
\end{array}\right\}=\left\{\begin{array}{c}
0 \\
F_{c}(t) \\
F_{g}(t)
\end{array}\right\}
$$




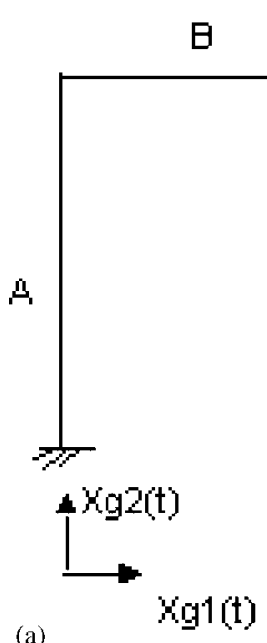

(a)

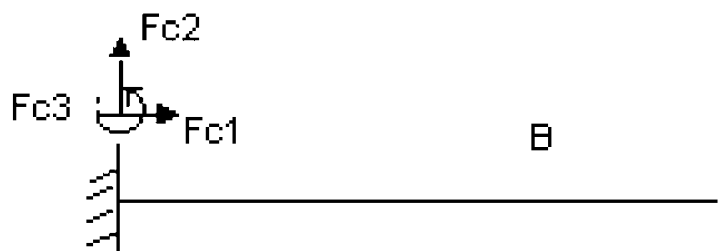

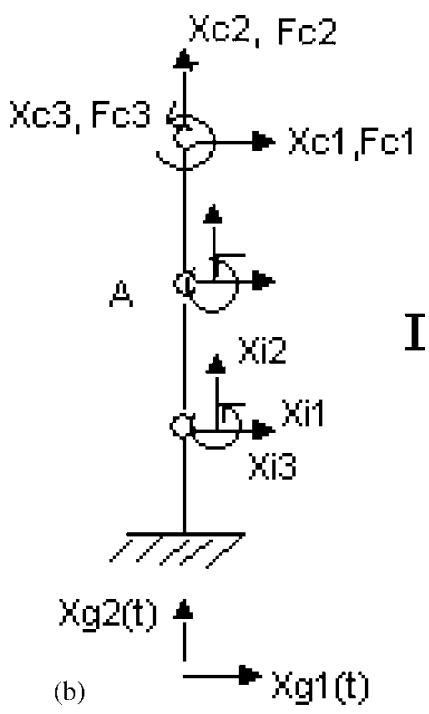

II

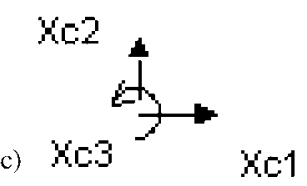

Figure 1. Schematic of RTS simulation: (a) built-up structure under support motions to be analysed; (b) numerical substructure; (c) test substructure; $x_{\mathrm{c} 1}, x_{\mathrm{c} 2}, x_{\mathrm{c} 3}=$ displacement at the coupling point obtained from the analysis of numerical substructure; $F_{\mathrm{c} 1}, F_{\mathrm{c} 2}, F_{\mathrm{c} 3}=$ forces at the coupling point obtained as reactions from the 'experimental' study of the test substructure; $x_{g_{1}}(t), x_{g_{2}}(t)=$ applied support displacements.

Here $X_{g}^{\mathrm{I}}(t)$ are the applied support displacements, $\left\{X_{i}^{\mathrm{I}}(t) X_{c}^{\mathrm{I}}(t)\right\}^{\mathrm{T}}$ is the unknown displacement vector and $F_{g}(t)$ is the vector of unknown reactions; $F_{c}(t)$ is the vector of coupling forces that are obtained as dynamic reactions from the test substructure. Equations for the unknown displacements may thus be written as

$$
\begin{gathered}
{\left[\begin{array}{cc}
M_{i i}^{\mathrm{I}} & M_{i c}^{\mathrm{I}} \\
M_{c i}^{\mathrm{I}} & M_{c c}^{\mathrm{I}}
\end{array}\right]\left\{\begin{array}{c}
\ddot{X}_{i}^{\mathrm{I}} \\
\ddot{X}_{c}^{\mathrm{I}}
\end{array}\right\}+\left[\begin{array}{cc}
C_{i i}^{\mathrm{I}} & C_{i c}^{\mathrm{I}} \\
C_{c i}^{\mathrm{I}} & C_{c c}^{\mathrm{I}}
\end{array}\right]\left\{\begin{array}{c}
\dot{X}_{i}^{\mathrm{I}} \\
\dot{X}_{c}^{\mathrm{I}}
\end{array}\right\}+\left[\begin{array}{cc}
K_{i i}^{\mathrm{I}} & K_{i c}^{\mathrm{I}} \\
K_{c i}^{\mathrm{I}} & K_{c c}^{\mathrm{I}}
\end{array}\right]\left\{\begin{array}{c}
X_{i}^{\mathrm{I}} \\
X_{c}^{\mathrm{I}}
\end{array}\right\}=\left\{F_{s}(t)\right\}+\left\{\begin{array}{c}
0 \\
F_{c}(t)
\end{array}\right\}} \\
\left\{F_{s}(t)\right\}=-\left[\begin{array}{c}
M_{i g}^{\mathrm{I}} \\
M_{c g}^{\mathrm{I}}
\end{array}\right]\left\{\ddot{X}_{g}^{\mathrm{I}}\right\}-\left[\begin{array}{c}
C_{i g}^{\mathrm{I}} \\
C_{c g}^{\mathrm{I}}
\end{array}\right]\left\{\dot{X}_{g}^{\mathrm{I}}\right\}-\left[\begin{array}{c}
K_{i g}^{\mathrm{I}} \\
K_{c g}^{\mathrm{I}}
\end{array}\right]\left\{X_{g}^{\mathrm{I}}\right\}
\end{gathered}
$$


Presently allowing both $S^{\mathrm{I}}$ and $S^{\mathrm{II}}$ to be numerical substructures, the governing equation for $S^{\mathrm{II}}$ may be written as

$$
\left[\begin{array}{cc}
M_{i i}^{\mathrm{II}} & M_{i c}^{\mathrm{II}} \\
M_{c i}^{\mathrm{II}} & M_{c c}^{\mathrm{II}}
\end{array}\right]\left\{\begin{array}{c}
\ddot{X}_{i}^{\mathrm{II}} \\
\ddot{X}_{c}^{\mathrm{II}}
\end{array}\right\}+\left[\begin{array}{cc}
C_{i i}^{\mathrm{I}} & C_{i c}^{\mathrm{II}} \\
C_{c i}^{\mathrm{II}} & C_{c c}^{\mathrm{II}}
\end{array}\right]\left\{\begin{array}{c}
\dot{X}_{i}^{\mathrm{II}} \\
\dot{X}_{c}^{\mathrm{II}}
\end{array}\right\}+\left[\begin{array}{cc}
K_{i i}^{\mathrm{II}} & K_{i c}^{\mathrm{II}} \\
K_{c i}^{\mathrm{II}} & K_{c c}^{\mathrm{II}}
\end{array}\right]\left\{\begin{array}{c}
X_{i}^{\mathrm{II}} \\
X_{c}^{\mathrm{II}}
\end{array}\right\}=\left\{\begin{array}{c}
0 \\
F_{c}(t)
\end{array}\right\}
$$

From the conditions of compatibility at the interface between $S^{\mathrm{I}}$ and $S^{\mathrm{II}}$, one gets $X_{c}^{\mathrm{I}}(t)=X_{c}^{\mathrm{II}}(t)$. It is assumed that the test substructure does not receive any excitations other than displacement inputs transmitted by the numerical substructure through the coupling dofs. Furthermore, we note that $X_{c}^{\mathrm{I}}(t)$ obtained from the solution of Equation (2) serves as 'support motions' to the substructure $S^{\mathrm{II}}$ and the reactions $F_{c}(t)$ obtained through Equation (3) gets fed back to Equation (2). For further discussion we introduce the notation $X^{\mathrm{I}}=\left\{X_{i}^{\mathrm{I}} X_{c}^{\mathrm{I}}\right\}^{\mathrm{T}}$ and $X^{\mathrm{II}}=\left\{X_{i}^{\mathrm{II}}\right\}$. One can show that the equation of motion for the system built-up of $S^{\mathrm{I}}$ and $S^{\mathrm{II}}$ may be arranged as

$$
\left[\begin{array}{cc}
M^{\mathrm{I}} & M^{12} \\
M^{21} & M^{\mathrm{II}}
\end{array}\right]\left\{\begin{array}{l}
\ddot{X}^{\mathrm{I}} \\
\ddot{X}^{\mathrm{II}}
\end{array}\right\}+\left[\begin{array}{cc}
C^{\mathrm{I}} & C^{12} \\
C^{21} & C^{\mathrm{II}}
\end{array}\right]\left\{\begin{array}{c}
\dot{X}^{\mathrm{I}} \\
\dot{X}^{\mathrm{II}}
\end{array}\right\}+\left[\begin{array}{cc}
K^{\mathrm{I}} & K^{12} \\
K^{21} & K^{\mathrm{II}}
\end{array}\right]\left\{\begin{array}{c}
X^{\mathrm{I}} \\
X^{\mathrm{II}}
\end{array}\right\}=\left\{\begin{array}{c}
F^{\mathrm{I}}(t) \\
F^{\mathrm{II}}(t)
\end{array}\right\}
$$

Let $X^{\mathrm{I}}(t) \in \mathbf{R}^{n_{1}}$ and $X^{\mathrm{II}}(t) \in \mathbf{R}^{n_{2}}$, so that the structural matrices are $M^{\mathrm{I}}, C^{\mathrm{I}}, K^{\mathrm{I}} \in \mathbf{R}^{n_{1} \times n_{1}}$ and $M^{\mathrm{II}}, C^{\mathrm{II}}, K^{\mathrm{II}} \in \mathbf{R}^{n_{2} \times n_{2}}$. The cross-coupling matrices $M^{12}, C^{12}, K^{12} \in \mathscr{R}^{n_{1} \times n_{2}}$ and $M^{21}, C^{21}$, $K^{21} \in \mathscr{R}^{n_{2} \times n_{1}}$ yield the interactive forces acting on the boundaries between the two substructures. Incidentally, in case one uses the finite element discretization and an additional assumption on proportional damping to obtain the $[M],[C]$ and $[K]$ matrices in Equation (4), it often turns out that these matrices are symmetric so that $M^{21}=\left[M^{12}\right]^{\mathrm{T}}, C^{21}=\left[C^{12}\right]^{\mathrm{T}}$ and $K^{21}=\left[K^{12}\right]^{\mathrm{T}}$. However, the present RTS algorithm does not impose such constraints on the coefficient matrices.

Since both $S^{\mathrm{I}}$ and $S^{\mathrm{II}}$ are presently numerical substructures, all the elements of the matrices here are numerically computable. The objective of the RTS-based simulation in this case would be to solve for the substructural equations of motion and then to combine these solutions so as to reconstruct the response of the structure built of $S^{\mathrm{I}}$ and $S^{\mathrm{II}}$. Moreover, such a reconstruction has to be performed in a 'path-sensitive' manner so as to correctly capture the dependence of solutions to initial conditions. Let the time interval $\left(t_{0}, T \mid t_{0}<T\right]$ be ordered as $t_{0}<t_{1}<\cdots<t_{N}=T$ into $N$ intervals with $h_{i}=t_{i}-t_{i-1}, i=1,2, \ldots, N$. The equations of motion for $S^{\mathrm{I}}$ may now be written as

$$
M^{\mathrm{I}} \ddot{X}^{\mathrm{I}}+C^{\mathrm{I}} \dot{X}^{\mathrm{I}}+K^{\mathrm{I}} X^{\mathrm{I}}=F^{\mathrm{I}}(t)-G^{\mathrm{I}}\left(\ddot{X}^{\mathrm{II}}, \dot{X}^{\mathrm{II}}, X^{\mathrm{II}}\right)
$$

The interactive forcing vector $G^{\mathrm{I}}$ is given by

$$
G^{\mathrm{I}}\left(\ddot{X}^{\mathrm{II}}, \dot{X}^{\mathrm{II}}, X^{\mathrm{I}}\right)=-M^{12} \ddot{X}^{\mathrm{II}}-C^{12} \dot{X}^{\mathrm{I}}-K^{12} X^{\mathrm{I}}
$$

Let the initial condition vector for $S^{\mathrm{I}}$ be $\left(\left(X_{0}^{\mathrm{I}}\right)^{\mathrm{T}},\left(\dot{X}_{0}^{\mathrm{I}}\right)^{\mathrm{T}}\right)^{\mathrm{T}}$ and that for $S^{\mathrm{II}}$ be $\left(\left(X_{0}^{\mathrm{II}}\right)^{\mathrm{T}},\left(\dot{X}_{0}^{\mathrm{II}}\right)^{\mathrm{T}}\right)^{\mathrm{T}}$. We now need to integrate Equation (5) over the interval $\left(t_{0}, t_{1}\right]$. This, however, is not straightforward since $G^{\mathrm{I}}\left(\ddot{X}^{\mathrm{II}}, \dot{X}^{\mathrm{II}}, X^{\mathrm{I}}\right)$ is not known except at $t=t_{0}$ where it is given by $G^{\mathrm{I}, 0}\left(\ddot{X}_{0}^{\mathrm{II}}, \dot{X}_{0}^{\mathrm{II}}, X_{0}^{\mathrm{II}}\right)$. One could possibly use an approximation

$$
G^{\mathrm{I}}\left(\ddot{X}^{\mathrm{II}}, \dot{X}^{\mathrm{II}}, X^{\mathrm{I}} \mid t \in\left(t_{0}, t_{1}\right]\right) \cong G^{\mathrm{I}, 0}
$$

uniformly over $\left(t_{0}, t_{1}\right]$. To understand the nature of the resulting error we expand $G^{\mathrm{I}}\left(\ddot{X}^{\mathrm{II}}, \dot{X}^{\mathrm{II}}, X^{\mathrm{I}}\right)$ in a Taylor series based at $t=t_{0}$

$$
G^{\mathrm{I}}\left(\ddot{X}^{\mathrm{II}}, \dot{X}^{\mathrm{II}}, X^{\mathrm{I}} \mid t \in\left(t_{0}, t_{1}\right]\right)=G^{\mathrm{I}, 0}+E^{\mathrm{I}, 0}(t)
$$


The error $E^{\mathrm{I}, 0}(t)$ is given by

$$
E^{\mathrm{I}, 0}(t)=\sum_{j=1}^{n_{1}}\left(\frac{\partial G^{\mathrm{I}}}{\partial \ddot{X}_{t, j}^{\mathrm{II}}} \dddot{X}_{t, j}^{\mathrm{II}}+\frac{\partial G^{\mathrm{I}}}{\partial \dot{X}_{t, j}^{\mathrm{II}}} \ddot{X}_{t, j}^{\mathrm{II}}+\frac{\partial G^{\mathrm{I}}}{\partial X_{t, j}^{\mathrm{II}}} \dot{X}_{t, j}^{\mathrm{II}}\right)\left(t-t_{0}\right)+O\left(t-t_{0}\right)^{2}
$$

where $X_{t, j}^{\mathrm{II}} \in \mathscr{R}$ denotes the $j$ th element of the displacement vector $X^{\mathrm{II}}(t)$. This leads to an unacceptably high $O\left(h_{1}\right)$ error for the interactive forcing function over $\left(t_{0}, t_{1}\right]$. At this stage, we project the sub-structural Equations (5) in the state-space form as

$$
\begin{aligned}
& \dot{X}^{\mathrm{I}}=Y^{\mathrm{I}} \\
& \dot{Y}^{\mathrm{I}}=-C^{\mathrm{I}} Y^{\mathrm{I}}-K^{\mathrm{I}} X^{\mathrm{I}}+F^{\mathrm{I}}(t)+G^{\mathrm{I}}\left(\hat{X}^{\mathrm{II}}\right)
\end{aligned}
$$

where $X^{\mathrm{I}}(t), Y^{\mathrm{I}}(t)$ are, respectively, the displacement and velocity components of the substructure $S^{\mathrm{I}}$. We introduce notations $\hat{X}^{\mathrm{I}}(t)=\left(\left(X^{\mathrm{I}}\right)^{\mathrm{T}},\left(Y^{\mathrm{I}}\right)^{\mathrm{T}}\right)^{\mathrm{T}} \in \mathscr{R}^{2 n_{1}}$ and $\hat{X}^{\mathrm{II}}(t)=\left(\left(X^{\mathrm{II}}\right)^{\mathrm{T}},\left(Y^{\mathrm{II}}\right)^{\mathrm{T}}\right)^{\mathrm{T}} \in \mathscr{R}^{2 n_{2}}$ that are, respectively, $2 n_{1}$ - and $2 n_{2}$-dimensional state vectors for $S^{\mathrm{I}}$ and $S^{\mathrm{II}}$. Note that the acceleration vectors $\ddot{X}^{\mathrm{I}}$ and $\ddot{X}^{\mathrm{II}}$ are expressible in terms of their corresponding displacement and velocity vectors and thus the interactive forcing $G^{\mathrm{I}}\left(\hat{X}^{\mathrm{II}}\right)$ may be thought of as a function of $\hat{X}^{\mathrm{II}}$ only. To derive an explicit integration scheme at the sub-structural level such that integration of one of the sub-structural equations of motion is performed without a complete knowledge of the response of other substructures over the same time interval, we begin by integrating Equation (5) via the uniform approximation $G^{\mathrm{I}}\left(\hat{X}^{\mathrm{II}}\right) \approx G^{\mathrm{I}, 0}$. We subsequently improve the approximate solution through iterations. Thus, the first iterate for the solution of Equation (5) for $t \in\left(t_{0}, t_{1}\right]$ may be written as

$$
\begin{aligned}
\hat{X}^{\mathrm{I}, 1}\left(t \mid t \in\left(t_{0}, t_{1}\right]\right)= & \exp \left[A^{\mathrm{I}}\left(t-t_{0}\right)\right] \hat{X}_{0}^{\mathrm{I}}+\exp \left[A^{\mathrm{I}}\left(t-t_{0}\right)\right] \\
& \times \int_{t_{0}}^{t} \exp \left[-A^{\mathrm{I}}\left(s-t_{0}\right)\right]\left\{\hat{F}^{\mathrm{I}}(s)+\hat{G}^{\mathrm{I}, 0}\right\} \mathrm{d} s
\end{aligned}
$$

$\hat{X}^{\mathrm{I}, 1}=\left(\left(X^{\mathrm{I}, 1}\right)^{\mathrm{T}},\left(\dot{X}^{\mathrm{I}, 1}\right)^{\mathrm{T}}\right)^{\mathrm{T}} \in \mathscr{R}^{2 n_{1}}$ is the first iterate of the state vector corresponding to $S^{\mathrm{I}}$ and $A^{\mathrm{I}} \in \mathscr{R}^{2 n_{1} \times 2 n_{1}}$ is the associated system coefficient matrix given by

$$
A^{\mathrm{I}}=\left[\begin{array}{cc}
0 & I \\
-\left[M^{\mathrm{I}}\right]^{-1} K^{\mathrm{I}} & -\left[M^{\mathrm{I}}\right]^{-1} C^{\mathrm{I}}
\end{array}\right]
$$

with $0, I \in \mathscr{R}^{n_{1} \times n_{1}}$ being the zero and identity matrices, respectively. Moreover, $\hat{F}(t)$ and $\hat{G}^{\mathrm{I}, 0} \in \mathscr{R}^{2 n_{1}}$ are, respectively, the augmented and interactive force vectors given by

$$
\hat{F}^{\mathrm{I}}(t)=\left\{0^{\mathrm{T}},\left(F^{\mathrm{I}}(t)\right)^{\mathrm{T}}\right)^{\mathrm{T}}, \quad \hat{G}^{\mathrm{I}, 0}=\left\{0^{\mathrm{T}},\left(G^{\mathrm{I}, 0}\right)^{\mathrm{T}}\right)^{\mathrm{T}}, \quad 0 \in \mathscr{R}^{n_{1}}
$$

$\hat{X}_{0}^{\mathrm{I}}=\left\{\left(X^{\mathrm{I}}\left(t_{0}\right)\right)^{\mathrm{T}},\left(\dot{X}^{\mathrm{I}}\left(t_{0}\right)\right)^{\mathrm{T}}\right)^{\mathrm{T}}$ is the initial condition vector for the substructure $S^{\mathrm{I}}$.

Note that $\hat{X}_{0}^{\mathrm{I}}$ may also be interpreted as the 0 th iterate $\hat{X}^{\mathrm{I}, 0}$ of the solution to $S^{\mathrm{I}}$ over $\left(t_{0}, t_{1}\right]$. Before obtaining the next iterate $\hat{X}^{\mathrm{I}, 2}(t)$ for $S^{\mathrm{I}}$, a better estimate for the interactive force vector $G^{\mathrm{I}}$ must be available. This is only possible by generating a better estimate $\hat{X}^{\mathrm{II}, 1}(t)$ of the solution to the other substructure $S^{\mathrm{II}}$ over $\left(t_{0}, t_{1}\right]$. It is noted that the 0 th iterate $\hat{X}^{\mathrm{II}, 0}=\left\{\left(X^{\mathrm{II}}\left(t_{0}\right) \triangleq X_{0}^{\mathrm{II}}\right)^{\mathrm{T}}\right.$, 
$\left.\left(\dot{X}^{\mathrm{II}}\left(t_{0}\right) \triangleq \dot{X}_{0}^{\mathrm{II}}\right)^{\mathrm{T}}\right)^{\mathrm{T}}$ for $S^{\mathrm{II}}$ is already available through the known initial conditions. Thus, the equations of motion for $S^{\mathrm{II}}$ are given by

$$
\begin{aligned}
& \dot{X}^{\mathrm{II}}=Y^{\mathrm{II}} \\
& \dot{Y}^{\mathrm{II}}=-C^{\mathrm{II}} Y^{\mathrm{II}}-K^{\mathrm{II}} X^{\mathrm{II}}+F^{\mathrm{II}}(t)+G^{\mathrm{II}}\left(\hat{X}^{\mathrm{I}}\right)
\end{aligned}
$$

$G^{\mathrm{II}}\left(\hat{X}^{\mathrm{I}}\right) \in \mathscr{R}^{n_{2}}$ is the iterative force vector acting on $S^{\mathrm{II}}$ and is given by

$$
G^{\mathrm{II}}\left(\hat{X}^{\mathrm{I}}\right)=-\left[M^{\mathrm{I}}\right]^{-1}\left[M^{21} \ddot{X}^{\mathrm{I}}-C^{21} \dot{X}^{\mathrm{I}}-K^{21} X^{\mathrm{I}}\right]
$$

Since an approximation to $\left(\hat{X}^{\mathrm{I}}(t) \mid t \in\left(t_{0}, t_{1}\right]\right)$ is already available as $\hat{X}^{\mathrm{I}, 1}(t)$, one may construct the first iterate $G^{\mathrm{II}, 1}(t)$ as the following explicit function of time:

$$
G^{\mathrm{II}, 1}(t)=-\left[M^{\mathrm{I}}\right]^{-1}\left[M^{21} \ddot{X}^{\mathrm{I}, 1}(t)-C^{21} \dot{X}^{\mathrm{I}, 1}(t)-K^{21} X^{\mathrm{I}, 1}(t)\right]
$$

Now the first iterative solution for the substructure $S^{\mathrm{II}}$ over $\left(t_{0}, t_{1}\right]$ may be found as

$$
\begin{aligned}
\hat{X}^{\mathrm{II}, 1}\left(t \mid t \in\left(t_{0}, t_{1}\right]\right)= & \exp \left[A^{\mathrm{II}}\left(t-t_{0}\right)\right] \hat{X}_{0}^{\mathrm{II}}+\exp \left[A^{\mathrm{II}}\left(t-t_{0}\right)\right] \\
& \times \int_{t_{0}}^{t} \exp \left[-A^{\mathrm{II}}\left(s-t_{0}\right)\right]\left\{\hat{F}^{\mathrm{II}}(s)+\hat{G}^{\mathrm{II}, 1}\right\} \mathrm{d} s
\end{aligned}
$$

where $\hat{X}^{\mathrm{II}, 1}=\left(\left(X^{\mathrm{II}, 1}\right)^{\mathrm{T}},\left(\dot{X}^{\mathrm{II}, 1}\right)^{\mathrm{T}}\right)^{\mathrm{T}} \in \mathscr{R}^{2 n_{2}}$ is the first iterative approximation to $\hat{X}^{\mathrm{II}}=\left\{\left(X^{\mathrm{II}}\right)^{\mathrm{T}}\right.$, $\left.\left(\dot{X}^{\mathrm{II}}\right)^{\mathrm{T}}\right)^{\mathrm{T}} \in \mathscr{R}^{2 n_{2}}, A^{\mathrm{II}}$ is the coefficient matrix for $S^{\mathrm{II}}$ given by

$$
A^{\mathrm{II}}=\left[\begin{array}{cc}
0 & I \\
-\left[M^{\mathrm{II}}\right]^{-1} K^{\mathrm{II}} & -\left[M^{\mathrm{II}}\right]^{-1} C^{\mathrm{II}}
\end{array}\right] \in \mathscr{R}^{2 n_{2} \times 2 n_{2}}
$$

In the above equations, $\hat{X}^{\mathrm{II}, 0}=\hat{X}^{\mathrm{II}}\left(t_{0}\right) \triangleq \hat{X}_{0}^{\mathrm{II}}$ is the initial condition vector, $\hat{F}^{\mathrm{II}}(t)=\left\{0^{\mathrm{T}},\left(F^{\mathrm{II}}(t)\right)^{\mathrm{T}}\right)^{\mathrm{T}}$, $\hat{G}^{\mathrm{II}, 1}=\left\{0^{\mathrm{T}},\left(G^{\mathrm{II}, 1}\right)^{\mathrm{T}}\right)^{\mathrm{T}} \in \mathscr{R}^{2 n_{2}}$ are the augmented external forcing and interactive forcing vectors, respectively. Having obtained the estimate $\left(\hat{X}^{\mathrm{II}, 1}(t) \mid t \in\left(t_{0}, t_{1}\right]\right)$ of the response of $S^{\mathrm{II}}$, one may go back to the substructure $S^{\mathrm{I}}$ and use $\hat{X}^{\mathrm{II}, 1}(t)$ to obtain the next iterative estimate $\hat{G}^{\mathrm{I}, 1}(t)$ of the interactive forcing $\hat{G}^{\mathrm{I}}(t)$ acting on $S^{\mathrm{I}}$. Using Equation (9a), with $\hat{G}^{\mathrm{I}, 0}$ replaced by $\hat{G}^{\mathrm{I}, 1}(t)$, one may now determine $\hat{X}^{\mathrm{I}, 2}(t)$ of $S^{\mathrm{I}}$. This establishes an iterative procedure to generate a couple of semiinfinite sequences $\left\{\hat{X}^{\mathrm{I}, 0}, \hat{X}^{\mathrm{I}, 1}(t), \ldots\right\}$ and $\left\{\hat{X}^{\mathrm{II}, 0}, \hat{X}^{\mathrm{II}, 1}(t), \ldots\right\}$ over $\left(t_{0}, t_{1}\right]$. These sequences are meaningful provided they are convergent, so that the $\operatorname{limits}_{\lim _{k \rightarrow \infty}} \hat{X}^{\mathrm{I}, k}(t)$ and $\lim _{k \rightarrow \infty} \hat{X}^{\mathrm{II}, k}(t)$ exists and, respectively, equal $\hat{X}^{\mathrm{I}}(t)$ and $\hat{X}^{\mathrm{II}}(t)$ over the same interval.

In order to numerically investigate the convergence of the two sequences we define the Euclidean norm over $\left(t_{i-1}, t_{i}\right]$ (with $t_{i-1}<t_{i}$ ) as

$$
d=\langle\|X(t)-Y(t)\|\rangle=\left[\int_{t_{i-1}}^{t_{i}}\left\{\sum_{j=1}^{n}\left(X_{t, j}-Y_{t, j}\right)^{2}\right\} \mathrm{d} t\right]^{1 / 2}
$$

between $X(t)=\left\{X_{t, j} \mid j=1, \ldots, n\right\} \in \mathscr{R}^{n}$ and $Y(t)=\left\{Y_{t, j} \mid j=1, \ldots, n\right\} \in \mathscr{R}^{n}$. Furthermore, to see how the vector functions $\hat{X}^{\mathrm{I}, k}(t)$ and $\hat{X}^{\mathrm{II}, k}(t)$ converge for increasing $k \in \mathscr{Z}^{+}$, we obtain explicit 
expressions for these functions (using Equations (9a) and (12a)) as

$$
\begin{aligned}
\hat{X}^{\mathrm{I}, k}\left(t \mid t \in\left(t_{0}, t_{1}\right]\right)= & \exp \left[A^{\mathrm{I}}\left(t-t_{0}\right)\right] \hat{X}_{0}^{\mathrm{I}}+\exp \left[A^{\mathrm{I}}\left(t-t_{0}\right)\right] \\
& \times \int_{t_{0}}^{t} \exp \left[-A^{\mathrm{I}}\left(s-t_{0}\right)\right]\left\{\hat{F}^{\mathrm{I}}(s)+\hat{G}^{\mathrm{I}, k-1}(s)\right\} \mathrm{d} s \\
\hat{X}^{\mathrm{II}, k}\left(t \mid t \in\left(t_{0}, t_{1}\right]\right)= & \exp \left[A^{\mathrm{II}}\left(t-t_{0}\right)\right] \hat{X}_{0}^{\mathrm{II}}+\exp \left[A^{\mathrm{II}}\left(t-t_{0}\right)\right] \\
& \times \int_{t_{0}}^{t} \exp \left[-A^{\mathrm{II}}\left(s-t_{0}\right)\right]\left\{\hat{F}^{\mathrm{II}}(s)+\hat{G}^{\mathrm{II}, k}(s)\right\} \mathrm{d} s
\end{aligned}
$$

for $k=1,2, \ldots$. Thus, the only terms contributing to the difference between $\hat{X}^{\mathrm{I}, k+1}(t)$ and $\hat{X}^{\mathrm{I}, k}(t)$ (or, $\hat{X}^{\mathrm{II}, k+1}(t)$ and $\left.\hat{X}^{\mathrm{II}, k}(t)\right)$ are the integrals of the associated interactive forcing functions $\hat{G}^{\mathrm{I}, k}(t)$ and $\hat{G}^{\mathrm{I}, k-1}(t)$ (or $\hat{G}^{\mathrm{II}, k+1}(t)$ and $\left.\hat{G}^{\mathrm{II}, k}(t)\right)$ over $\left(t_{0}, t_{1}\right]$. Consider, for instance, the following two integrals in Equations (14a) and (14b) for $k=1$ :

$$
\begin{gathered}
\hat{I}^{\mathrm{I}, 1}=\int_{t_{0}}^{t_{1}} \exp \left[-A^{\mathrm{I}}\left(t-t_{0}\right)\right] \hat{G}^{\mathrm{I}, 0} \mathrm{~d} s \in \mathscr{R}^{2 n_{1}} \\
\hat{I}^{\mathrm{II}, 1}=\int_{t_{0}}^{t_{1}} \exp \left[-A^{\mathrm{II}}\left(t-t_{0}\right)\right] \hat{G}^{\mathrm{II}, 1} \mathrm{~d} s \in \mathscr{R}^{2 n_{2}}
\end{gathered}
$$

Noting that the elements of the integrand vectors are all bounded and the integration is over a small time interval $\left(t_{0}, t_{1}\right]$ (i.e. $\left.h_{1} \ll 1\right)$, one concludes that the (Euclidean) norm of the constant vector $\hat{I}^{\mathrm{I}, 1}$ (measuring the distance between $\hat{I}^{\mathrm{I}, 1}$ and the zero vector of the same dimension) can be bounded by

$$
\left\|\hat{I}^{\mathrm{I}, 1}\right\|=\left\{\sum_{j=1}^{2 n_{1}}\left(\hat{I}_{j}^{\mathrm{I}, 1}\right)^{2}\right\}^{1 / 2} \leqslant C^{\mathrm{I}, 1} h_{1}
$$

where $C^{\mathrm{I}, 1} \in \mathscr{R}^{+}$is a sufficiently large positive real number (independent of $h_{1}$ ). This means that the contribution of the integral containing $\hat{G}^{\mathrm{I}, 0}$ to $\hat{X}^{\mathrm{I}, 1}\left(t_{1}\right)$ is of order $O\left(h_{1}\right)$. Thus, one expects an error of order $O\left(h_{1}^{2}\right)$ in the approximation $\hat{X}^{\mathrm{I}, 1}\left(t_{1}\right)$ to $\hat{X}^{\mathrm{I}}\left(t_{1}\right)$ owing to the replacement of $\hat{G}^{\mathrm{I}}$ by $\hat{G}^{\mathrm{I}, 0}$. This can be checked by using the error term $E^{\mathrm{I}, 0}(t)$, given by (7a) and (7b), in an exact expression for the solution of $\hat{X}^{\mathrm{I}}(t)$ over $\left(t_{0}, t_{1}\right]$

$$
\begin{aligned}
\hat{X}^{\mathrm{I}}\left(t \mid t \in\left(t_{0}, t_{1}\right]\right)= & \exp \left[A^{\mathrm{I}}\left(t-t_{0}\right)\right] \hat{X}_{0}^{\mathrm{I}}+\exp \left[A^{\mathrm{I}}\left(t-t_{0}\right)\right] \\
& \times \int_{t_{0}}^{t} \exp \left[-A^{\mathrm{I}}\left(s-t_{0}\right)\right]\left\{\hat{F}^{\mathrm{I}}(s)+\hat{G}^{\mathrm{I}}\left(\hat{X}^{\mathrm{II}}(s)\right)\right\} \mathrm{d} s
\end{aligned}
$$

A comparison of the above equation with Equation (9a) reveals

$$
\hat{X}^{\mathrm{I}}(t)=\hat{X}^{\mathrm{I}, 1}(t)+\hat{J}^{\mathrm{I}, 1}(t), \quad t \in\left(t_{0}, t_{1}\right]
$$


where the error vector $\hat{J}^{\mathrm{I}, 1}(t)$ is given by

$$
\hat{J}^{\mathrm{I}, 1}(t)=\exp \left[A^{\mathrm{I}}\left(t-t_{0}\right)\right] \int_{t_{0}}^{t} \exp \left[-A^{\mathrm{I}}\left(s-t_{0}\right)\right]\left\{\hat{E}^{\mathrm{I}, 0}(s)\right\} \mathrm{d} s
$$

Here $\hat{E}^{\mathrm{I}, 0}(t)=\left(0^{\mathrm{T}},\left(E^{\mathrm{I}, 0}\right)^{\mathrm{T}}\right)^{\mathrm{T}} \in \mathscr{R}^{2 n_{1}}$ is the augmented error function. Since $\hat{E}^{\mathrm{I}, 0}(t)=O\left(t-t_{0}\right)$, we readily have

$$
\hat{J}^{\mathrm{I}, 1}(t)=O\left(t-t_{0}\right)^{2}
$$

and, thus, $\hat{J}^{\mathrm{I}, 1}\left(t_{1}\right)=O\left(h_{1}^{2}\right)$. It is noted that $\hat{G}^{\mathrm{II}}$ is a linear function of its argument $\hat{X}^{\mathrm{I}}(t)$ and hence may be decomposed (using Equations (18a) and (19)) as

$$
\hat{G}^{\mathrm{I}}(t)=\hat{G}^{\mathrm{II}}\left(\hat{X}^{\mathrm{I}}(t)\right)=\hat{G}^{\mathrm{II}}\left(\hat{X}^{\mathrm{I}, 1}+\hat{J}^{\mathrm{I}, 1}\right)=\hat{G}^{\mathrm{II}, 1}+O\left(t-t_{0}\right)^{2}
$$

for $t \in\left(t_{0}, t_{1}\right]$. Now, similar to Equation (17), the solution to the substructure $S^{\mathrm{II}}$ over the same interval is written as

$$
\begin{aligned}
\hat{X}^{\mathrm{II}}\left(t \mid t \in\left(t_{0}, t_{1}\right]\right)= & \exp \left[A^{\mathrm{II}}\left(t-t_{0}\right)\right] \hat{X}_{0}^{\mathrm{II}}+\exp \left[A^{\mathrm{II}}\left(t-t_{0}\right)\right] \\
& \times \int_{t_{0}}^{t} \exp \left[-A^{\mathrm{II}}\left(s-t_{0}\right)\right]\left\{\hat{F}^{\mathrm{II}}(s)+\hat{G}^{\mathrm{II}}\left(\hat{X}^{\mathrm{I}}(s)\right)\right\} \mathrm{d} s
\end{aligned}
$$

We thus have

$$
\hat{X}^{\mathrm{II}}(t)=\hat{X}^{\mathrm{II}, 1}(t)+\hat{J}^{\mathrm{II}, 1}(t), \quad t \in\left(t_{0}, t_{1}\right]
$$

where

$$
\hat{J}^{\mathrm{II}, 1}(t)=O\left(t-t_{0}\right)^{3}
$$

Following a similar logic, one may arrive at the following error orders for the $k$ th iterative step:

$$
\hat{J}^{\mathrm{I}, k}(t)=O\left(t-t_{0}\right)^{2 k}, \quad \hat{J}^{\mathrm{II}, k}(t)=O\left(t-t_{0}\right)^{2 k+1}, \quad k \in Z^{+}
$$

It then follows that $\hat{X}^{\mathrm{I}, k}(t)$ and $\hat{X}^{\mathrm{II}, k}(t)$, respectively, converge to the desired solutions $\hat{X}^{\mathrm{I}}(t)$ and $\hat{X}^{\mathrm{II}}(t)$ subject to the initial condition vector $\hat{X}_{0}^{\mathrm{I}}$ and $\hat{X}_{0}^{\mathrm{II}}$.

At the initial stages of sub-structural integration, no knowledge of the response of $S^{\mathrm{II}}$ is available (except for the initial conditions) while integrating the equations of motion for $S^{\mathrm{I}}$ and vice versa. Thus the iterative procedure, just outlined, is useful to obtain accurate estimates of the response. However, as the integration continues in time, information on the response accumulates. A natural question to ask is whether we can use this information to do away with the iterative process. Thus we intend to devise a non-iterative scheme, wherein the available information on the response of substructures at previous time instants is interpolated and/or extrapolated, while not significantly sacrificing the desired accuracy. This non-iterative process is briefly outlined next.

Let the targeted formal order of local accuracy be denoted by the positive integer $p_{l} \in Z^{+}$. This means that, if $\hat{X}(t)=\left(X(t)^{\mathrm{T}}, \dot{X}(t)^{\mathrm{T}}\right)^{\mathrm{T}} \in \mathscr{R}^{2 n}$ denotes the 'true' solution to the ordinary differential equations (ODEs) (4) and $\hat{X}^{\mathrm{R}, p_{l}}(t)=\left(\left(\hat{X}^{\mathrm{I}, p_{l}}(t)\right)^{\mathrm{T}},\left(\hat{X}^{\mathrm{II}, p_{l}}(t)\right)^{\mathrm{T}}\right)^{\mathrm{T}}$ denotes the RTS-based solution for any $t \in\left(t_{i-1}, t_{i}\right]$, then there is a real, positive constant $C$ such that

$$
\left\|\left(\hat{E}(t) \mid t \in\left(t_{i-1}, t_{i}\right]\right) \mid=\right\|\left(\hat{X}(t)-\hat{X}^{\mathrm{R}, p_{l}}(t) \mid t \in\left(t_{i-1}, t_{i}\right]\right) \| \leqslant C h_{i}^{p_{l}+1}
$$


Here $\|\hat{E}(t)\|$ is the Euclidean distance measure between the 'true' and RTS-based response vectors at any instant of time $t \in\left(t_{i-1}, t_{i}\right]$. The above inequality is only locally valid over $\left(t_{i-1}, t_{i}\right]$ with $\hat{X}(t)$ and $\hat{X}^{\mathrm{R}, p_{l}}(t)$ evolving with the same initial conditions, i.e. $\hat{X}\left(t_{i-1}\right)=\hat{X}^{\mathrm{R}, p_{l}}\left(t_{i-1}\right)=\hat{X}_{i-1}$. However, over the entire time interval of interest $\left(t_{0}, T\right]$ with $\hat{X}\left(t_{0}\right)=\hat{X}^{\mathrm{R}}\left(t_{0}\right)$, the order of the global error norm is given by

$$
\left\|\left(\hat{E}(t) \mid t \in\left(t_{0}, T\right]\right)\right\|=\left\|\left(\hat{X}(t)-\hat{X}^{\mathrm{R}}(t) \mid t \in\left(t_{0}, T\right]\right)\right\| \leqslant C h_{m}^{p_{l}}
$$

$\hat{X}^{\mathrm{R}}(t)$ is the RTS-based global response obtained by piecing together the local solutions

$$
\hat{X}^{\mathrm{R}}(t)=\sum_{i=1}^{N} \hat{X}^{\mathrm{R}, p_{l}}\left(t_{i}\right)\left\{H\left[t-t_{i-1}\right)-H\left(t-t_{i}\right)\right\}
$$

and $h_{m}=\max \left\{h_{i} \mid i=1,2, \ldots, N\right\}$. The unit step function $H\left(t-t_{i-1}\right)$ is defined as

$$
\begin{aligned}
H\left(t-t_{i}\right) & =1 & & \text { if } t>t_{i} \\
& =0 & & \text { otherwise }
\end{aligned}
$$

For further progress on the non-iterative sub-structural solution procedure, it is required that solutions at $p_{l}$ previous time instants are known. Thus consider the instant $t_{k}, k \geqslant p_{l}$, such that solutions at $p_{l}$ previous instants $t_{j}, j=k-p_{l}, \ldots, k-1$, have already been found. Now, starting with $S^{\mathrm{I}}$, an interpolating expansion for the interacting forcing vector $G^{\mathrm{I}}$ may be written as

$$
G^{\mathrm{I}} \cong G^{\mathrm{I}, p_{l}}(t)=\sum_{j=k-p_{l}}^{k-1} G_{j}^{\mathrm{I}} \Phi_{j}\left(t-t_{j}\right)
$$

where $G_{j}^{\mathrm{I}} \triangleq G^{\mathrm{I}}\left(\hat{X}^{\mathrm{II}}\left(t_{j}\right)\right)$ and $\left\{\Phi_{j}\left(t-t_{j}\right) \mid j=k-p_{l}, \ldots, k-1\right\}$ is a set of (interpolating) basis functions with $\Phi_{j}$ being centred at $t_{j}$, and $G^{\mathrm{I}, p_{l}}$ is an interpolating approximation to $G^{\mathrm{I}}$ using the series expansion (27) with $p_{l}$ terms. It is presently assumed that the response of the substructure $S^{\mathrm{II}}$ is uniformly continuous and sufficiently differentiable for any $t \in\left(t_{k-p_{l}}, t_{k}\right]$ so that the sequence of approximations $\left\{G^{\mathrm{I}, p_{l}}(t) \mid p_{l} \geqslant 2\right\}$ uniformly converges to $G^{\mathrm{I}}$ within $\left(t_{k-p_{l}}, t_{k}\right]$. An obvious choice of the basis functions $\Phi_{j}\left(t-t_{j}\right)$ is the set of interpolating Lagrangian polynomials (ILP-s) given by

$$
\Phi_{j}\left(t-t_{j}\right)=\prod_{\substack{i=k-p_{l} \\ i \neq j}}^{k-1} \frac{\left(t-t_{i}\right)}{\left(t_{j}-t_{i}\right)}
$$

It is noted that $\Phi_{j}$ is a polynomial (in $t$ ) of order $p_{l}-1$ and hence we expect to be able to approximate $G^{\mathrm{I}}$ with a (local) error $O\left(h_{m}^{p_{l}}\right)$, where $h_{m}=\max \left\{h_{i} \mid i \in\left[k-p_{l}, k-1\right]\right\}$. Moreover, this expansion remains valid to the same order for any $t \in\left(t_{k-p_{l}}, t_{k}\right]$ provided that $h_{k} \leqslant h_{m}$. This suggests a possible extrapolation of the approximation $G^{\mathrm{I}, p_{l}}(t)$ for $t_{k-1}<t \leqslant t_{k}$ with a local accuracy of order $O\left(h_{m}^{p_{l}-1}\right)$.

Before proceeding with the rest of the non-iterative scheme, we note that more informed choices of the basis function $\Phi_{j}$ are possible depending on the nature of the response of the associated dynamical system. For dynamical response with strong local characteristics (for instance, response under impact loads), interpolating wavelet functions or interpolets [41] could be used. 
In case we intend to obtain the RTS-based solution with a very high order of accuracy (i.e. $p_{l}$ is chosen to be quite high), use of higher order ILP-s may lead to spurious oscillations and a more appropriate choice for $\Phi_{j}$ could be in the form of distributed approximating functionals [42]. These considerations become particularly important in non-linear dynamics and will be discussed further in Section 5.

With the approximation of the interactive force given by Equation (27), the RTS-based solution of the substructure $S^{\mathrm{I}}$ for any $t \in\left(t_{k-1}, t_{k}\right]$ is given by

$$
\begin{aligned}
\hat{X}^{\mathrm{I}}\left(t \mid t \in\left(t_{k-1}, t_{k}\right]\right)= & \exp \left[A^{\mathrm{I}}\left(t-t_{k-1}\right)\right] \hat{X}_{k-1}^{\mathrm{I}}+\exp \left[A^{\mathrm{I}}\left(t-t_{k-1}\right)\right] \\
& \times \int_{t_{k-1}}^{t} \exp \left[-A^{\mathrm{I}}\left(s-t_{k-1}\right)\right]\left\{\hat{F}^{\mathrm{I}}(s)+\hat{G}^{\mathrm{I}, p_{l}}(s)+\hat{E}^{\mathrm{I}, p_{l}}(s)\right\} \mathrm{d} s
\end{aligned}
$$

where $\hat{E}^{\mathrm{I}, p_{l}}(t) \in \mathscr{R}^{2 n_{1}}$ is the augmented error function resulting from the replacement of the functional $G^{\mathrm{I}}\left(\hat{X}^{\mathrm{II}}(t)\right)$ by the function $\hat{G}^{\mathrm{I}, p_{l}}(t), p_{l} \geqslant 2$. If interest is only on local errors that accrue over $\left(t_{k-1}, t_{k}\right]$, one may condition $\hat{E}^{\mathrm{I}, p_{l}}(t)$ on the knowledge of $\hat{X}_{j}^{\mathrm{II}}, j=k-p_{l}, \ldots, k-1$, and the local initial condition $\hat{X}_{k-1}^{\mathrm{I}}$ leading to

$$
\left\|\left(\hat{E}^{\mathrm{I}, p_{l}}(t) \mid \hat{X}_{j}^{\mathrm{II}}, \hat{X}_{k-1}^{\mathrm{I}} ; j=k-p_{l}, \ldots, k-1\right)\right\| \leqslant C_{0}^{\mathrm{I}} h_{m}^{p_{l}}, \quad h_{k} \leqslant h_{m}
$$

The constant $C_{0}^{\mathrm{I}} \in \mathrm{R}^{+}$does not depend on $\hat{X}^{\mathrm{II}}$ and $h_{m}$. Using the above inequality in Equation (29) immediately leads to

$$
\left\|\left(\hat{X}^{\mathrm{I}}(t)-X^{\mathrm{I}, p_{l}}(t) \mid \hat{X}_{j}^{\mathrm{II}}, \hat{X}_{k-1}^{\mathrm{I}} ; j=k-p_{l}, \ldots, k-1\right)\right\| \leqslant K^{\mathrm{I}} h_{m}^{p_{l}+1}
$$

so that the local order of accuracy of the RTS-based solution of $S^{\mathrm{I}}$ is $O\left(h_{m}^{p_{l}}\right)$. Once the solution $\left(\hat{X}^{\mathrm{I}, p_{l}}(t) \mid t \in\left(t_{k-1}, t_{k}\right]\right)$ is known, we try obtaining $\hat{X}^{\mathrm{II}, p_{l}}(t)$ over the same time interval. Towards this, the interactive forcing vector $G^{\mathrm{II}, p_{l}}(t)$ is interpolated, based on the same scheme used in Equation (27), as

$$
G^{\mathrm{II}} \cong G^{\mathrm{II}, p_{l}}(t)=\sum_{j=k-p_{l}}^{k-1} G_{j}^{\mathrm{II}} \Phi_{j}\left(t-t_{j}\right)
$$

where $G_{j}^{\mathrm{II} \triangleq} G^{\mathrm{II}}\left(\hat{X}^{\mathrm{I}}\left(t_{j}\right) \mid j \in\left[k-p_{l}+1, k\right]\right)$. In particular, we note that the most recently available solution $\hat{X}_{k}^{\mathrm{I} \triangleq} \hat{X}^{\mathrm{I}}\left(t_{k}\right) \cong \hat{X}^{\mathrm{I}}, p_{l}\left(t_{k}\right)$ corresponding to $S^{\mathrm{I}}$ is exploited in the interpolating expansion (32). Use of this approximation for the interactive forcing immediately leads to the following solution for $S^{\mathrm{II}}$ :

$$
\begin{aligned}
\hat{X}^{\mathrm{II}, p_{l}}\left(t \mid t \in\left(t_{k-1}, t_{k}\right]\right)= & \exp \left[A^{\mathrm{II}}\left(t-t_{k-1}\right)\right] \hat{X}_{k-1}^{\mathrm{II}}+\exp \left[A^{\mathrm{II}}\left(t-t_{k-1}\right)\right] \\
& \times \int_{t_{k-1}}^{t} \exp \left[-A^{\mathrm{II}}\left(s-t_{k-1}\right)\right]\left\{\hat{F}^{\mathrm{II}}(s)+\hat{G}^{\mathrm{II}, p_{l}}(s)\right\} \mathrm{d} s
\end{aligned}
$$

Moreover, following the same reasoning as used in the case of $S^{\mathrm{I}}$, we may readily derive the following bound on the norm of the local error vector function:

$$
\left\|\left(\hat{E}^{\mathrm{II}, p_{l}}(t) \mid \hat{X}_{j}^{\mathrm{I}}, \hat{X}_{k-1}^{\mathrm{II}} ; j=k-p_{l}+1, \ldots, k\right)\right\| \leqslant C_{0}^{\mathrm{II}} h_{m}^{p_{l}}, \quad h_{k} \leqslant h_{m}
$$


Thus, we finally have

$$
\left\|\left(\hat{X}^{\mathrm{II}}(t)-X^{\mathrm{II}, p_{l}}(t) \mid \hat{X}_{j}^{\mathrm{I}}, \hat{X}_{k-1}^{\mathrm{II}} ; j=k-p_{l}+1, \ldots, k\right)\right\| \leqslant K^{\mathrm{II}} h_{m}^{p_{l}+1}
$$

The above non-iterative procedure may now be applied over succeeding time intervals to obtain the RTS-based approximation to the solution of the entire dynamical system (1).

It is interesting to observe that the integration scheme used here for substructural simulations provides a uniformly continuous and differentiable approximation to the original solution. This is evidenced in Equation (25) and is unlike the discrete integration procedures, such as Newmark or Runge-Kutta methods. Such a continuous approximation appears to be useful in tackling time delays (numerical as well as hardware-based). For the case of RTS involving non-linear substructures the present approach admits extensions through a local linearization method, such as the locally and MTL methods [36-38,43]: these are discussed in Section 5.

In order to compare the RTS-based results with true solutions of the system equations (4), we note that these equations may be solved in the same state-space formulation as done for the substructures to a high order of accuracy. Thus considering the time interval $\left(t_{i-1}, t_{i}\right]$ with uniform step size $h=t_{i}-t_{i-1}$, the solution vector $\hat{X}(t)=\left(X^{\mathrm{T}}(t), \dot{X}^{\mathrm{T}}(t)\right)^{\mathrm{T}} \in \mathbf{R}^{2 n}$ may be written as

$$
\begin{aligned}
\hat{X}\left(t \mid t \in\left(t_{i-1}, t_{i}\right]\right)= & \exp \left[A\left(t-t_{i-1}\right)\right] \hat{X}_{i-1}+\exp \left[A\left(t-t_{i-1}\right)\right] \\
& \times \int_{t_{i-1}}^{t} \exp \left[-A\left(s-t_{i-1}\right)\right]\{\hat{F}(s)\} \mathrm{d} s
\end{aligned}
$$

The system coefficient matrix is given by

$$
A=\left[\begin{array}{cc}
0 & I \\
-[M]^{-1} K & -[M]^{-1} C
\end{array}\right] \in \mathscr{R}^{2 n \times 2 n}
$$

Supposing that a solution of local order $O\left(h^{4}\right)$ is needed, the matrix exponents of the system coefficient matrix may be written as

$$
\begin{aligned}
\exp (A h) & \cong I+A h+0.5 A^{2} h^{2}+\frac{1}{6} A^{3} h^{3}+\frac{1}{24} A^{4} h^{4} \\
\exp \left(-A h_{s}\right) & \cong I-A h_{s}+0.5 A^{2} h_{s}^{2}-\frac{1}{6} A^{3} h_{s}^{3}
\end{aligned}
$$

where $h_{s}=s-t_{i-1}, t_{i-1}<s \leqslant t_{i}$ and $I$ is the identity matrix of an appropriate order. It is noted that $\exp \left(-A h_{s}\right)$ is expanded to one order less than $\exp (-A h)$. This is possible as $\exp \left(-A h_{s}\right)$ is a part of an integrand where integration is performed over a step size $h$. In other words, if the integrand is of order $O\left(h_{s}^{3}\right)$, then the integral must be of order $O\left(h^{4}\right)$. Moreover, in case integration over the entire interval uses a uniform $h$, then we have

$$
\exp \left(A\left(t_{i+n}-t_{i}\right)\right)=\exp (A n h)=[\exp (A h)]^{n}
$$

Thus, we need to compute $\exp (A h)$ only once using (36c) and then use matrix multiplications to generate the required exponentials for succeeding time intervals. 


\section{RTS WITH COMBINED NUMERICAL AND TEST MODELS}

The proposed RTS formulation is based on treating both the substructures, $S^{\mathrm{I}}$ and $S^{\mathrm{II}}$, as numerical substructures. However, this need not be the case. For instance, one could treat $S^{\mathrm{I}}$ as the numerical substructure and $S^{\mathrm{II}}$ as the test substructure, whose response is measured via an experiment conducted in the laboratory. During the development of the RTS methodology, it has been observed that the solution strategy of one of the substructures does not require any information on the system parameters (i.e. mass, damping and stiffness) of the other substructure. This is made possible by the introduction of the interactive forces that are conditional functions of time only. In other words, the substructures correspond dynamically amongst themselves only through the interactive forces. Thus, let $S^{\mathrm{I}}$ and $S^{\mathrm{II}}$ be numerical and test substructures, respectively. We may start with $S^{\mathrm{I}}$, the numerical substructure. To begin with, only a crude estimate of the interactive forces on $S^{\mathrm{I}}$ is available via the known initial conditions of $S^{\mathrm{II}}$. Thus, given a formal order of accuracy $p_{l}$, the sub-structural responses of $S^{\mathrm{I}}$ and $S^{\mathrm{II}}$ need to be found iteratively and improved at least to order $O\left(h_{m}^{p_{l}}\right)$ over the initial $p_{l}$ time steps. It is only at this stage that the non-iterative procedure may be applied.

It is assumed that the test substructure is dynamically excited at the points of interaction with the numerical substructure through suitable actuation devices. Moreover, these points are taken to be adequately equipped with transducers to measure reactions that serve as forcing functions to the numerical substructure. Satisfaction of the basic purpose of RTS requires that numerical calculations on $S^{\mathrm{I}}$ as well as experimental actuations and measurements on $S^{\mathrm{II}}$ proceed in real time (i.e. in the same time scale in which the entire structure, consisting of $S^{\mathrm{I}}$ and $S^{\mathrm{II}}$ is vibrating). Adherence to this requirement becomes particularly crucial for a wide class of materially non-linear response regimes wherein the structural resistance to velocity and displacement at any time instant becomes a function of response time history up to that instant. Unfortunately, the iterative method of improving the response accuracy during start-up is quite time consuming, especially for cases where the dimension of $S^{\mathrm{I}}$ is quite high and hence it cannot generally be applied in real time. However this may not be a major limitation for most cases of interest, wherein history-dependent material non-linearity (often associated with material degradation and damage) takes some time to set in. This generally should allow enough time before one is ready to apply the non-iterative procedure in real time.

In order to frame a precise set of steps for a practical implementation of the RTS procedure for combined numerical and test substructures, it is useful to take a brief look at some sources of possible delays and account for these delays, if possible. In Figure 2, typical delay times over $\left(t_{i-1}, t_{i}\right]$ are summarized. One must choose $h_{i}=t_{i}-t_{i-1}$ such that it is at least equal to the sum of all the delay times (i.e. $h_{i} \geqslant \Delta t_{1}+\Delta t_{2}+\Delta t_{3}$ ). In the following, the major steps involved in implementing the RTS-based simulation with one numerical substructure $S^{\mathrm{I}}$ and another test substructure $S^{\mathrm{II}}$ are described.

\section{Phase I: Iterative computations}

Step 1: Choose $p_{l}$. Choose an appropriate value of $T_{\mathrm{I}}>t_{0}$ such that iterative computations are completed during $\left(t_{0}, T_{\mathrm{I}}\right]$. This part of calculations essentially ensures that the effect of initial conditions is correctly captured. In the context of materially non-linear dynamical systems, it is assumed that material degradation does not occur for $t \leqslant T_{\mathrm{I}}$. This is generally considered acceptable since the response is unlikely to cross limits of linear behaviour within the short time interval 


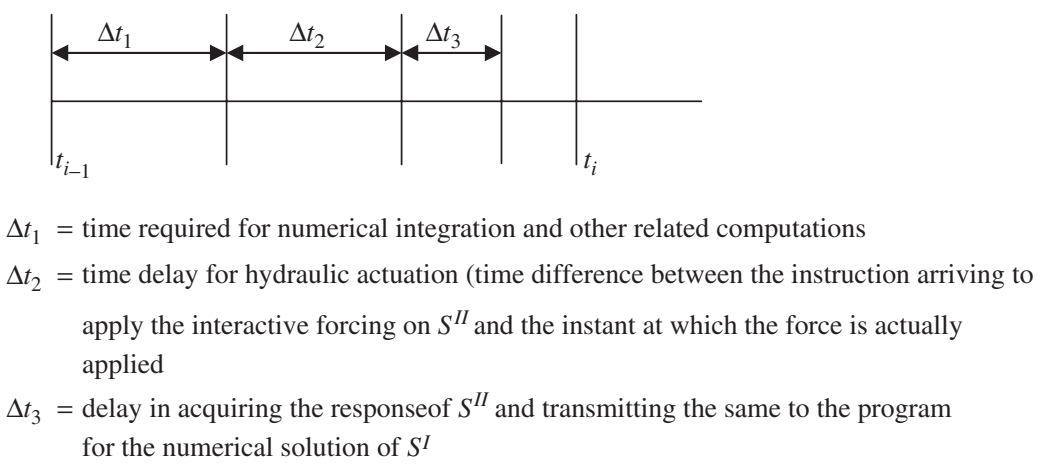

Figure 2. A schematic representation of a few typical delays: $h_{i}=t_{i}-t_{i-1} \geqslant \sum_{j=1}^{3} \Delta t_{j}$.

of $T_{\mathrm{I}}$. Divide the interval $\left(t_{0}, T_{\mathrm{I}}\right]$ into $p_{l}$ subdivisions using grid points $\left\{t_{0}, t_{1}, \ldots, t_{p_{l}}=T_{\mathrm{I}}\right\}$ such that the maximum step size does not exceed $h_{m}$.

Step 2: Apply the iterative technique over $\left(t_{0}, T_{\mathrm{I}}\right.$ ] in a non-real-time setting. Consider, for instance, the $i$ th interval $\left(t_{i-1}, t_{i}\right], 1 \leqslant i \leqslant p_{l}$ and the $j$ th iterative solution. Start with $S^{\mathrm{I}}$, the numerical substructure. Find $\hat{G}^{\mathrm{I}, j-1}(t)$ and obtain the response $\hat{X}^{\mathrm{I}, j}(t)$.

Step 3: Based on $\hat{X}^{\mathrm{I}, j}(t)$, obtain $\hat{G}^{\mathrm{II}, j}(t)$. Using the system of actuators apply the force $\hat{F}^{\mathrm{II}}(t)+\hat{G}^{\mathrm{II}, j}(t)$ on $S^{\mathrm{II}}$, the test substructure, while subjecting it to the known initial conditions $\hat{X}_{i-1}^{\mathrm{II}} \triangleq \hat{X}^{\mathrm{II}}\left(t_{i-1}\right)$. Measure interaction forces at the interface dofs via an appropriately installed transduction and data acquisition system.

Step 4: Go back to steps 3, 4 and obtain the response $\hat{X}^{\mathrm{I}, j}(t)$ for increasing $j$ till $\left\langle\| \hat{X}^{\mathrm{R}, j+1}-\right.$ $\left.\hat{X}^{\mathrm{R}, j} \|\right\rangle \leqslant O\left(h_{m}^{p_{l}+1}\right)$ where $h_{m}=\max _{i \in\left[1, p_{l}\right]}\left\{h_{i}=t_{i}-t_{i-1}\right\}$.

Step 5: Repeat the above steps for all $i \in\left[1, p_{l}\right]$.

\section{Phase II: Non-iterative computations in real time}

Step 6: Consider the interval $\left(t_{i-1}, t_{i}\right]$ for $i \geqslant p_{l}+1$ and start with $S^{\mathrm{I}}$. Note that $h_{i}$ must be so chosen as to satisfy $h_{i}=t_{i}-t_{i-1} \geqslant \Delta t_{1}+\Delta t_{2}+\Delta t_{3}$. Use an interpolation over the grid points $\left\{t_{i-p_{l}}, \ldots, t_{i-1}\right\}$ to construct $\hat{G}^{\mathrm{I}, p_{l}}(t)$ and extrapolate the same function over $\left(t_{i-1}, t_{i}\right]$. Obtain the response $\left(\hat{X}^{\mathrm{I}, p_{l}}(t) \mid t \in\left(t_{i-1}, t_{i}\right]\right)$ under the force $\hat{F}^{\mathrm{I}}(t)+\hat{G}^{\mathrm{I}, p_{l}}(t)$.

Step 7: Based on $\hat{X}^{\mathrm{I}, p_{l}}(t)$, compute $\hat{G}^{\mathrm{II}, p_{l}}\left(t_{i}\right)$. Send a signal to the actuating devices for $S^{\mathrm{II}}$ to apply a force vector $\hat{F}^{\mathrm{II}}\left(t_{i}\right)+\hat{G}^{\mathrm{II}, p_{l}}\left(t_{i}\right)$ at $t=t_{i-1}+\Delta t_{1}$.

Step 8: Acquire and store the response signal $\hat{X}^{\mathrm{II}, p_{l}}\left(t_{i}\right)$ within a sufficiently small time interval such that the time $t$ at the completion of this process remains less than $t_{i}$. In case one has stored the computer program for the numerical analysis of $S^{\mathrm{I}}$ on a DSP chip and communicating with $S^{\mathrm{II}}$ through a real-time operating system (RTOS), the acquired data on interaction forces should ideally also be stored on the DSP chip.

Step 9: Wait till $t=t_{i}$ and start the next numerical integration for $S^{\mathrm{I}}$ precisely at $t=t_{i}$.

Step 10: Repeat steps 6 through 9 for increasing $i$ till the RTS-based simulation is completed over the entire time interval $\left(t_{0}, T\right]$ of interest.

Finally, we note that the above procedure admits a ready generalization for RTS systems with arbitrary numbers and arrangements of numerical and test substructures. 


\section{SPECTRAL CHARACTERISTICS AND STABILITY OF NUMERICAL SOLUTIONS}

While a formal order of accuracy for the non-iterative part of the numerical solution has already been established, local errors may still grow in time depending on the spectral characteristics of the matrix, $B_{p_{l}}(h) \in \mathscr{R}^{2 n_{1} \times 2 n_{1}}$, defining the homogeneous mapping

$$
\hat{X}_{k+1}^{\mathrm{I}, p_{l}}=B_{p_{l}}(h) \hat{X}_{k}^{\mathrm{I}}
$$

Here $\hat{X}_{k+1}^{\mathrm{I}, p_{l}} \triangleq\left(\left(X^{\mathrm{I}, p_{l}}\left(t_{k+1}\right)\right)^{\mathrm{T}},\left(\dot{X}^{\mathrm{I}, p_{l}}\left(t_{k+1}\right)\right)^{\mathrm{T}}\right)^{\mathrm{T}} \in \mathscr{R}^{2 n_{1}}$ denotes the approximate solution of the numerical substructure at $t=t_{k+1}$ subject to the local initial condition vector $\hat{X}_{k}^{\mathrm{I}}$ and $h=t_{k+1}-t_{k}$ is the uniform step size. Recall that $B_{p_{l}}(h)$ is obtained by truncated Taylor expansion of $\exp \left(A^{\mathrm{I}} h\right)$ up to and including $O\left(h^{p_{l}}\right)$

$$
B_{p_{l}}(h)=I+A^{\mathrm{I}} h+\cdots+\left[A^{\mathrm{I}}\right]^{p_{l}} \frac{h^{p_{l}}}{p_{l} !}
$$

Equation (38) is an $O\left(h^{p_{l}}\right)$ solution for the following homogeneous ODEs at $t=t_{k+1}$

$$
\dot{\hat{X}}^{\mathrm{I}}(t)=A^{\mathrm{I}} \hat{X}^{\mathrm{I}}(t)
$$

subject to the initial condition $\hat{X}^{\mathrm{I}}\left(t_{k}\right)=\hat{X}_{k}^{\mathrm{I}}$. The true solution at $t=t_{k+1}$ is

$$
\hat{X}_{k+1}^{\mathrm{I}}=B(h) \hat{X}_{k}^{\mathrm{I}}, \quad B(h)=\exp \left(A^{\mathrm{I}} h\right)
$$

Let $P \in \mathscr{R}^{2 n_{1} \times 2 n_{1}}$ be the unique, invertible matrix used to transform $A^{\mathrm{I}}$ to its Jordan form

$$
P^{-1} A^{\mathrm{I}} P=J^{\mathrm{I}}
$$

where $J^{\mathrm{I}}$ is the Jordan's form for the numerical substructure I with eigenvalues $\lambda_{1}, \ldots, \lambda_{2 n_{1}}$, some of which are probably repeated. Since one has

$$
P^{-1}\left[A^{\mathrm{I}}\right]^{m} P=\left[J^{\mathrm{I}}\right]^{m}, \quad m=1,2, \ldots
$$

$\left[J^{\mathrm{I}}\right]^{m}$ (i.e. the $m$ th power of $J^{\mathrm{I}}$ ) has eigenvalues $\lambda_{1}^{m}, \ldots, \lambda_{2 n_{1}}^{m}$. Thus, we have

$$
P^{-1} B(h) P=P^{-1} \exp \left(A^{\mathrm{I}} h\right) P=P^{-1}\left[\sum_{m=0}^{\infty}\left[A^{\mathrm{I}}\right]^{m} \frac{h^{m}}{m !}\right] P=\sum_{m=0}^{\infty}\left[J^{\mathrm{I}}\right]^{m} \frac{h^{m}}{m !}
$$

So, if $\gamma_{1}, \ldots, \gamma_{2 n_{1}}$ denote $2 n_{1}$ eigenvalues of $B(h)$ and $\bar{\gamma}_{1}, \ldots, \bar{\gamma}_{2 n_{1}}$ are the corresponding eigenvalues of $B_{p_{l}}(h)$, then the following relations hold:

$$
\gamma_{j}=\bar{\gamma}_{j}+O\left(h^{p_{l}+1}\right), \quad j=1, \ldots, 2 n_{1}
$$

In other words, the state-space formulation preserves the eigenvalues modulo $O\left(h^{p_{l}}\right)$. However, in linear time-invariant structural dynamics, while contributions to the response from the higher order modes are small, their inclusion may lead to noise in the numerical solution [1]. In fact, if $p_{l}$ is chosen high during numerical integrations using Equation (38), then appreciable floating point errors may accrue and, worse still, these errors may interact with contributions from higher order modes and grow in time. 
In order to enable the scheme to 'dissipate' such a growth, the following implicit form of the explicit map, given by Equation (38), is introduced:

$$
\hat{X}_{k+1}^{\mathrm{I}, p_{l}}=\left(I+A^{\mathrm{I}} h+\cdots+(1-\alpha)\left[A^{\mathrm{I}}\right]^{p_{l}} \frac{h^{p_{l}}}{p_{l} !}\right) \hat{X}_{k}^{\mathrm{I}}+\alpha\left[A^{\mathrm{I}}\right]^{p_{l}} \frac{h^{p_{l}}}{p_{l} !} \hat{X}_{k+1}^{\mathrm{I}, p_{l}}
$$

where $\alpha$ is a real parameter. Since one may write

$$
\hat{X}_{k+1}^{\mathrm{I}, p_{l}}=\hat{X}_{k}^{\mathrm{I}}+\left.\frac{\mathrm{d}}{\mathrm{d} t} \hat{X}^{\mathrm{I}}(t)\right|_{t=t_{k}} h+O\left(h^{2}\right)
$$

substitution of Equation (46) into (45) and a subsequent comparison with (38) and (39) show that the formal orders of accuracy via Equations (38) and (45) are the same up to $O\left(h^{p_{l}}\right)$ terms. Thus, the formal order of accuracy remains unaffected if the implicit scheme (45) is used. Equation (45) may be recast as

$$
\left[I-\alpha\left[A^{\mathrm{I}}\right]^{p_{l}} \frac{h^{p_{l}}}{p_{l} !}\right] \hat{X}_{k+1}^{\mathrm{I}, p_{l}}=\left[I+A^{\mathrm{I}} h+\cdots+(1-\alpha)\left[A^{\mathrm{I}}\right]^{p_{l}} \frac{h^{p_{l}}}{p_{l} !}\right] \hat{X}_{k}^{\mathrm{I}}
$$

or

$$
\hat{X}_{k+1}^{\mathrm{I}, p_{l}}=\left[Q_{p_{l}}(h)\right] \hat{X}_{k}^{\mathrm{I}}
$$

Following the concept of spectral stability [44], it follows that the implicit time-stepping algorithm (47b) is stable if and only if

(a) the spectral radius of $\left[Q_{p_{l}}(h)\right]$, denoted herein as $\rho\left[Q_{p_{l}}(h)\right]$, is $\leqslant 1$;

(b) eigenvalues of modulus 1 are simple (i.e. their multiplicity is 1 ).

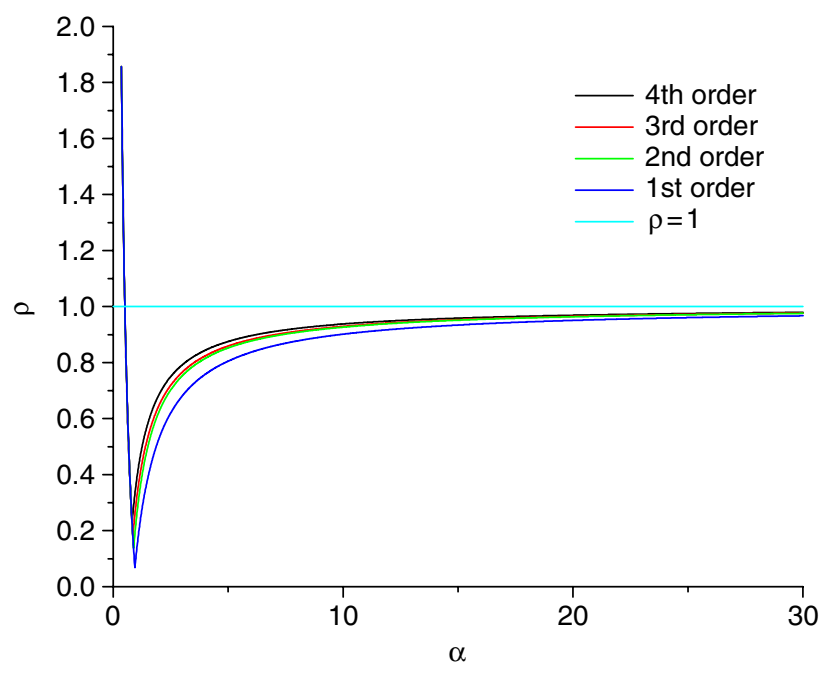

Figure 3. Spectral radius versus $\alpha$ via methods of different orders: $\omega^{2}=200$ (about $2.25 \mathrm{~Hz}$ ) and $\eta=20.0, h=0.01$. 

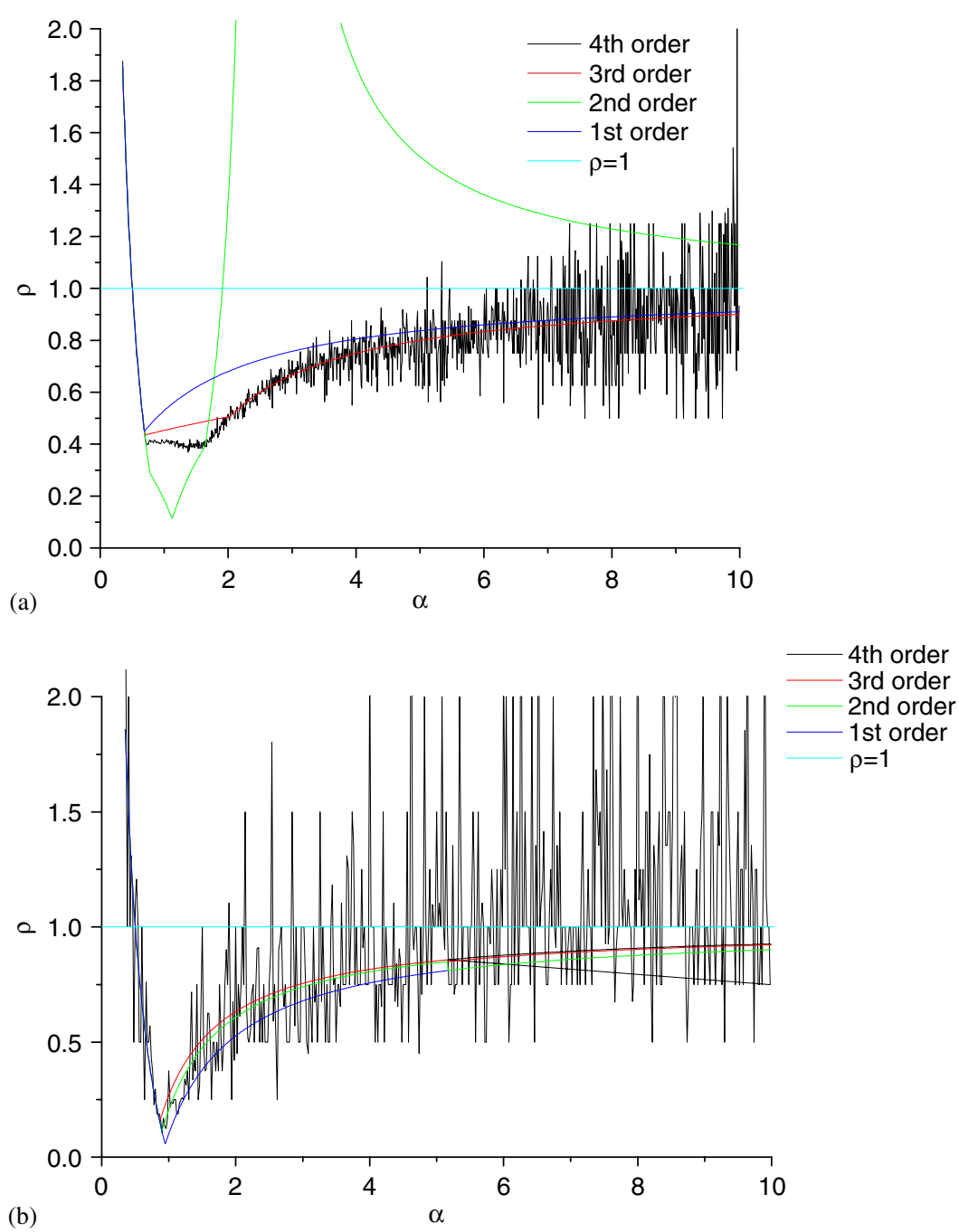

Figure 4. Spectral radius versus $\alpha$ via methods of different orders in the high-frequency range: (a) $\omega^{2}=35530.6$ (about $30.0 \mathrm{~Hz}$ ), $\eta=200.0, h=0.01$ and (b) $\omega^{2}=35530.6$ (about $\left.30.0 \mathrm{~Hz}\right), \eta=200.0, h=0.1$.

Recall that the spectral radius $\rho\left[Q_{p_{l}}(h)\right]$ is defined to be the maximum modulus of the eigenvalues of $Q_{p_{l}}(h)$. This definition of stability fails to hold, as the dimension of the dynamical system tends to become infinite $[45,46]$, i.e. when the system is modelled via partial differential equations. However, in order not to lose focus, attention is presently restricted to numerical stability of only a given, finite-dimensional semi-discrete system of equations of motion. In such cases, one may argue that the time-stepping method, as described above, is stable if and only if it is modally stable. In other words, the norm of the error in evaluating $\hat{X}^{\mathrm{I}}(t)$ for a given $t$ is bounded by the 


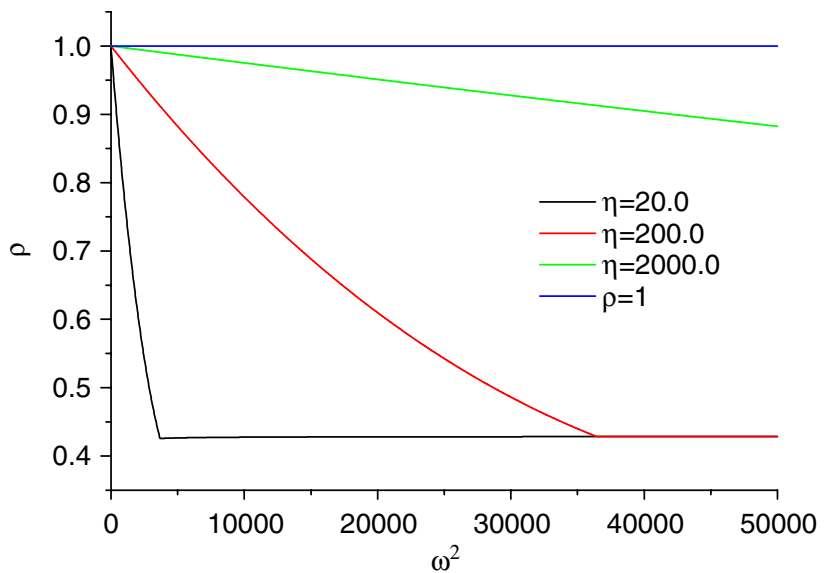

Figure 5. Spectral radius versus $\omega^{2}$ for different values of the modal damping parameter, $\eta$, using the third order method: $\alpha=0.7, h=0.01$.

sum (over all the modes) of the norms of errors in evaluating modal displacements and velocities. See Romero [45] for a formal proof of this statement. Thus, it suffices to consider the following modal equations of motion:

$$
\begin{aligned}
& \dot{x}=y \\
& \dot{y}=-2 \eta \omega y-\omega^{2} x
\end{aligned}
$$

and study the spectral stability of the associated matrix $Q_{p_{l}}(h) \in \mathscr{R}^{2 \times 2}$. The real quantities $\eta$ and $\omega$ are, respectively, the modal damping and frequency parameters.

In Figure 3, plots of the spectral radius $\rho$ as a function of the implicitness parameter $\alpha$ is provided for different orders of accuracy $p_{l}$. The modal frequency parameter $\omega$ is taken to be quite low, around $2.25 \mathrm{~Hz}$. In this case, all the four methods appear to have similar stability characteristics and all of them are unstable at and around $\alpha=0$. We note that $\alpha=0$ corresponds to the explicit scheme. However, the scenario changes drastically as $\omega$ increases. Such a case with $\omega \approx 30 \mathrm{~Hz}$ is reported in Figure 4(a) with a time step size $h=0.01$. While the first and third order methods still remain stable over almost the same range of $\alpha$ (as in Figure 3), the second order method shows instability for $\alpha>1.8$. Moreover, the stability curve for the fourth order method shows a noisy trend, which implies a propagation of numerical errors in time. In fact, as reported in Figure 4(b), the level of noise in the stability curve corresponding to the fourth order method conspicuously increases with increasing $h$. However, we have observed that the stability of the third order method is nearly independent of $h$. Figure 5 shows plots of $\rho$ versus $\omega^{2}$ for different choices of the damping parameter, $\eta$, and we see that the stability characteristics of the third order method are not compromised due to choice of $\omega^{2}$. Unlike the third order method, the fourth order method shows a uniformly noisy stability curve for different choices of $h$, especially in the high-frequency regime. In fact, the higher the order of the method beyond the third, the higher is the possibility of propagation of numerical errors. 


\section{RTS WITH NON-LINEAR SUBSTRUCTURES}

\subsection{The methodology}

The procedure proposed in Section 2, consisting of an initial iterative phase followed by a subsequent non-iterative phase, is extended to cover non-linear numerical and/or experimental substructures. In order to outline the procedure, let the given structure be made up of two non-linear substructures $S^{\mathrm{I}}$ and $S^{\mathrm{II}}$. One may express the equation of motion of $S^{\mathrm{I}}$, in a form similar to Equation (5), as

$$
M^{\mathrm{I}} \ddot{X}^{\mathrm{I}}+C^{\mathrm{I}} \dot{X}^{\mathrm{I}}+K^{\mathrm{I}} X^{\mathrm{I}}=F^{\mathrm{I}}(t)-G^{\mathrm{I}}\left(\ddot{X}^{\mathrm{II}}, \dot{X}^{\mathrm{II}}, X^{\mathrm{II}}\right)+Q^{\mathrm{I}}\left(X^{\mathrm{I}}, \dot{X}^{\mathrm{I}}\right)
$$

with initial conditions $\left[X_{0}^{\mathrm{I}} \dot{X}_{0}^{\mathrm{I}}\right]^{\mathrm{T}}$. Here the additional term $Q^{\mathrm{I}}\left(X^{\mathrm{I}}, \dot{X}^{\mathrm{I}}\right)$ is a non-linear vector function, resulting from the non-linearity in the stiffness and damping of $S^{\mathrm{I}}$ and all other terms are having the same meaning as recorded in Section 2. One can also write the equation of motion of $S^{\mathrm{II}}$ in a similar manner.

Now consider the first time step $\left(t_{0}, t_{1}\right]$. Equation (49) cannot be integrated explicitly due to lack of knowledge of the functions $G^{\mathrm{I}}$ and $Q^{\mathrm{I}}$ except at $t=t_{0}$, where they are given by $G^{\mathrm{I}, 0}=G^{\mathrm{I}}\left(\ddot{X}_{0}^{\mathrm{II}}, \dot{X}_{0}^{\mathrm{II}}, X_{0}^{\mathrm{II}}\right)$ and $Q^{\mathrm{I}, 0}=Q^{\mathrm{I}}\left(X_{0}^{\mathrm{I}}, \dot{X}_{0}^{\mathrm{I}}\right)$. Approximating $G^{\mathrm{I}}\left(\ddot{X}^{\mathrm{II}}, \dot{X}^{\mathrm{II}}, X^{\mathrm{II}}\right) \cong G^{\mathrm{I}, 0}$ and $Q^{\mathrm{I}}\left(X^{\mathrm{I}}, \dot{X}^{\mathrm{I}}\right) \cong Q^{\mathrm{I}, 0}$ as constant functions uniformly over the time interval $\left(t_{0}, t_{1}\right]$ would probably lead to unacceptably high numerical errors. Alternatively, an iterative method that uses a semi-analytical integration scheme based on the MTL method [36] is developed. For this purpose, we express Equation (49) in the state-space form as

$$
\dot{Y}^{\mathrm{I}}=A^{\mathrm{I}} Y^{\mathrm{I}}+U^{\mathrm{I}}
$$

where

$$
Y^{\mathrm{I}}=\left\{\begin{array}{c}
X^{\mathrm{I}} \\
\dot{X}^{\mathrm{I}}
\end{array}\right\} \in \Re^{2 n_{1}}, \quad A^{\mathrm{I}}=\left[\begin{array}{cc}
0 & I \\
-\left[M^{\mathrm{I}}\right]^{-1} K^{\mathrm{I}} & -\left[M^{\mathrm{I}}\right]^{-1} C^{\mathrm{I}}
\end{array}\right] \in \Re^{2 n_{1} \times 2 n_{1}}
$$

and

$$
U^{\mathrm{I}}=\left\{\begin{array}{c}
\{0\} \\
-\left[M^{\mathrm{I}}\right]^{-1}\left\{F^{\mathrm{I}}(t)-G^{\mathrm{I}}\left(\ddot{X}^{\mathrm{II}}, \dot{X}^{\mathrm{II}}, X^{\mathrm{II}}\right)+Q^{\mathrm{I}}\left(X^{\mathrm{I}}, \dot{X}^{\mathrm{I}}\right)\right\}
\end{array}\right\} \in \Re^{2 n_{1}}
$$

with $0, I \in \Re^{n_{1} \times n_{1}}$ being zero and identity matrices, respectively, and $\{0\} \in \Re^{n_{1}}$, the zero vector. Solution of Equation (50) for $t \in\left(t_{0}, t_{1}\right]$ may be written as

$$
Y^{\mathrm{I}}(t)=\mathrm{e}^{A^{\mathrm{I}}\left(t-t_{0}\right)} Y^{\mathrm{I}, 0}+\int_{t_{0}}^{t} \mathrm{e}^{A^{\mathrm{I}}(t-\tau)} U^{\mathrm{I}}(\tau) \mathrm{d} \tau
$$

where $Y^{\mathrm{I}, 0}=\left\{\begin{array}{c}X_{0}^{\mathrm{I}} \\ \dot{X}_{0}^{\mathrm{I}}\end{array}\right\}$. Let the time step $\left(t_{0}, t_{1}\right]$ be divided into $s$ smaller intervals as $\left(t^{0}, t^{1}, t^{2}, \ldots, t^{s}\right)$ where $t^{0}=t_{0}$ and $t^{s}=t_{1}$. To start the iteration, one can use the uniform approximation $G^{\mathrm{I}}\left(\ddot{X}^{\mathrm{II}}, \dot{X}^{\mathrm{II}}\right.$, $\left.X^{\mathrm{II}}\right) \cong G^{\mathrm{I}, 0}$ and $Q^{\mathrm{I}}\left(X^{\mathrm{I}}, \dot{X}^{\mathrm{I}}\right) \cong Q^{\mathrm{I}, 0}$ at all the grid points and thus integrate the equation of motion of 
$S^{\mathrm{I}}$ explicitly using Equation (51), without the knowledge of the response of the other substructure $S^{\mathrm{II}}$ over the same time interval. The approximate solution so obtained can be improved in the subsequent iterations in the same manner as explained in Section 2. This iterative procedure can be stopped when it satisfies the convergence criteria $\left\|Y^{\mathrm{I}, j}-Y^{\mathrm{I}, j-1}\right\|<\varepsilon$, where $j$ refers to the iteration number, $\|$.$\| denotes the Euclidean norm and \varepsilon$ is a specified tolerance. This iteration strategy can be applied during the succeeding time intervals $\left(t_{i-1}, t_{i}\right], \rightleftharpoons i \geqslant 2$ to obtain the response of $S^{\mathrm{I}}$ and $S^{\mathrm{II}}$. The iterative phase can be stopped when the information available is sufficient to start the non-iterative phase, wherein the available information of the response of the substructures at the previous time instances are suitably interpolated and/or extrapolated to carry out the integration process. In the non-iterative phase, the components of the interactive force and non-linear vector function need to be extrapolated to carry out the integration of the numerical substructure in real time. It is clear that the accuracy of the extrapolation depends on the choice of the basis functions $\Phi_{j}(t)$. One may use the ILP, given in Equation (28) as basis functions. However, in the context of non-linear substructures, we select $\Phi_{j}(t)$ based on the RKPM for reasons explained below.

In situations involving large time delays (e.g. an increase in the actuator time delay with an increase in the stiffness of the test substructure, Darby et al. [47]), one has to choose large time steps. This may seriously affect the performance of the RTS simulation, particularly for nonlinear systems. One way to circumvent this difficulty is to extrapolate the actuator displacement over multiple time steps ahead so that adequate time is available to achieve the required actuator displacement without affecting the accuracy of extrapolation. While the extrapolation using ILP yields satisfactory results for single-step extrapolations, it is found to be unsuitable over multiple steps. This underlines the need to develop a more accurate multi-step extrapolation technique. With this in mind, an extrapolation technique (hereafter referred to as the RK-based method) within the framework of the RKPM is developed next.

\subsection{Multi-step extrapolations using the reproducing kernel approximation}

The RKPM has been developed in the existing literature as a means to approximately solve partial differential equations of interest in engineering applications [48, 49]. This method has been originally proposed to overcome the difficulties of mesh distortion and mesh refinement, often experienced in the finite element analysis of high-frequency mechanical or structural systems and large deformation problems. The RKPM, popularly known as the mesh-free method, uses a set of nodes or particles in the problem domain rather than an element-based domain discretization as employed in the finite element method. Several versions of the RKPM are available in the literature [40, 50-53]. In the RKPM, the approximate solution is constructed by a reproducing kernel (RK) function; and in the present work, we exploit these functions to develop an extrapolation scheme in the context of RTS simulations. Before we consider the problem of extrapolation, we summarize first the RKPM-based procedure for interpolating a function.

Consider a one-dimensional domain $\Omega$ discretized by a set of points (particles), $\left\{t_{i}, t_{i} \in \Omega\right\}_{i=1}^{N_{p}}$. Let $u(t)$ be a sufficiently smooth function defined by the values $\left\{u_{i}=u\left(t_{i}\right)\right\}_{i=1}^{N_{p}}$ at these points. The function $u(t)$ is approximated as

$$
u^{a}(t)=\sum_{i=1}^{N_{p}} \Psi_{i}(t) u\left(t_{i}\right)
$$


where $\Psi_{i}(t),\left[i \in 1, N_{p}\right]$ are called the RK shape functions. The RK shape functions are constructed as

$$
\Psi_{i}(t)=c\left(t-t_{i}\right) \phi_{a_{i}}\left(t-t_{i}\right)
$$

where $c\left(t-t_{i}\right)$ is a correction function and $\phi_{a_{i}}\left(t-t_{i}\right)$ is a kernel or window function centred at $t_{i}$ and having a compact support. The parameter $a_{i}$ is the dilation parameter, which defines the support of the kernel function. Gaussian or cubic spline functions are commonly used as kernel functions. The correction function is typically expressed as a linear combination of polynomial basis functions as

$$
c\left(t-t_{i}\right)=b_{0}(t)+b_{1}(t)\left(t-t_{i}\right)+b_{2}(t)\left(t-t_{i}\right)^{2}+\cdots+b_{n}(t)\left(t-t_{i}\right)^{n}
$$

where $n$ is the highest degree of polynomials exactly reproduced by the RK approximation. Using the notations

$$
H\left(t-t_{i}\right)=\left\{1, t-t_{i},\left(t-t_{i}\right)^{2}, \ldots,\left(t-t_{i}\right)^{n}\right\}^{\mathrm{T}}
$$

and

$$
b(t)=\left\{b_{0}(t), b_{1}(t), \ldots, b_{n}(t)\right\}^{\mathrm{T}}
$$

Equation (54) may be recast as

$$
c\left(t-t_{i}\right)=H^{\mathrm{T}}\left(t-t_{i}\right) b(t)
$$

Furthermore, Equation (53) may be written as

$$
\Psi_{i}(t)=H^{\mathrm{T}}\left(t-t_{i}\right) b(t) \phi_{a_{i}}\left(t-t_{i}\right)
$$

To determine the shape functions, one has to select $n \geqslant 1$ and kernel function $\phi_{a_{i}}\left(t-t_{i}\right)$ with dilation parameters $\left\{a_{i}\right\}_{i=1}^{N p}$. The kernel function $\phi_{a_{i}}\left(t-t_{i}\right)$ is defined as

$$
\phi_{a_{i}}\left(t-t_{i}\right)=\frac{1}{a_{i}} \phi\left(\frac{t-t_{i}}{a_{i}}\right)=\frac{1}{a_{i}} \phi(s)
$$

where $s=\left(t-t_{i}\right) / a_{i}$. The kernel function $\phi(s)$ is presently taken as the cubic spline given by

$$
\phi(s)= \begin{cases}0, & s<-2 \\ \frac{1}{6}(s+2)^{3}, & -2 \leqslant s \leqslant-1 \\ \frac{2}{3}-s^{2}\left(1+\frac{s}{2}\right), & -1 \leqslant s \leqslant 0 \\ \frac{2}{3}-s^{2}\left(1-\frac{s}{2}\right), & 0 \leqslant s \leqslant 1 \\ \frac{1}{6}(s-2)^{3}, & 1 \leqslant s \leqslant 2 \\ 0, & s>2\end{cases}
$$


The vector of the coefficient functions $b\left(t_{i}\right)$ is determined by imposing the polynomial reproducing conditions. Consider the first $n+1$ terms of the Taylor series expansion for $u\left(t_{i}\right)$

$$
u\left(t_{i}\right)=u(t)-\left(t-t_{i}\right) \frac{\partial u}{\partial t}+\frac{\left(t-t_{i}\right)^{2}}{2 !} \frac{\partial^{2} u}{\partial t^{2}}-\cdots+(-1)^{n} \frac{\left(t-t_{i}\right)^{n}}{n !} \frac{\partial^{n} u}{\partial t^{n}}
$$

Substituting Equations (58), (61) in Equation (52), one obtains the following reproducing conditions to exactly reproduce any polynomial $u(t)$ of degree $n$ or less using $u^{a}\left(t_{i}\right)$ :

$$
\begin{aligned}
\sum_{i=1}^{N_{p}} H^{\mathrm{T}}\left(t-t_{i}\right) b(t) \phi_{a_{i}}\left(t-t_{i}\right) & =1 \\
\sum_{i=1}^{N_{p}} H^{\mathrm{T}}\left(t-t_{i}\right) b(t)\left(t-t_{i}\right)^{\alpha} \phi_{a_{i}}\left(t-t_{i}\right) & =0, \quad \alpha=1,2, \ldots, n
\end{aligned}
$$

Alternatively, these $n+1$ conditions may be expressed as

$$
\sum_{i=1}^{N_{p}} H\left(t-t_{i}\right) b(t) H^{\mathrm{T}}\left(t-t_{i}\right) \phi_{a_{i}}\left(t-t_{i}\right)=H(0)
$$

This leads to

$$
b(t)=M^{-1}(t) H(0)
$$

where

$$
M(t)=\sum_{i=1}^{N_{p}} H\left(t-t_{i}\right) H^{\mathrm{T}}\left(t-t_{i}\right) \phi_{a_{i}}\left(t-t_{i}\right)
$$

is called the moment matrix. Using Equations (58) and (64), the RK shape functions are finally obtained as

$$
\Psi_{i}(t)=H^{\mathrm{T}}(0) M^{-1}(t) H^{\mathrm{T}}\left(t-t_{i}\right) \phi_{a_{i}}\left(t-t_{i}\right)
$$

It is clear that the RKPM enables exact representations of polynomials of degree $n$ or lower in $\Omega$ and, for other functions, it affords an approximation of order $O\left(h^{n}\right)$.

We now extend the RKPM to address the problem of extrapolation (Figure 6). The problem on hand consists of estimating $\left\{u\left(t_{p+j}\right)\right\}_{j=1}^{q}$ given $\left\{u\left(t_{i}\right)\right\}_{i=1}^{p}$. Within the domain $\Omega \in\left[t_{1}, t_{p+q}\right], u(t)$ may be approximated using the RK method as

$$
u^{a}(t)=\sum_{i=1}^{p+q} \Psi_{i}(t) u_{i}=Q(t)+\sum_{i=1}^{q} \Psi_{p+i}(t) u_{p+i}
$$

where $u_{i}=u\left(t_{i}\right), u_{p+i}=u\left(t_{p+i}\right)$ and $Q(t)=\sum_{i=1}^{p} \Psi_{i}(t) u_{i}$. We note that the RK shape functions do not possess the interpolation property, that is $\Psi_{i}\left(t_{j}\right) \neq \delta_{i j}$. Therefore, $u^{a}\left(t_{i}\right) \neq u\left(t_{i}\right)$ and this 


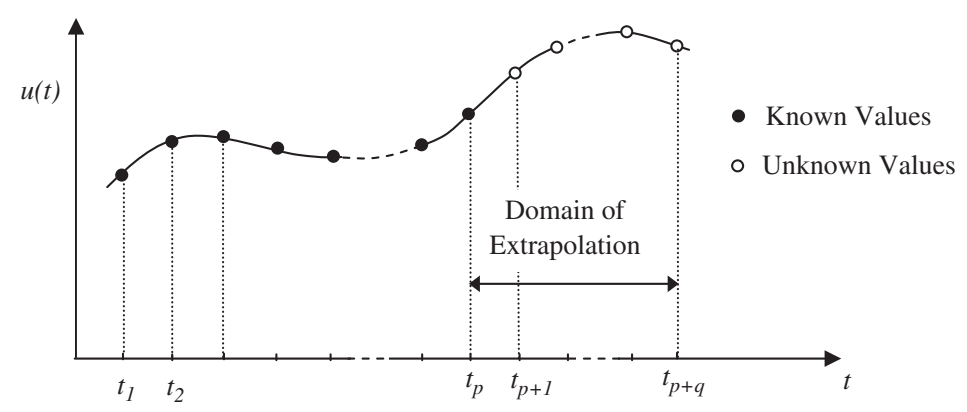

Figure 6. A schematic representation of multi-step extrapolations.

introduces a nodal error. In the extrapolation technique developed here, the unknown (extrapolated) values are determined by imposing conditions for minimizing this nodal error over $\Omega$.

The error at any node $i$ (corresponding to $t_{i}$ ) is

$$
e_{i}=u\left(t_{i}\right)-u^{a}\left(t_{i}\right)=u_{i}-Q_{i}-\sum_{j=1}^{q} \Psi_{p+j}\left(t_{i}\right) u_{i}
$$

where $Q_{i}=Q\left(t_{i}\right)$. A measure of total nodal error may be defined as

$$
E=\sum_{i=1}^{p} e_{i}^{2}
$$

Using Equation (68) in the above equation, we get

$$
\begin{aligned}
E= & \sum_{i=1}^{p}\left\{u_{i}^{2}+Q_{i}^{2}+\left(\sum_{j=1}^{q} \Psi_{p+j}\left(t_{i}\right) u_{p+j}\right)^{2}-2 u_{i} Q_{i}\right. \\
& \left.-2 u_{i} \sum_{j=1}^{q} \Psi_{p+j}\left(t_{i}\right) u_{p+j}+2 Q_{i} \sum_{j=1}^{q} \Psi_{p+j}\left(t_{i}\right) u_{p+j}\right\} \\
= & \sum_{i=1}^{p}\left(u_{i}^{2}+Q_{i}^{2}+\sum_{j=1}^{q} \sum_{k=1}^{q} \Psi_{p+j}\left(t_{i}\right) u_{p+j} \Psi_{p+k}\left(t_{i}\right) u_{p+k}-2 u_{i} Q_{i}\right. \\
& \left.-2 u_{i} \sum_{j=1}^{q} \Psi_{p+j}\left(t_{i}\right) u_{p+j}+2 Q_{i} \sum_{j=1}^{q} \Psi_{p+j}\left(t_{i}\right) u_{p+j}\right)
\end{aligned}
$$

The conditions for minimization of $E$ is obtained as

$$
\frac{\partial E}{\partial u_{p+r}}=0, \quad r \in[1, q]
$$


Following some algebraic manipulation, this leads to the condition

$$
\sum_{i=1}^{p}\left(2 \Psi_{p+r}^{2}\left(t_{i}\right) u_{p+r}+2 \sum_{\substack{s=1 \\ s \neq r}}^{q} \Psi_{p+r}\left(t_{i}\right) \Psi_{p+s}\left(t_{i}\right) u_{p+s}-2 u_{i} \Psi_{p+r}\left(t_{i}\right)+2 Q_{i} \Psi_{p+r}\left(t_{i}\right)\right)=0
$$

These equations can be recast in a matrix form as

$$
\tilde{A} \tilde{U}=\tilde{B}
$$

where

$$
\begin{aligned}
& \tilde{A}=\left[\begin{array}{ccccc}
\sum_{i=1}^{p} \Psi_{p+1}^{2}\left(t_{i}\right) & \sum_{i=1}^{p} \Psi_{p+1}\left(t_{i}\right) \Psi_{p+2}\left(t_{i}\right) & \ldots & \ldots & \sum_{i=1}^{p} \Psi_{p+1}\left(t_{i}\right) \Psi_{p+q}\left(t_{i}\right) \\
& \sum_{i=1}^{p} \Psi_{p+2}^{2}\left(t_{i}\right) & \cdots & \cdots & \sum_{i=1}^{p} \Psi_{p+2}\left(t_{i}\right) \Psi_{p+q}\left(t_{i}\right) \\
& & & & \vdots \\
\text { Symmetric } & & \ddots & \vdots \\
& & & & \sum_{i=1}^{p} \Psi_{p+q}^{2}\left(t_{i}\right)
\end{array}\right], \tilde{A} \in \mathfrak{i}^{q \times q}
\end{aligned}
$$

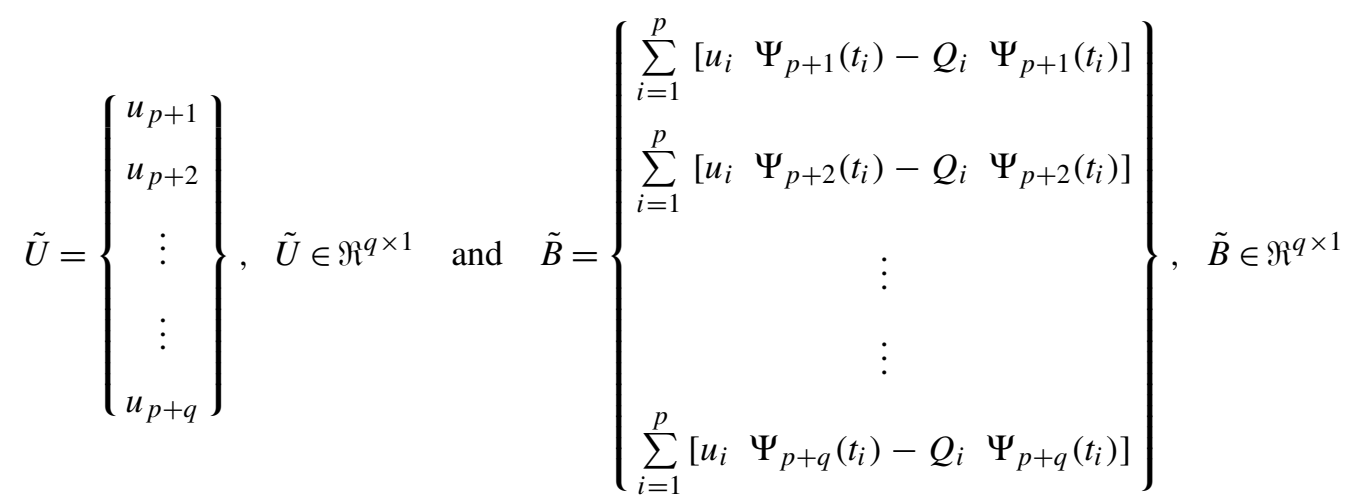

Once the extrapolated nodal values $\left\{u\left(t_{p+j}\right)\right\}_{j=1}^{q}$ are determined from the above equations, $u^{a}(t)$ at any time $t$ may be subsequently calculated using Equation (67).

To illustrate the relative performance of the RK-based method and the Lagrangian polynomialbased method for extrapolation, we consider the function $u(t)=10 \sin (70 t)+5 \cos (10 t)$ defined over $\Omega \in[0,0.12]$. It is assumed that values of $u(t)$ for $t \leqslant 0.09$ is known for $t=0,0.01, \ldots, 0.09$. The solution to this problem is obtained using the RK-based method with $p=10$ and $q=3$ and Lagrangian polynomial-based extrapolation with orders $r=4$ and 9. It is of interest to note that an $r$ th order Lagrangian polynomial method uses $r+1$ past values for extrapolation. The superior 

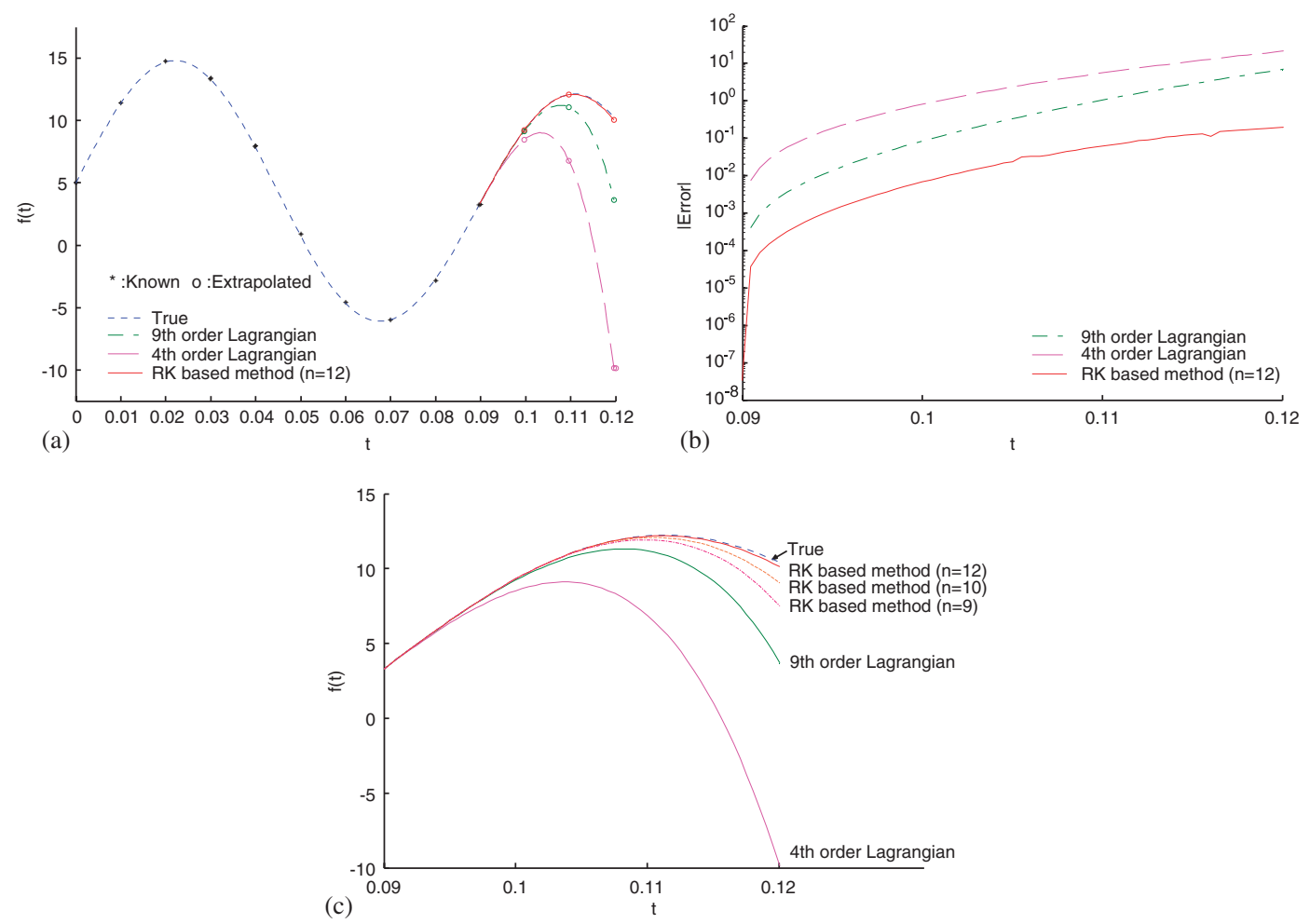

Figure 7. Extrapolation using RK-based method and Lagrangian polynomials: (a) the function $u(t)=10 \sin (70 t)+5 \cos (10 t)$; (b) error in extrapolation (calculated as $\left.u(t)-u^{a}(t)\right)$; and (c) enlarged view of the extrapolated function.

performance of the RK-based method is evident from Figures 7(a)-(c) in which the results of extrapolation using the two schemes are reported.

\subsection{Implementation of the RTS algorithm}

The implementation of the RTS algorithm involves the following major steps.

I. The iterative phase (non-real time)

1. Choose an appropriate $T_{\mathrm{I}}>t_{0}$ such that iterative computations are completed during the interval $\left(t_{0}, T_{\mathrm{I}}\right]$. Choose a suitable $p$ and divide $\left(t_{0}, T_{\mathrm{I}}\right]$ into $p-1$ subdivisions. Choose an appropriate value of $s$ and divide each time step to $s$ subdivisions.

2. Consider $S^{\mathrm{I}}$ over $i$ th time step $\left(t_{i-1}, t_{i}\right]$ and $j$ th iteration. Find $G^{\mathrm{I}, j-1}(t)$ and $Q^{\mathrm{I}, j-1}(t)$ and use them along with $F^{\mathrm{I}}(t)$ to find the response $Y^{\mathrm{I}, j}(t)$ at the finer grid points (defined by $s$ ).

3. Based on the values of $Y^{\mathrm{I}}$ obtained in step (2), apply the displacements corresponding to the interface dofs on $S^{\mathrm{II}}$ using suitable actuation devices, while subjecting it also to any dynamic loads that may be present. Measure the components of $G^{\mathrm{I}}(t)$ at the interface through transducers and a data acquisition system. 
4. Go back to steps (2) and (3) for increasing $j$ till $\left\|Y^{\mathrm{I}, j+1}-Y^{\mathrm{I}, j}\right\|<\varepsilon$ at all fine grid points. 5. Repeat steps 2,3 and 4 for all $i \in[1, p]$.

\section{The non-iterative phase (real time)}

6. Choose an appropriate value of $q$. Consider the time duration $\left(t_{i}, t_{i+q}\right]$ for $i \geqslant p$. Start with $S^{\mathrm{I}}$. Using the values of $G^{\mathrm{I}}(t)$ and $Q^{\mathrm{I}}(t)$ at $\left\{t_{i-(p-1)}, \ldots, t_{i}\right\}$, extrapolate $G^{\mathrm{I}}(t)$ and $Q^{\mathrm{I}}(t)$ over $\left(t_{i}, t_{i+q}\right]$. Obtain the response $Y^{\mathrm{I}}(t)$ over $\left(t_{i}, t_{i+q}\right]$.

7. From the values of $Y^{\mathrm{I}}(t)$ obtained in step (6), apply the displacements, corresponding to the interface dofs, on $S^{\mathrm{II}}$. Measure the components of the interactive force $G^{\mathrm{I}}(t)$ at the interface and store these values.

8. Repeat steps 6 and 7 with $i=i+q$ till the RTS-based simulation is complete.

\section{NUMERICAL EXAMPLES}

For purposes of validation of the RTS method, only a numerical route is presently chosen. A few low-dimensional linear and non-linear oscillators under support motion are considered and the RTS method is applied by treating them as a collection of numerical substructures only. For the illustrations of RTS method for systems with linear substructures, we have used the explicit scheme outlined in Section 2. To start with, we consider a two degree-of-freedom (TDOF) linear oscillator under a sinusoidal support motion. The equations of motion are:

$$
\begin{aligned}
m_{1} \ddot{x}+c_{1}(\dot{x}-\dot{u})+c_{2}(\dot{x}-\dot{y})+k_{1}(x-u)+k_{2}(x-y) & =0 \\
m_{2} \ddot{y}+c_{2}(\dot{y}-\dot{x})+k_{2}(y-x) & =0
\end{aligned}
$$

The support motion is given by $u(t)=A \cos (\lambda t)$ and the initial conditions are $x(0)=x_{0}, \dot{x}(0)=\dot{x}_{0}$, $y(0)=y_{0}$ and $\dot{y}(0)=\dot{y}_{0}$. The system is represented in Figure 8(a) as a collection of two springmass-damper units and the free-body diagrams of these units are also indicated. The system is split into two numerical substructures, being subjected to two separate support motions (Figure 8(b)). The sub-structural equations of motion are

$$
\begin{aligned}
& m_{1} \ddot{x}+c_{1} \dot{x}+k_{1} x=f_{1}(t)+G^{\mathrm{I}}(y, \dot{y}, t) \\
& m_{2} \ddot{y}+c_{2} \dot{y}+k_{2} y=G^{\mathrm{II}}(x, \dot{x}, t)
\end{aligned}
$$

where $f_{1}(t)=k_{1} u+c_{1} \dot{u}$ is the externally applied force acting on the substructure I due to support motion, $G^{\mathrm{I}}=k_{2}(y-x)+c_{2}(\dot{y}-\dot{x})$ and $G^{\mathrm{II}}=k_{2} x+c_{2} \dot{x}$ are the interactive forces acting on substructures I and II, respectively. The RTS simulation starts with an iterative method applied successively to NS-I and NS-II. Presently only linear problems are being tackled and the kind of loading considered does not lead to strong local characteristics in the response. Thus, a uniform step size, $h_{i}=t_{i}-t_{i-1}=h$ for all $i=1,2, \ldots$, is chosen for all numerical results. If we consider the first time interval $\left(t_{0}, t_{1}\right]$, the 0th iterative estimate of $G^{\mathrm{I}}$ acting on NS-I is given by (see Equation (7a))

$$
G^{\mathrm{I}, 0}=k_{2}\left(x_{0}-y_{0}\right)+c_{2}\left(\dot{x}_{0}-\dot{y}_{0}\right)
$$




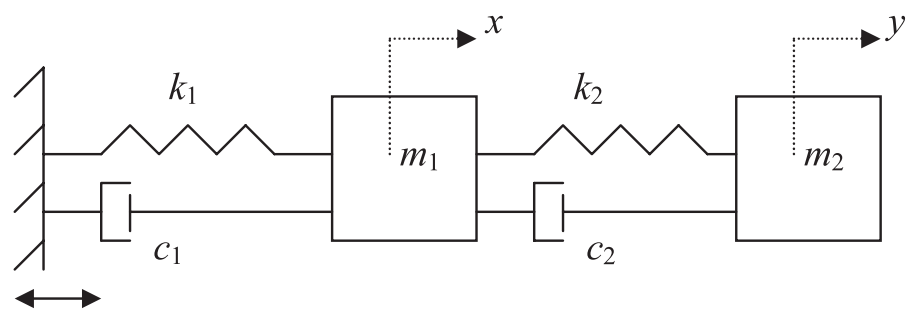

$u(t)$

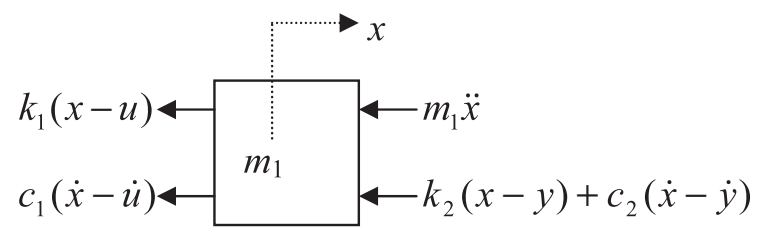

$k_{2}(y-x) \longleftarrow m_{2}$

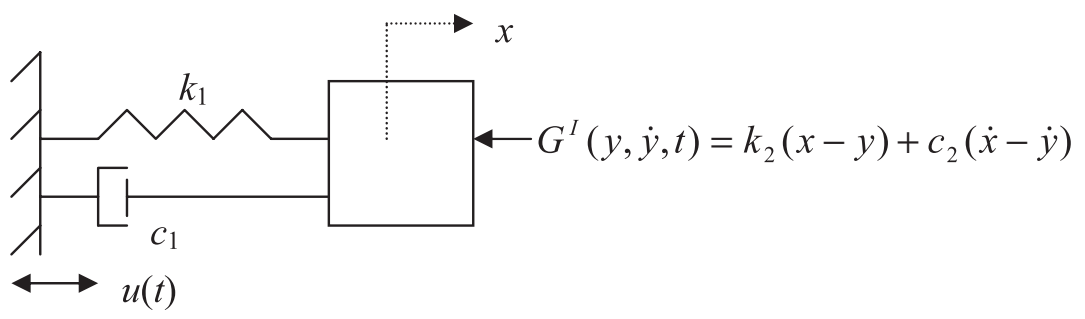

Numerical Substructure I (NS-I)

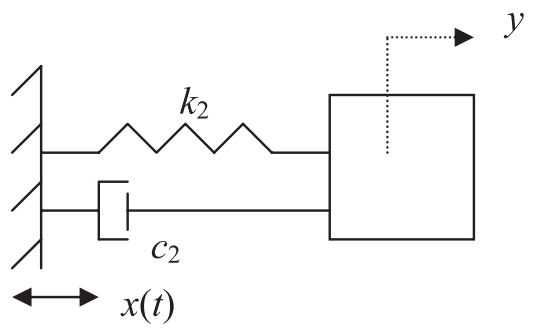

(b)

\section{Numerical Substructure II (NS-II)}

Figure 8. (a) A two-degree-of-freedom linear oscillator under support motion and the associated free-body diagrams and (b) two numerical substructures representing the two-degree-of-freedom linear oscillator under support motion within the RTS framework. 
Note that in case NS-II is treated as a test substructure, the interactive force $G^{\mathrm{I}, 0}$ cannot be determined through the equation above as no information on $k_{2}$ and $c_{2}$ is available. In such a case, the interactive force is determined by applying an initial displacement $\left(y_{0}-x_{0}\right)$ and initial velocity $\left(\dot{y}_{0}-\dot{x}_{0}\right)$ (or an equivalent effective force) to the substructure II followed by the measurement of the force generated at its support. Presently, the equation governing NS-I is solved under the combined forcing function $f_{1}(t)+G^{\mathrm{I}, 0}$ using Equation (9a), where the system coefficient matrix is given by

$$
A^{\mathrm{I}}=\left[\begin{array}{cc}
0 & 1 \\
-k_{1} / m_{1} & -c_{1} / m_{1}
\end{array}\right]
$$

This leads to the first iterative approximation $\left(x^{\mathrm{I}, 1}, \dot{x}^{\mathrm{I}, 1}\right)^{\mathrm{T}}$ to the response $(x, \dot{x})^{\mathrm{T}}$ for NS-I for $t \in\left(t_{0}, t_{1}\right]$. The first iterate $G^{\mathrm{II}, 1}$ to $G^{\mathrm{II}}$ is now constructed as

$$
G^{\mathrm{II}, 1}=k_{2} x^{\mathrm{I}, 1}+c_{2} \dot{x}^{\mathrm{I}, 1}
$$

The first iterate $\left(y^{\mathrm{II}, 1}, \dot{y}^{\mathrm{II}, 1}\right)^{\mathrm{T}}$ for the response vector $\left(y, \dot{y} \mid t \in\left(t_{0}, t_{1}\right]\right)^{\mathrm{T}}$ is then computed using Equation (12a) with the system coefficient matrix of NS-II given by

$$
A^{\mathrm{II}}=\left[\begin{array}{cc}
0 & 1 \\
-k_{2} / m_{2} & -c_{2} / m_{2}
\end{array}\right]
$$

The solution vector $\left(y^{\mathrm{II}, 1}, \dot{y}^{\mathrm{II}, 1}\right)^{\mathrm{T}}$ for NS-II allows constructing the first iterate $G^{\mathrm{I}, 1}(t)=k_{2}\left(x^{\mathrm{I}, 1}(t)-\right.$ $\left.y^{\mathrm{II}, 1}(t)\right)+c_{2}\left(\dot{x}^{\mathrm{I}, 1}(t)-\dot{y}^{\mathrm{II}, 1}(t)\right)$ and thus obtain $\left(x^{\mathrm{I}, 2}, \dot{x}^{\mathrm{I}, 2}\right)^{\mathrm{T}}$, which is the second iterative approximation to $\left(x^{\mathrm{I}}, \dot{x}^{\mathrm{I}}\right)^{\mathrm{T}}$. Using $p_{l}=4$, it suffices to have just three iterations to achieve the required order of accuracy. Moreover, it is necessary to carry out iterative response computations over the first $p_{l}-1$ time steps before starting the non-iterative procedure. Thus for $p_{l}=4$ with constant $h$, iterative computations must be performed over the first three steps, i.e. over $t \in\left(t_{0}, t_{3}\right]$ and the non-iterative process takes over for $t \in\left(t_{3}, T\right]$, where $T$ denotes the final instant at which the RTS-based simulation must be stopped. In order to apply the non-iterative procedure, it is necessary to obtain approximations $G^{\mathrm{I}, p_{l}}$ and $G^{\mathrm{II}, p_{l}}$ through the interpolation schemes given by Equations (27), (28) and (32). For instance, the first interval of application of the non-iterative method with $p_{l}=4$ is $\left(t_{3}, t_{4}\right]$ and the substructural equation of motion (75a) for NS-I is solved over this interval with $G^{\mathrm{I}}$ approximated by $G^{\mathrm{I}, 4}$

$$
G^{\mathrm{I}, 4}(t)=\sum_{j=0}^{3} k_{2}\left(x\left(t_{j}\right)-y\left(t_{j}\right)\right)+c_{2}\left(\dot{x}\left(t_{j}\right)-\dot{y}\left(t_{j}\right)\right) \prod_{\substack{l=0 \\ l \neq j}}^{3} \frac{\left(t-t_{l}\right)}{\left(t_{j}-t_{l}\right)}, \quad t \in\left(t_{3}, t_{4}\right]
$$

A similar approximation to $G^{\mathrm{II}}$ and a subsequent solution of the sub-structural equation of motion for NS-II are also performed for completing the non-iterative procedure over $\left(t_{3}, t_{4}\right]$. We consistently assume $h=0.01$ and $p_{l}=4$ unless otherwise mentioned explicitly. Figures 9-13 show some response histories of the oscillator via RTS and an exact state-space formulation for different mass and frequency ratios $\mu=m_{2} / m_{1}, \alpha_{0}=\sqrt{k_{2} / m_{2}} / \sqrt{k_{1} / m_{1}}$. It is known [54] that for $\mu \ll 1$ and 

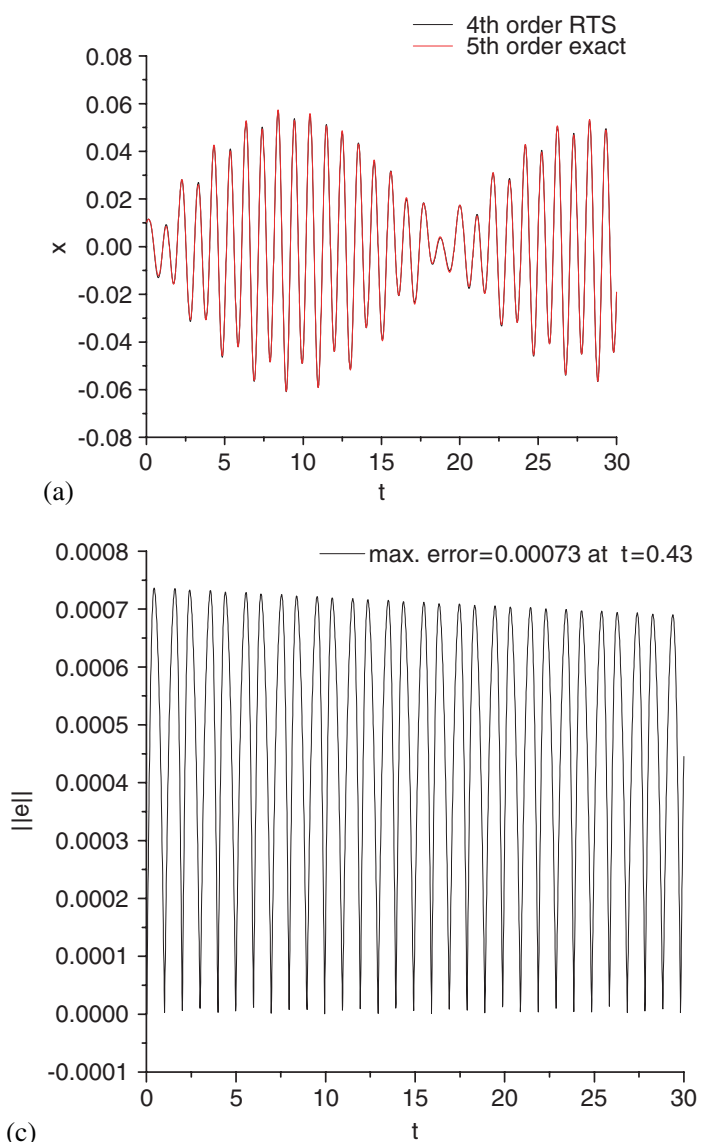
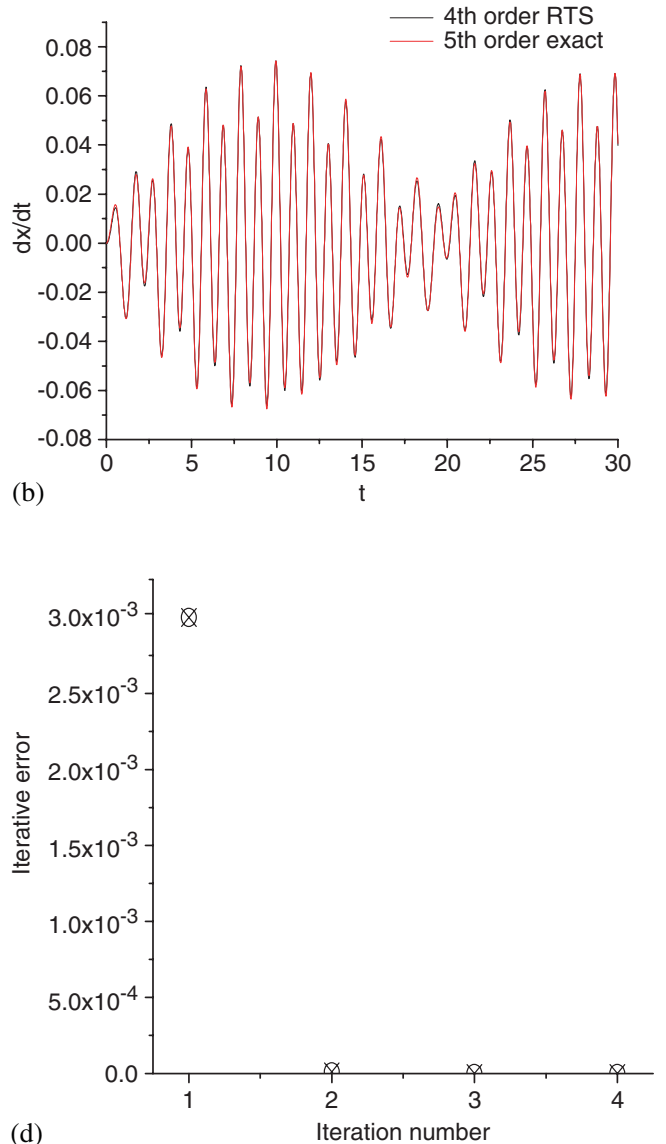

Figure 9. 2-DOF oscillator under support motion; $m_{1}=10.0, m_{2}=5.0$ (i.e. $\mu=0.5$ ), $k_{1}=200.0, k_{2}=100.0$ (i.e. $\left.\alpha_{0}=1.0\right), c_{1}=0.05, c_{2}=0.06, A=0.01, \lambda=6.0$ : (a) displacement history; (b) velocity history; (c) Euclidean error norm history; and (d) iteration error (during the first step of application of the iterative method) with iteration numbers.

$\alpha_{0}$ remaining far away from 1 , dynamic interactions between the two substructures are small. The less the interactions, the more similar is the likelihood of the two substructures behaving as a pair of uncoupled single-degree-of-freedom (SDOF) oscillators. Within the RTS framework, this implies that effects of the interactive forcing functions $G^{\mathrm{I}}$ and $G^{\mathrm{II}}$ remain small, or even insignificant, on the responses of substructures I and II, respectively. Figure 9(a) shows a case of strong dynamic interactions with $\mu=0.5$ and $\alpha_{0}=1$. That the interactive forces play a significant role in determining the displacement and velocity histories becomes quite apparent from the beating oscillations. In case the dynamical interactions are minimal, we expect NS-I to respond sinusoidally (as the support excitation is sinusoidal) and NS-II would barely get excited. On the other hand, as shown in Figures 9(a) and (b), if strong dynamic interactions are present, the sub-structural response may be fundamentally different from that without the interactive forces. 

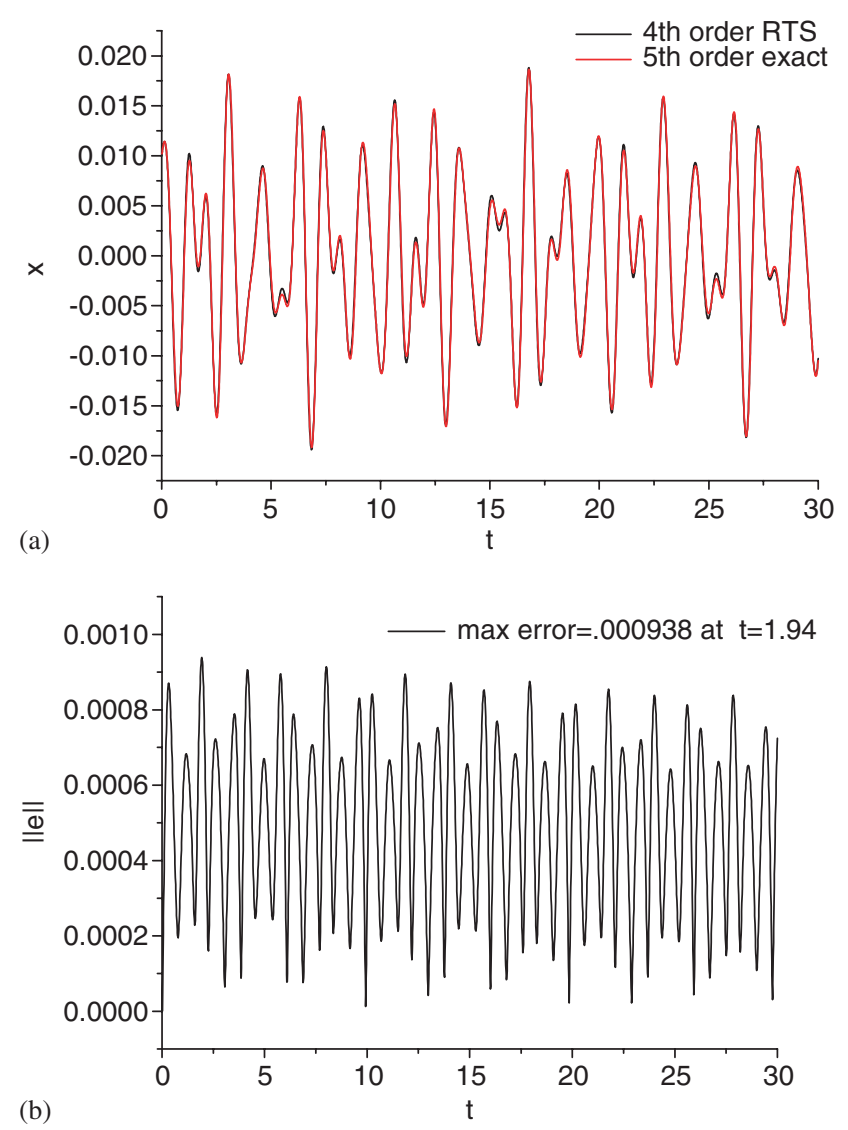

Figure 10. 2-DOF oscillator under support motion; $m_{1}=10.0, m_{2}=\frac{10}{3}$ (i.e. $\mu=0.3$ ), $k_{1}=300.0, k_{2}=100.0$ (i.e. $\alpha_{0}=1.0$ ), $c_{1}=0.05, c_{2}=0.06, A=0.01, \lambda=6.0$ : (a) displacement history and (b) Euclidean error norm history.

Comparisons with the 'true' solution, with terms up to order $O\left(h^{5}\right)$ included (herein referred to as '5th order exact solution'), are also provided. Time history of the instantaneous error norm, presently expressed as

$$
\|e(t)\|=\left\{\left(x(t)-x^{\mathrm{R}}(t)\right)^{2}+\left(y(t)-y^{\mathrm{R}}(t)\right)^{2}+\left(\dot{x}(t)-\dot{x}^{\mathrm{R}}(t)\right)^{2}+\left(\dot{y}(t)-\dot{y}^{\mathrm{R}}(t)\right)^{2}\right\}^{1 / 2}
$$

is shown in Figure 9(c). For this case, the history is almost like a decreasing sinusoid with very low noisy components. It has been numerically observed that noise (i.e. unpredictable/uncertain components) in $\|e\|$ is less whenever there are strong dynamic interactions among the substructures. In Figure 9(d), the iterative error norm over the first time step, $\left(t_{0}, t_{1}\right]$, expressed as

$$
I_{\mathrm{E}}^{j}=0.5\left(\left\langle\left\|\hat{J}^{\mathrm{I}, j}(t)\right\|\right\rangle+\left\langle\left\|\hat{J}^{\mathrm{II}, j}(t)\right\|\right\rangle\right)
$$




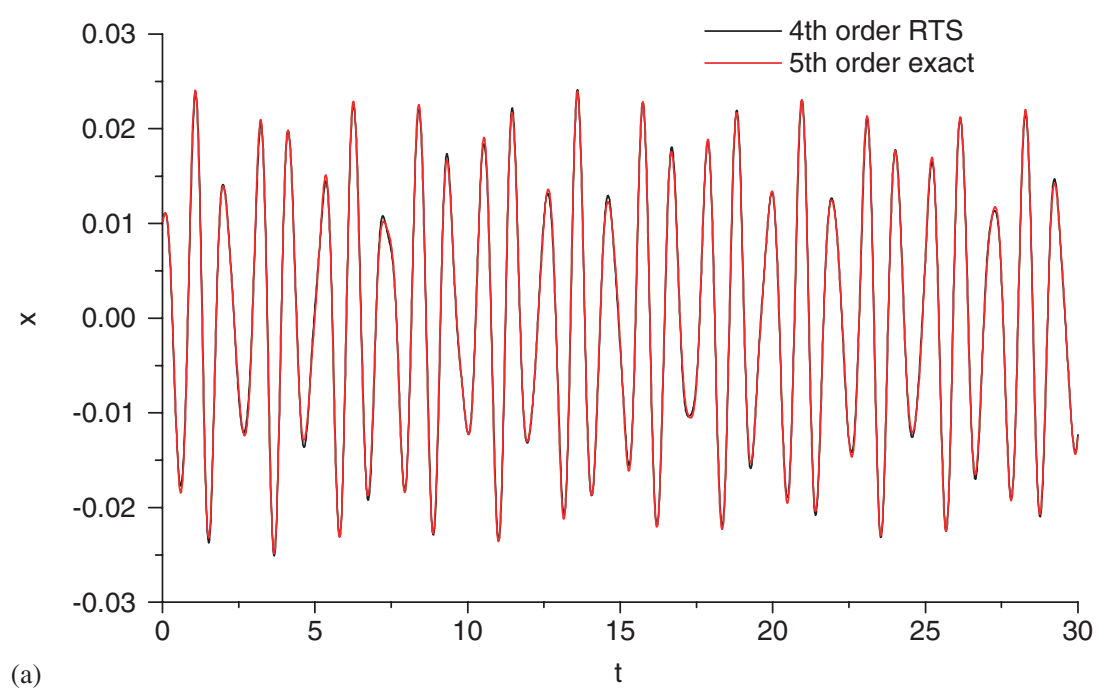

(a)

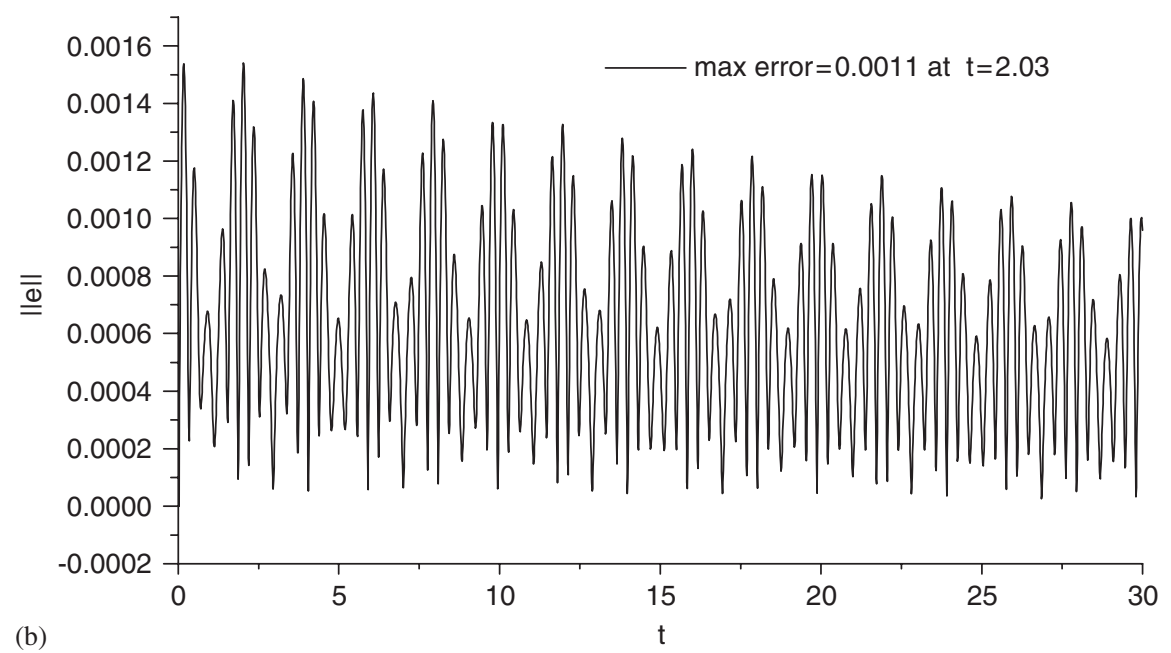

Figure 11. 2-DOF oscillator under support motion; $m_{1}=10.0, m_{2}=1.0$ (i.e. $\mu=0.1$ ), $k_{1}=1000.0$, $k_{2}=100.0$ (i.e. $\alpha_{0}=1.0$ ), $c_{1}=0.05, c_{2}=0.06, A=0.01, \lambda=6.0$ : (a) displacement history and (b) Euclidean error norm history.

is plotted as a function of the iteration number, $j$. It is clear that the iteration error drops very rapidly with increasing $j$. It is noted that while there is an actual control of error during the iteration stage, the control of error in non-iterative scheme is merely formal and one simply hopes to keep it low through an appropriate choice of $p_{l}$. Thus, it may be worth exploring the possibility of implementing the RTS method only via the iterative strategy. However, this issue is beyond the scope of this work and will be taken up elsewhere. Figures 10-13 consider a few other cases of the 2-DOF system with gradually decreasing dynamic interactions among NS-I and NS-II. It is interesting to observe that with decreasing dynamic interactions the history of the 


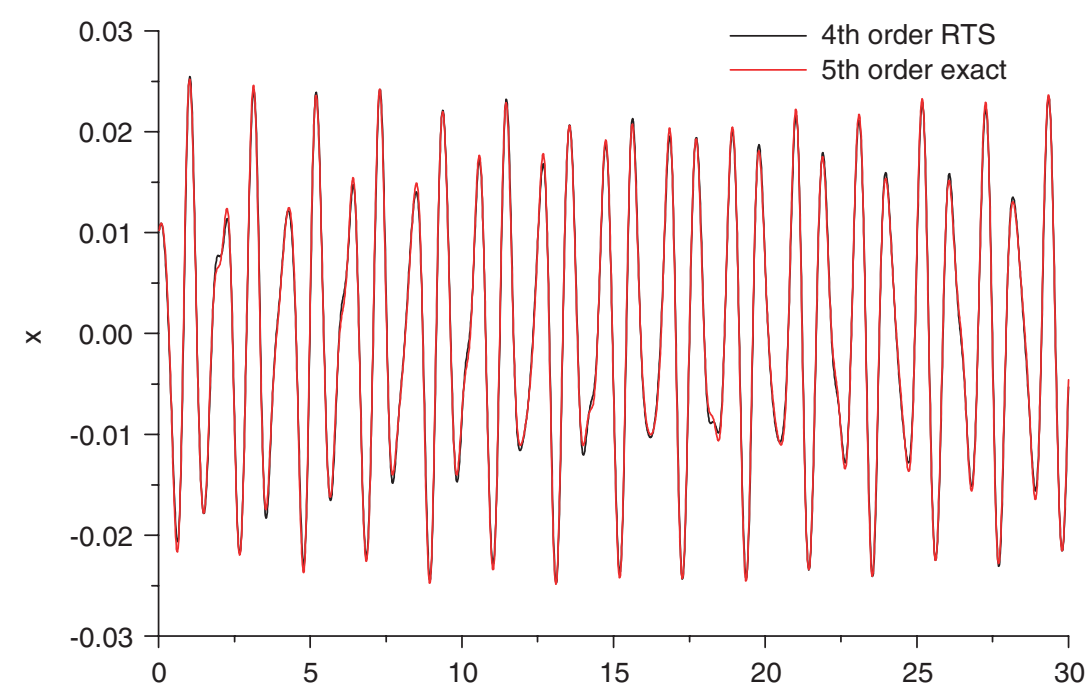

(a)

$\mathrm{t}$

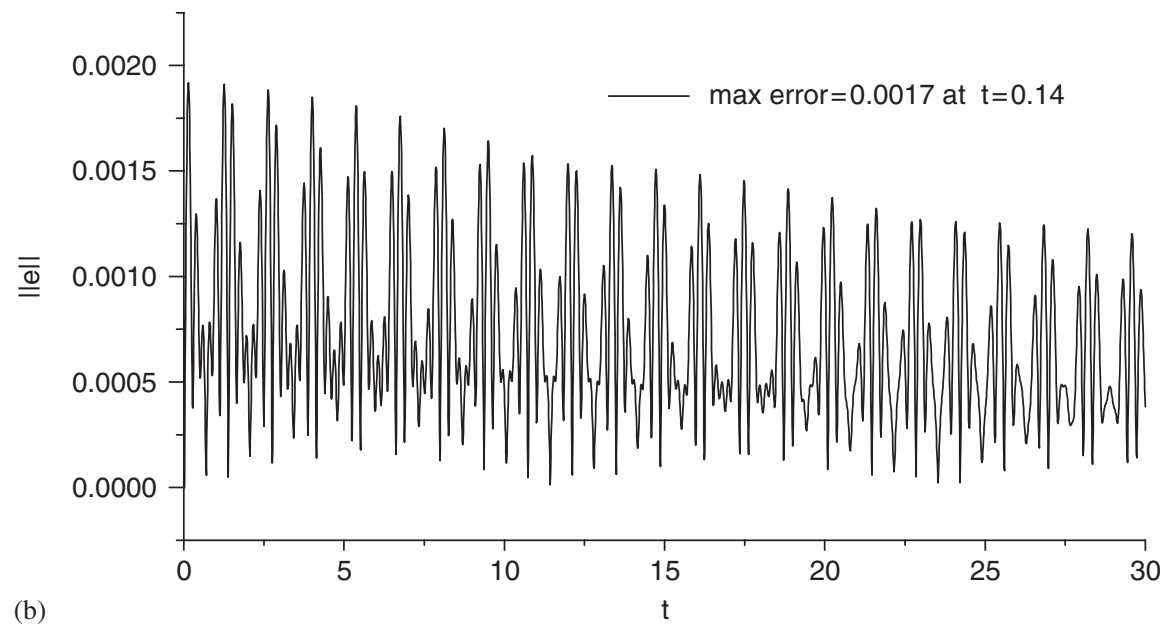

Figure 12. 2-DOF oscillator under support motion; $m_{1}=10.0, m_{2}=1.0$ (i.e. $\mu=0.1$ ), $k_{1}=1000.0$, $k_{2}=156.25$ (i.e. $\alpha_{0}=1.25$ ), $c_{1}=0.05, c_{2}=0.06, A=0.01, \lambda=6.0$ : (a) displacement history and (b) Euclidean error norm history.

instantaneous error norm appears to become increasingly noisy during the transient regime. One possible explanation for this could be that decreasing dynamic interactions could imply a possibility of interactions at higher frequency levels with lower amplitudes. However, $\|e(t)\|$ nearly goes to zero when the substructures exhibit steady-state solutions. This is shown in Figure 13, wherein the dynamic interactions are very less and after some time the substructures exhibit nearly sinusoidal motion in the steady state. 

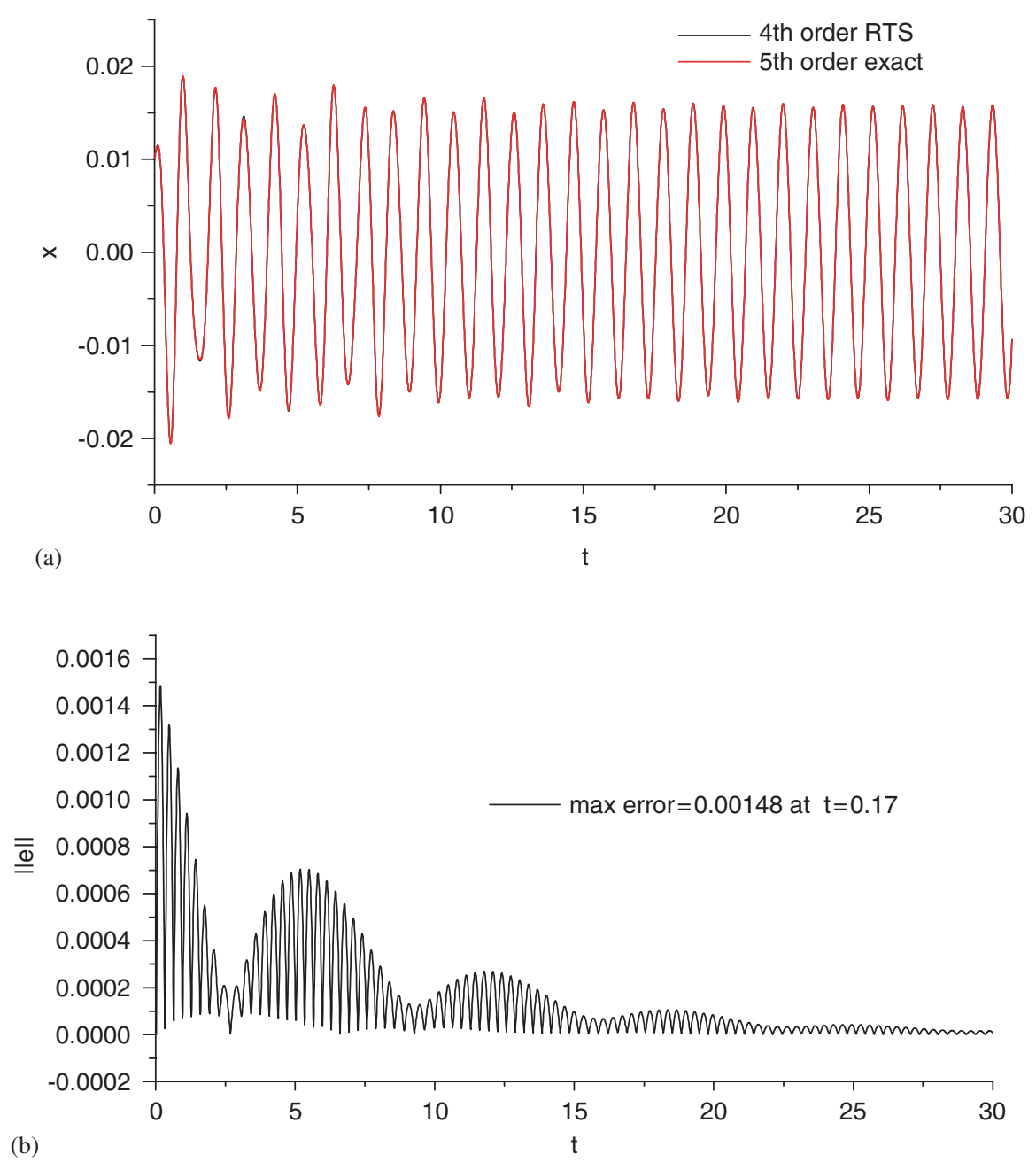

Figure 13. 2-DOF oscillator under support motion; $m_{1}=10.0, m_{2}=0.1$ (i.e. $\mu=0.01$ ), $k_{1}=1000.0$, $k_{2}=10.0$ (i.e. $\alpha_{0}=1.0$ ), $c_{1}=0.05, c_{2}=0.06, A=0.01, \lambda=6.0$ : (a) displacement history and (b) Euclidean error norm history.

Next, the response of the 2-DOF oscillator under a sample stationary stochastic support acceleration is considered; see Figures $14\left(\alpha_{0}=1, \mu=0.5\right)$ and $9\left(\alpha_{0}=1, \mu=0.01\right)$. The excitation power spectral density (PSD) function, defined for positive values of frequency, is taken to have a constant value of $G_{0}=2 \pi \sigma^{2} / \omega_{\max }$ with $\sigma=0.1 \mathrm{~g}, \omega_{\max }=60 \pi \mathrm{rad} / \mathrm{s}, g=$ acceleration due to gravity, $9.8 \mathrm{~m} / \mathrm{s}^{2}$, with the lowest frequency being zero. Figure 14(a) shows a sample time history that is compatible with this PSD function with $\Delta t=\frac{1}{80} \mathrm{~s}$. Satisfactory performance of RTS simulations is evidenced from Figures 14(b) and (c), and 15(a)-(c).

A three-storied building frame carrying a singly supported subsystem and a doubly supported subsystem is considered next; see Figure 16(a). Captions to Figures 17 and 18 contain the details of the system properties considered. A lumped parameter model for the complete system (Figure 16(b)) 

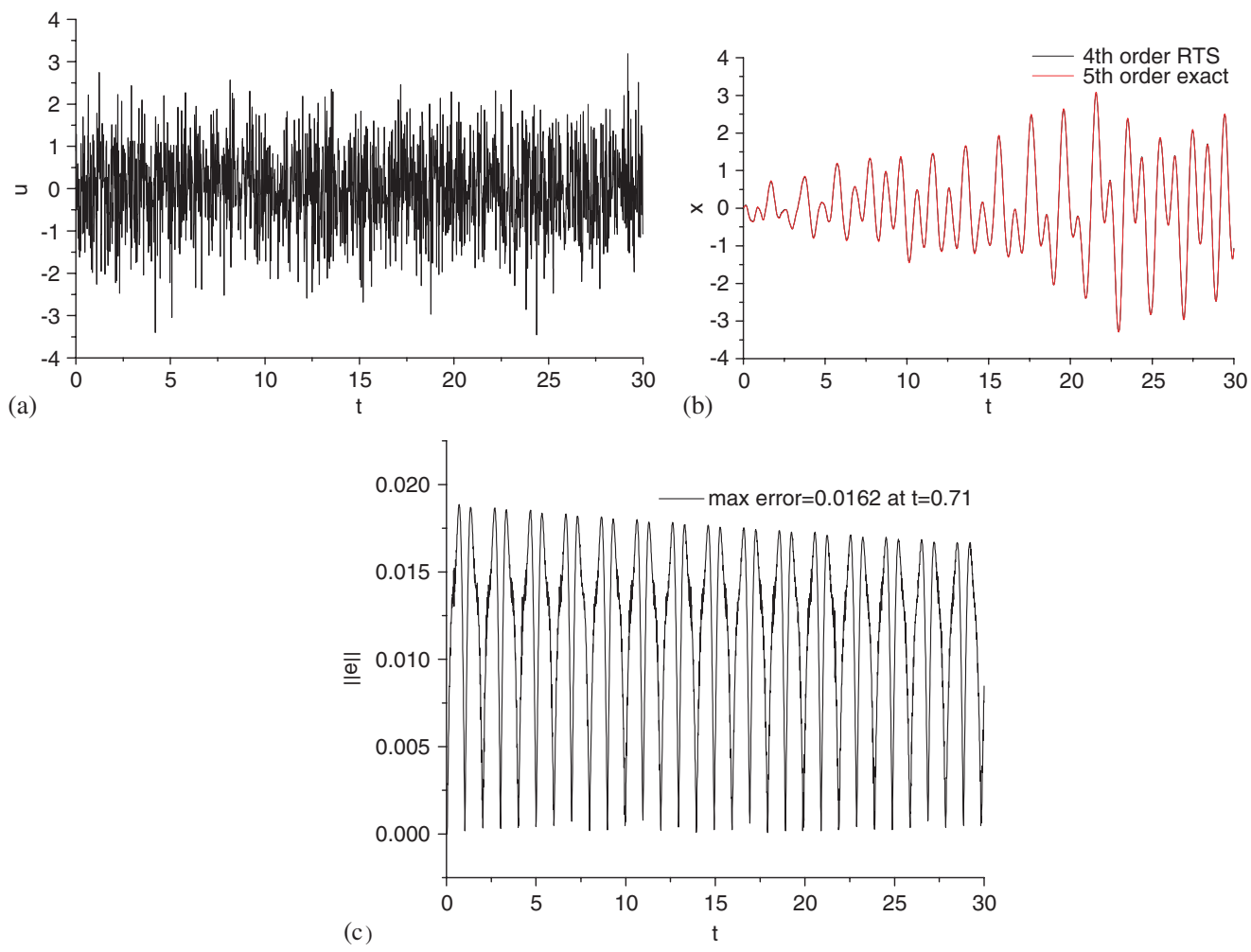

Figure 14. 2-DOF oscillator under a stationary, random support motion; $m_{1}=10.0$, $m_{2}=5.0$ (i.e. $\mu=0.5$ ), $k_{1}=200.0, k_{2}=100.0$ (i.e. $\alpha_{0}=1.0$ ), $c_{1}=0.05, c_{2}=0.06, N=3000$, $\omega_{\max }=60 \pi ; \Delta \omega=0.02 \pi, \sigma=0.1 \mathrm{~g}\left(\mathrm{~g}=9.81 \mathrm{~m} / \mathrm{s}^{2}\right)$ : (a) history of support motion; (b) displacement history; and (c) Euclidean error norm history.

yields the following equation of motion:

$$
\begin{aligned}
& {\left[\begin{array}{ccccc}
m_{1} & 0 & 0 & 0 & 0 \\
0 & m_{2} & 0 & 0 & 0 \\
0 & 0 & m_{3} & 0 & 0 \\
0 & 0 & 0 & m_{4} & 0 \\
0 & 0 & 0 & 0 & m_{5}
\end{array}\right]\left\{\begin{array}{l}
\ddot{x}_{1} \\
\ddot{x}_{2} \\
\ddot{x}_{3} \\
\ddot{x}_{4} \\
\ddot{x}_{5}
\end{array}\right\}+\left[\begin{array}{ccccc}
c_{1}+c_{2}+c_{5} & -c_{2} & 0 & 0 & 0 \\
-c_{2} & c_{2}+c_{3} & -c_{3} & 0 & 0 \\
0 & -c_{3} & c_{3}+c_{4}+c_{6} & -c_{6} & -c_{4} \\
0 & 0 & -c_{6} & c_{5}+c_{6} & 0 \\
0 & 0 & -c_{4} & 0 & c_{4}
\end{array}\right] \times\left\{\begin{array}{c}
\dot{x}_{1} \\
\dot{x}_{2} \\
\dot{x}_{3} \\
\dot{x}_{4} \\
\dot{x}_{5}
\end{array}\right\}} \\
& +\left[\begin{array}{ccccc}
k_{1}+k_{2}+k_{5} & -k_{2} & 0 & 0 & 0 \\
-k_{2} & k_{2}+k_{3} & -k_{3} & 0 & 0 \\
0 & -k_{3} & k_{3}+k_{4}+k_{6} & -k_{6} & -k_{4} \\
0 & 0 & -k_{6} & k_{5}+k_{6} & 0 \\
0 & 0 & -k_{4} & 0 & k_{4}
\end{array}\right]\left\{\begin{array}{c}
x_{1} \\
x_{2} \\
x_{3} \\
x \\
x_{5}
\end{array}\right\}=\left\{\begin{array}{c}
k_{1} u+c_{1} \dot{u} \\
0 \\
0 \\
0 \\
0
\end{array}\right\}
\end{aligned}
$$

To implement the RTS simulations, we treat the building frame as the numerical model and the two subsystems as two separate test substructures; see Figures 16(c)-(f). The governing equations 

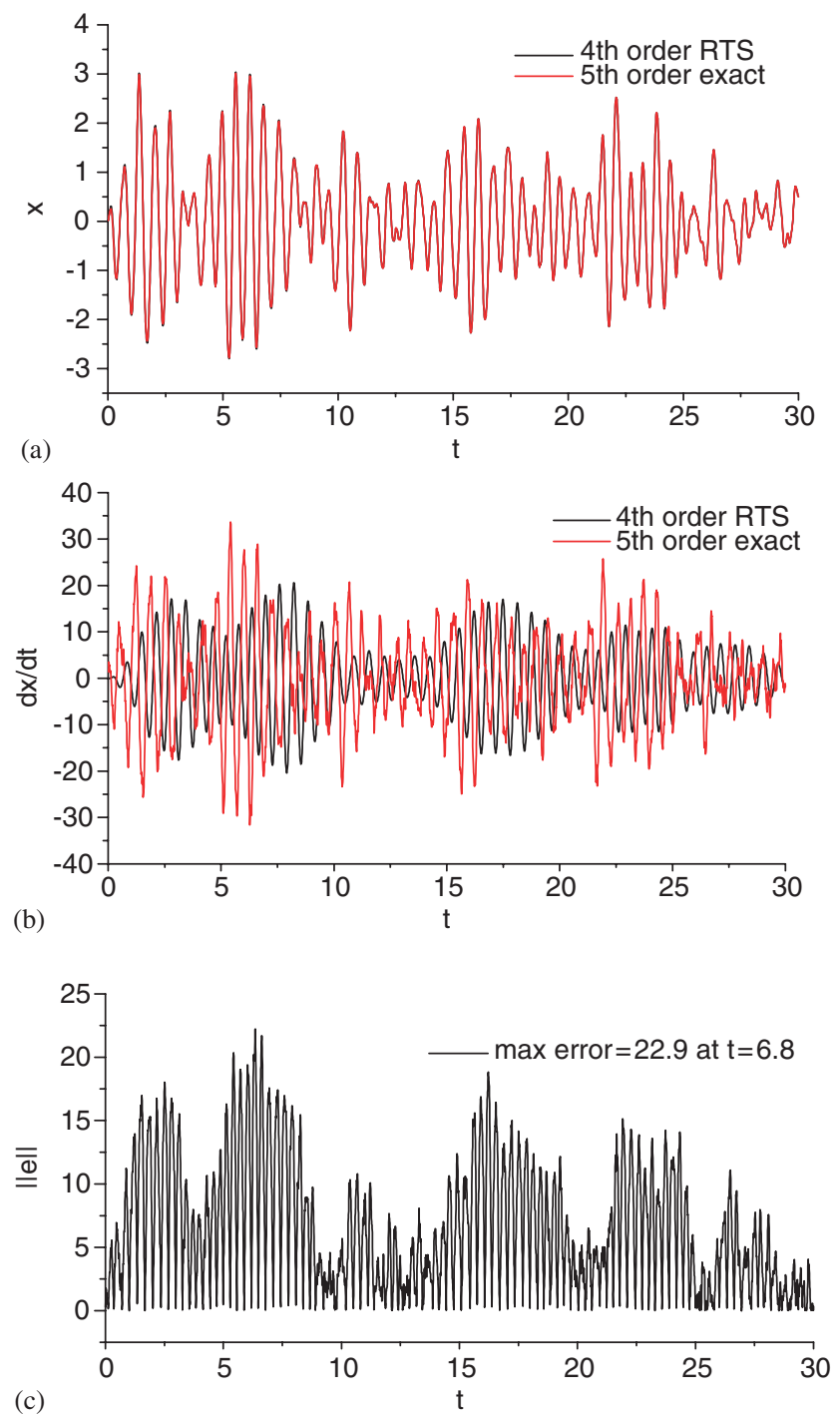

Figure 15. 2-DOF oscillator under a stationary, random support motion; $m_{1}=10.0, m_{2}=0.1$ (i.e. $\mu=0.01$ ), $k_{1}=1000.0, k_{2}=10.0$ (i.e. $\alpha=1.0$ ), $c_{1}=0.05, c_{2}=0.06, N=3000, \omega_{\max }=60 \pi ; \Delta \omega=0.02 \pi, \sigma=0.1 \mathrm{~g}$ $\left(g=9.81 \mathrm{~m} / \mathrm{s}^{2}\right)$ : (a) displacement history; (b) velocity history; and (c) Euclidean error norm history.

of motion for the numerical substructure in this case are obtained as

$$
\begin{gathered}
\left.\left[\begin{array}{ccc}
m_{1} & 0 & 0 \\
0 & m_{2} & 0 \\
0 & 0 & m_{3}
\end{array}\right]\left\{\begin{array}{l}
\ddot{x}_{1} \\
\ddot{x}_{2} \\
\ddot{x}_{3}
\end{array}\right\}+\left[\begin{array}{ccc}
c_{1}+c_{2} & -c_{2} & 0 \\
-c_{2} & c_{2}+c_{3} & -c_{3} \\
0 & -c_{3} & c_{3}
\end{array}\right]\right\}\left\{\begin{array}{c}
\dot{x}_{1} \\
\dot{x}_{2} \\
\dot{x}_{3}
\end{array}\right\} \\
+\left[\begin{array}{ccc}
k_{1}+k_{2} & -k_{2} & 0 \\
-k_{2} & k_{2}+k_{3} & -k_{3} \\
0 & -k_{3} & k_{3}
\end{array}\right]\left\{\begin{array}{l}
x_{1} \\
x_{2} \\
x_{3}
\end{array}\right\}=\left\{\begin{array}{c}
k_{1} u+c_{1} \dot{u}+f_{1} \\
0 \\
f_{2}+f_{3}
\end{array}\right\}
\end{gathered}
$$



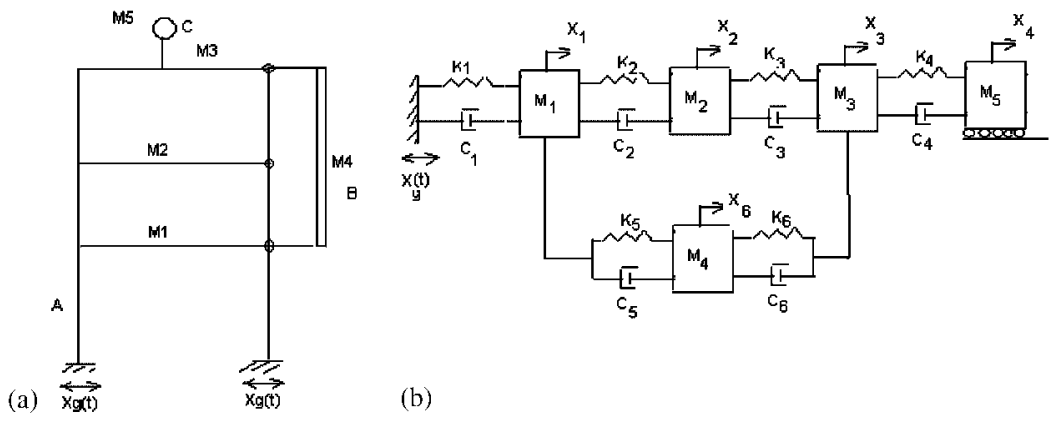

(b)
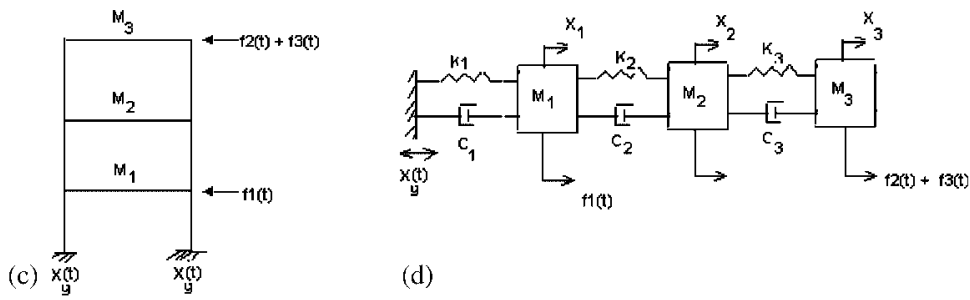

(d)
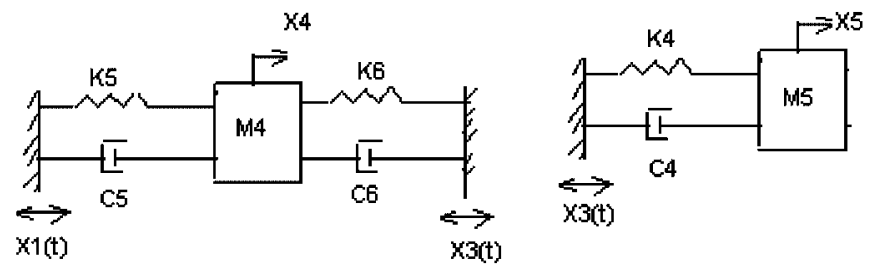

(e)

(f)
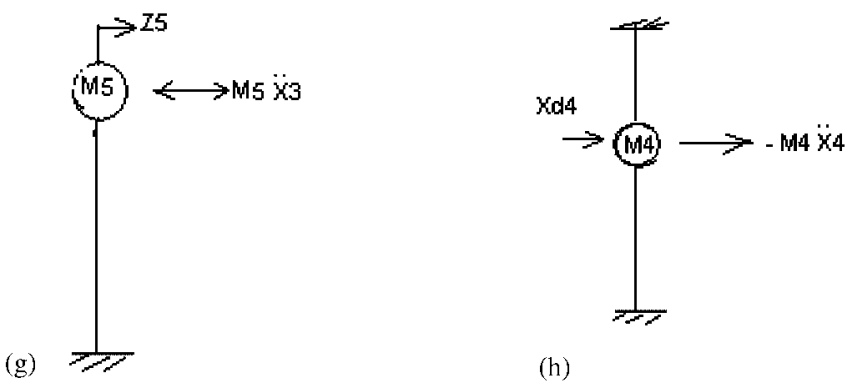

(h)

Figure 16. Example on a 5-dof system subject to support motions: (a) building frame (A) with two subsystems; B: doubly supported subsystem; C: singly supported subsystem; (b) discrete model for the complete system; (c) numerical substructure; (d) model for the numerical substructure; $f_{1}, f_{2}, f_{3}=$ reaction forces that are 'experimentally' measured; (e) test substructure $1 ; x_{1}(t), x_{3}(t)=$ applied support displacements computed from numerical substructure; (f) test substructure $2 ; x_{3}(t)=$ applied support displacements computed from numerical substructure; (g) model for the effective force testing of test substructure 1; and

(h) model for the effective force testing of test substructure 2 . 


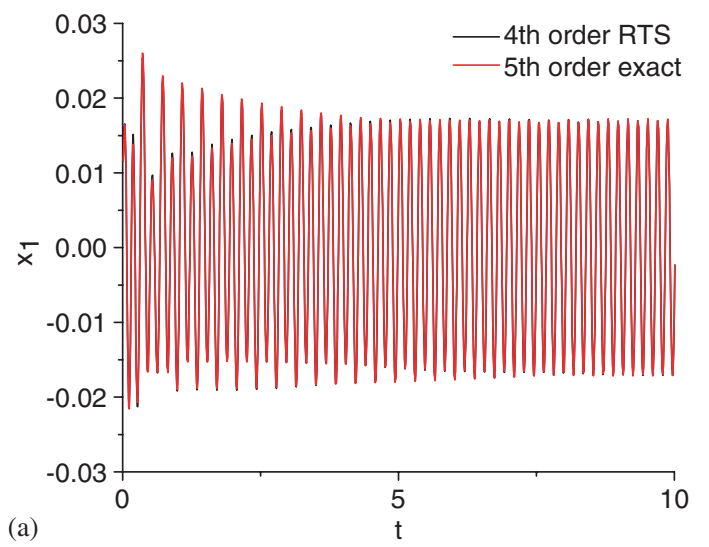

(a)
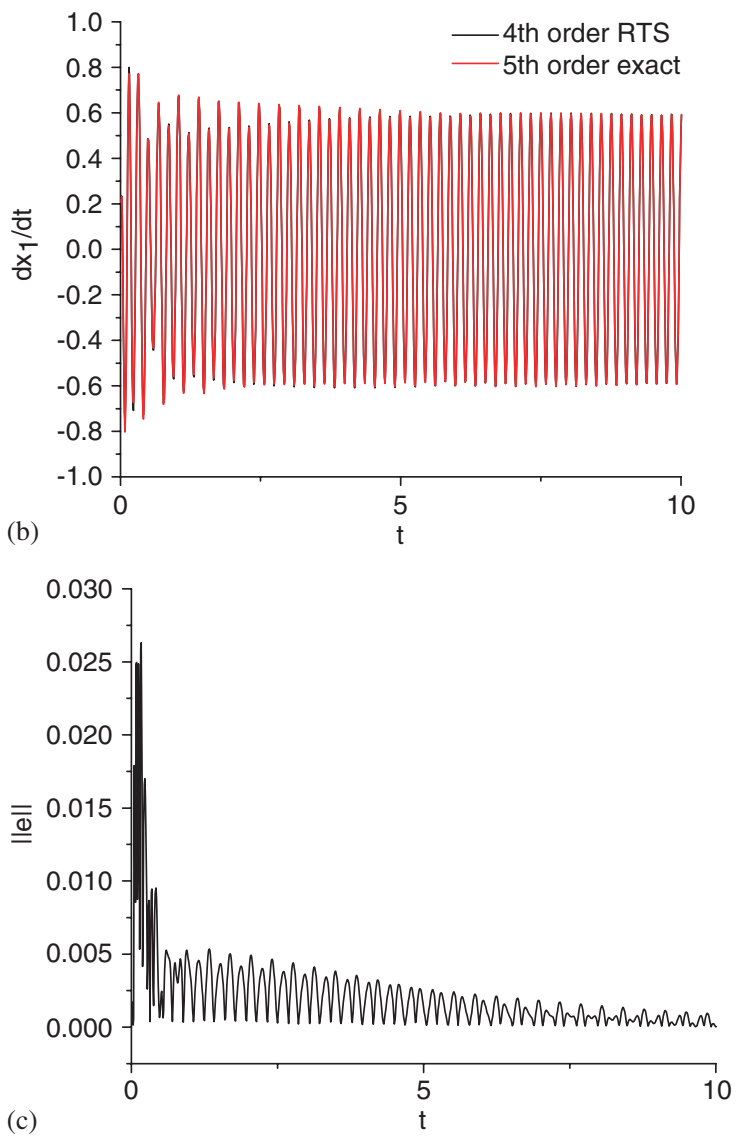

Figure 17. 5-DOF oscillator under sinusoidal support motion: a case of minimal dynamic interactions; $m_{1}=2.0 \mathrm{E} 4, m_{2}=1.5 \mathrm{E} 4, m_{3}=1.2 \mathrm{E} 4, m_{4}=3.0 \mathrm{E} 3, m_{5}=2.5 \mathrm{E} 3, c_{1}=6.959 \mathrm{E} 4, c_{2}=4.6395 \mathrm{E} 4$, $c_{3}=4.6395 \mathrm{E} 4, \quad c_{4}=2.7837 \mathrm{E} 4, \quad c_{5}=3.0157 \mathrm{E} 4, \quad c_{6}=3.0157 \mathrm{E} 4, \quad k_{1}=2.4 \mathrm{E} 7, \quad k_{2}=1.6 \mathrm{E} 7, \quad k_{3}=1.6 \mathrm{E} 7$, $k_{4}=9.6 \mathrm{E} 6, k_{5}=1.04 \mathrm{E} 7, k_{6}=1.04 \mathrm{E} 7, A=0.026, \lambda=35.0$ : (a) history of $x_{1}$; (b) history of $\dot{x}_{1}$; and (c) history of the error norm. 

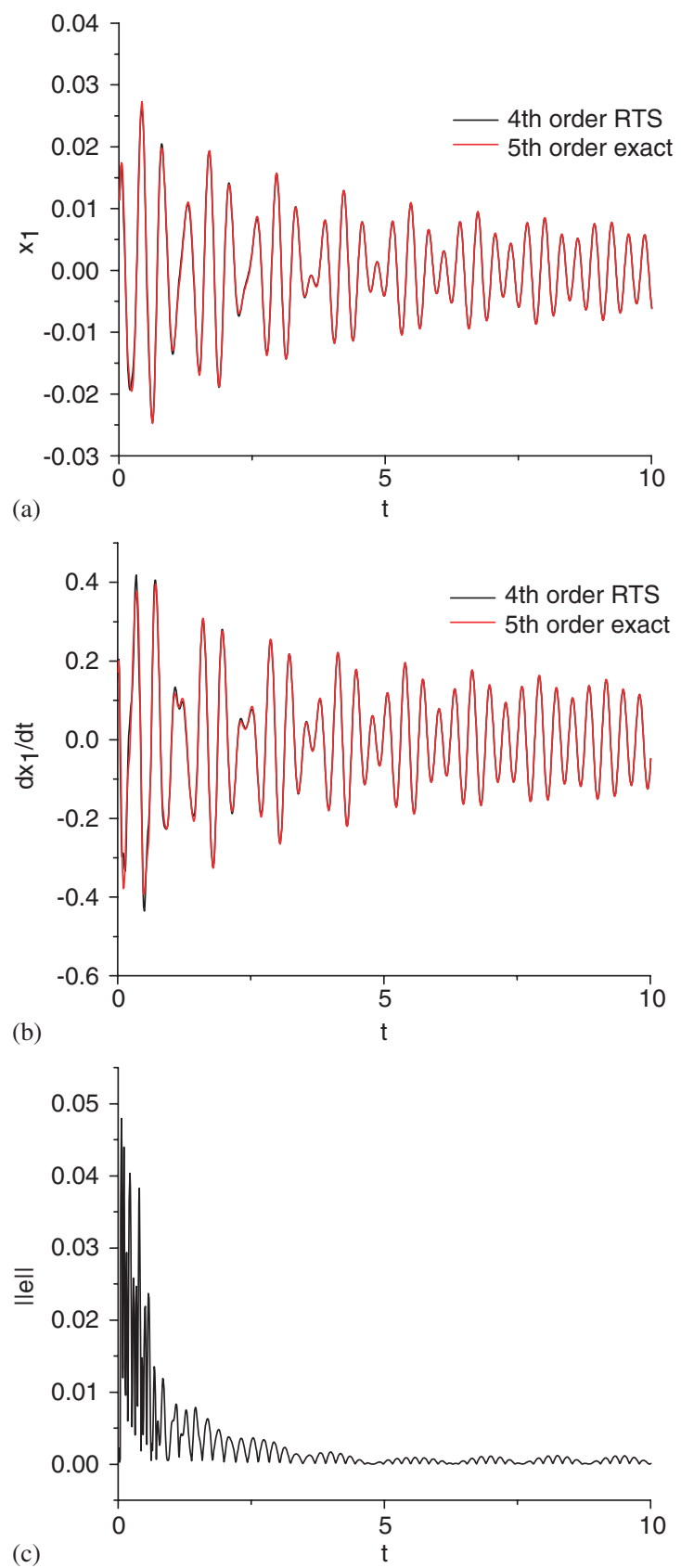

Figure 18. 5-DOF oscillator under sinusoidal support motion: a case of minimal dynamic interactions; $m_{1}=2.4 \mathrm{E} 4, m_{2}=1.5 \mathrm{E} 4, m_{3}=1.2 \mathrm{E} 4, m_{4}=1.0 \mathrm{E} 4, m_{5}=1.0 \mathrm{E} 4, c_{1}=6.959 \mathrm{E} 4, c_{2}=4.6395 \mathrm{E} 4$, $c_{3}=4.6395 \mathrm{E} 4, \quad c_{4}=2.7837 \mathrm{E} 4, \quad c_{5}=3.0157 \mathrm{E} 4, \quad c_{6}=3.0157 \mathrm{E} 4, \quad k_{1}=2.4 \mathrm{E} 7, \quad k_{2}=1.6 \mathrm{E} 7, \quad k_{3}=1.6 \mathrm{E} 7$, $k_{4}=1.6 \mathrm{E} 7, k_{5}=1.6 \mathrm{E} 7, k_{6}=1.6 \mathrm{E} 7, A=0.026, \lambda=20.0$ : (a) history of $x_{1}$; (b) history of $\dot{x}_{1}$; and (c) history of the error norm. 
When the two test substructures are studied in a laboratory, the forces $f_{i}(t), i=1,2,3$ will actually be measured. In the present study, since we are synthetically simulating the behaviour of the test structure, we employ the following expressions for these forces:

$$
\begin{aligned}
& f_{1}(t)=k_{5}\left(x_{4}-x_{1}\right)+c_{5}\left(\dot{x}_{4}-\dot{x}_{1}\right) \\
& f_{2}(t)=k_{4}\left(x_{5}-x_{3}\right)+c_{4}\left(\dot{x}_{5}-\dot{x}_{3}\right) \\
& f_{3}(t)=k_{6}\left(x_{4}-x_{3}\right)+c_{6}\left(\dot{x}_{4}-\dot{x}_{3}\right)
\end{aligned}
$$

Similarly, the determination of the variables $x_{4}(t)$ and $x_{5}(t)$ is based on the equations

$$
\begin{array}{r}
m_{4} \ddot{x}_{4}+c_{5}\left(\dot{x}_{4}-\dot{x}_{1}\right)+c_{6}\left(\dot{x}_{4}-\dot{x}_{3}\right)+k_{5}\left(x_{4}-x_{1}\right)+k_{6}\left(x_{4}-x_{3}\right)=0 \\
m_{5} \ddot{x}_{5}+c_{4}\left(\dot{x}_{5}-\dot{x}_{3}\right)+k_{4}\left(x_{5}-x_{3}\right)=0
\end{array}
$$

Figures 17 and 18 compare results of simulation on the full system (Equation (83)) and the RTSbased solutions (Equations (84)-(86)). Here again, the RTS simulations are observed to perform satisfactorily.

We note that the simulation of test substructures, as per the RTS equations of motion (84)-(86), requires shake tables with RTS simulation capabilities. If test facilities are restricted to the use of only horizontal actuation and reaction wall system, it is still possible to implement the RTS test provided a few assumptions on the nature of the subsystems are considered acceptable. Thus, the equation-governing substructure 2 can be recast as

$$
m_{5} \ddot{z}_{5}+c_{4} \dot{z}_{5}+k_{4} z_{5}=-m_{5} \ddot{x}_{3}
$$

where $z_{5}(t)=x_{5}(t)-x_{3}(t)$ is the relative displacement. The effective force $-m_{5} \ddot{x}_{3}(t)$ can be applied using a horizontal actuation system (Figure 16(g)); this, however, requires the prior knowledge of mass of the subsystem (not a serious restriction), and also, that the subsystem should be such that inertial element can be lumped at one place (restrictive assumption). Similarly, for subsystem 2, if the pseudo-dynamic displacement $x_{4 \mathrm{~s}}(t)$, satisfying the relation $k_{5}\left(x_{4 \mathrm{~s}}-x_{1}\right)+k_{6}\left(x_{4 \mathrm{~s}}-x_{3}\right)=0$, can be experimentally measured, and if damping matrix is taken to be proportional to stiffness, the equation governing the dynamic displacement $x_{4 \mathrm{~d}}(t)=x_{4}(t)-x_{4 \mathrm{~s}}(t)$ can be shown to be given by

$$
m_{4} \ddot{x}_{4 \mathrm{~d}}+\left(c_{5}+c_{6}\right) \dot{x}_{4 \mathrm{~d}}+\left(k_{5}+k_{6}\right) x_{4 \mathrm{~d}}=-m_{4} \ddot{x}_{4 \mathrm{~s}}
$$

This system can be simulated on a test system consisting of reaction wall and horizontal actuation; see Figure 16(h). Here again we are assuming the knowledge of $m_{4}$ and also that the inertial force can be applied as a single concentrated force.

For illustrating the RTS methodology for non-linear structures, we have used the procedure outlined in Section 5.3. Figure 19 shows the non-linear systems considered in this study. Segregations of the systems (as in Figure 19) into numerical and experimental substructures are indicated in Figure 20. The first example (Figures 19(a) and 20(a)) shows a coupling between a linear numerical model and a non-linear experimental model. In the second and third systems (Figures 19(b) and (c)), both the numerical and experimental substructures are non-linear. The excitations in examples 1 and 3 consist of harmonic and earthquake-like support motions. In example 2, on the other hand, we restrict our attention to the undamped free vibration under specified initial conditions. The governing equations of motion for the systems shown in Figures 19(a)-(c) are, 


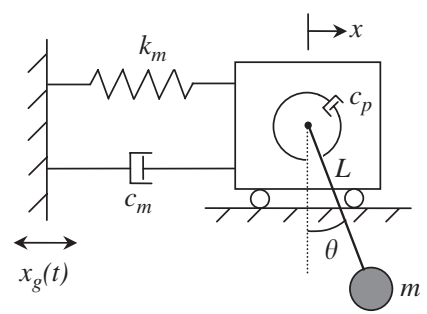

(a)

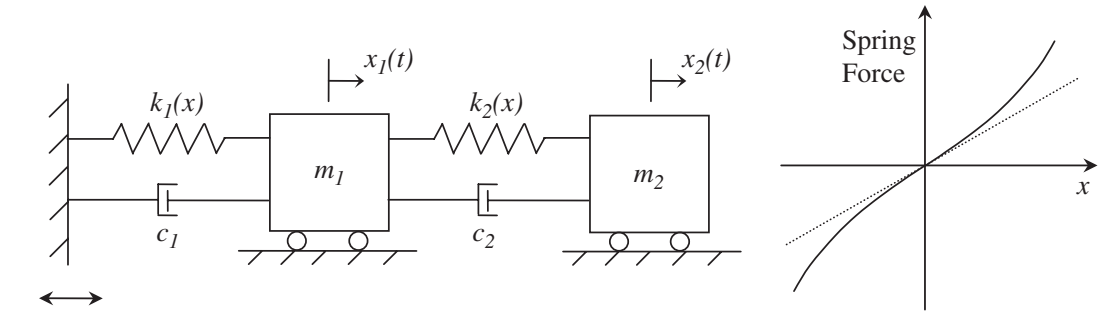

(c) $x_{g}(t)$

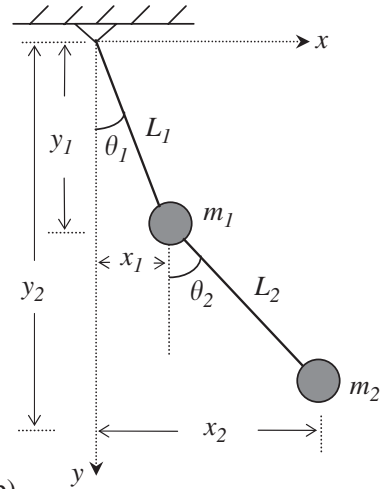

(b)

Figure 19. Non-linear dynamical systems considered in the study: (a) spring-mass pendulum system; (b) double pendulum; and (c) two degree-of-freedom spring-mass system with hardening spring. (Note that, the elastic force in the $i$ th spring is given by $f s_{i}(x)=k_{i}\left(x+\varepsilon_{i} x^{3}\right), i=1,2$.)

respectively, given by

$$
\begin{aligned}
(M+m) \ddot{x}+m L \ddot{\theta} \cos \theta+c_{m} \dot{x}-m L \dot{\theta}^{2} \sin \theta+k_{m} x & =c_{m} \dot{x}_{g}+k_{m} x_{g} \\
m L \ddot{x} \cos \theta+m L^{2} \ddot{\theta}+c_{p} \dot{\theta}+m g L \sin \theta & =0
\end{aligned}
$$

$\left[x\left(t_{0}\right), \dot{x}\left(t_{0}\right), \theta\left(t_{0}\right), \dot{\theta}\left(t_{0}\right)\right]^{\mathrm{T}}=\left[x_{0}, \dot{x}_{0}, \theta_{0}, \dot{\theta}_{0}\right]^{\mathrm{T}} \quad($ system in Figure 19(a))

$$
\begin{gathered}
\left(m_{1}+m_{2}\right) L_{1}^{2} \ddot{\theta}_{1}+m_{2} L_{1} L_{2} \ddot{\theta}_{2} \cos \left(\theta_{1}-\theta_{2}\right)+m_{2} L_{1} L_{2} \dot{\theta}_{2}^{2} \sin \left(\theta_{1}-\theta_{2}\right)+\left(m_{1}+m_{2}\right) g L_{1} \sin \theta_{1}=0 \\
m_{2} L_{2}^{2} \ddot{\theta}_{2}+m_{2} L_{1} L_{2} \ddot{\theta}_{1} \cos \left(\theta_{1}-\theta_{2}\right)-m_{2} L_{1} L_{2} \dot{\theta}_{1}^{2} \sin \left(\theta_{1}-\theta_{2}\right)+m_{2} g L_{2} \sin \theta_{2}=0 \\
{\left[\theta_{1}\left(t_{0}\right), \dot{\theta}_{1}\left(t_{0}\right), \theta_{2}\left(t_{0}\right), \dot{\theta}_{2}\left(t_{0}\right)\right]^{\mathrm{T}}=\left[\theta_{1,0}, \dot{\theta}_{1,0}, \theta_{2,0}, \dot{\theta}_{2,0}\right]^{\mathrm{T}} \quad \text { (system in Figure 19(b)) }}
\end{gathered}
$$

and

$$
\begin{gathered}
m_{1} \ddot{x}_{1}+\left(c_{1}+c_{2}\right) \dot{x}_{1}-c_{2} \dot{x}_{2}+\left(k_{1}+k_{2}\right) x_{1}-k_{2} x_{2}+k_{1} \varepsilon_{1}\left(x_{1}-x_{g}\right)^{3}+k_{2} \varepsilon_{2}\left(x_{1}-x_{2}\right)^{3}=c_{1} \dot{x}_{g}+k_{1} x_{g} \\
m_{2} \ddot{x}_{2}-c_{2} \dot{x}_{1}+c_{2} \dot{x}_{2}-k_{2} x_{1}+k_{2} x_{2}+k_{2} \varepsilon_{2}\left(x_{2}-x_{1}\right)^{3}=0 \\
{\left[x_{1}\left(t_{0}\right), \dot{x}_{1}\left(t_{0}\right), x_{2}\left(t_{0}\right), \dot{x}_{2}\left(t_{0}\right)\right]^{\mathrm{T}}=\left[x_{1,0}, \dot{x}_{1,0}, x_{2,0}, \dot{x}_{2,0}\right]^{\mathrm{T}} \quad(\text { system in Figure 19(c)) }}
\end{gathered}
$$




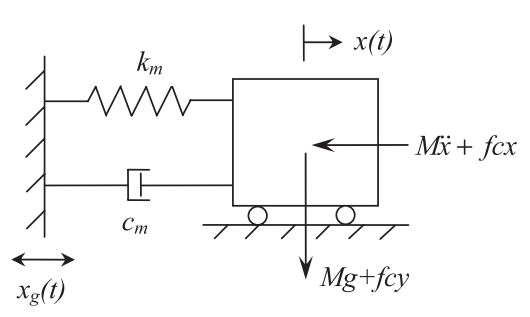

Substructure $S^{I}$

(a)

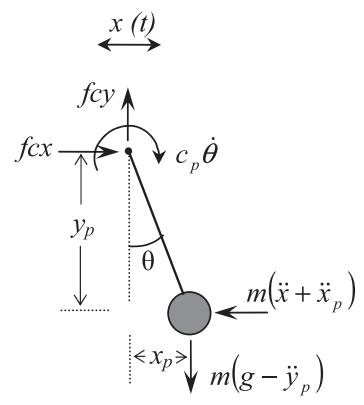

Substructure $S^{I I}$
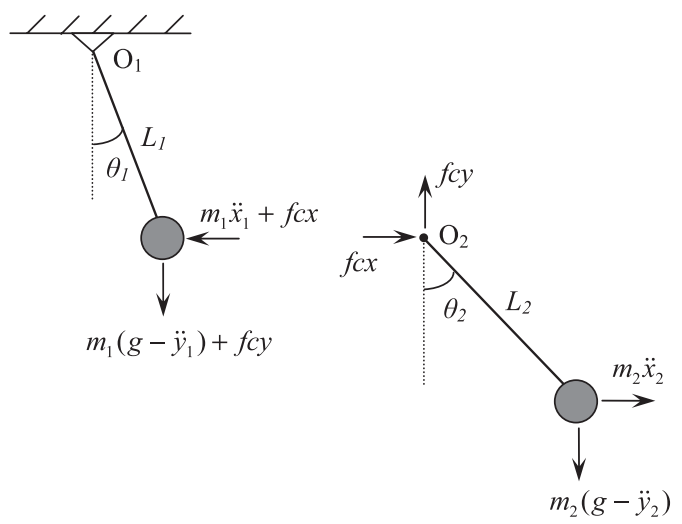

Substructure $S^{I}$

Substructure $S^{I I}$

(b)

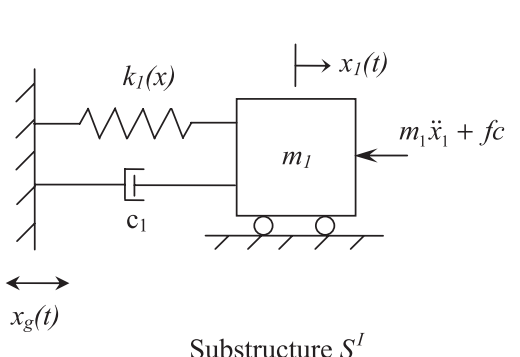

(c)

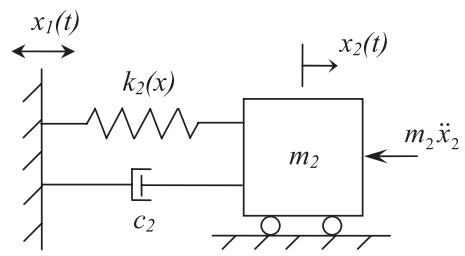

Substructure $S^{I I}$

Figure 20. Non-linear dynamical systems divided into substructures: (a) spring-mass and pendulum interact each other (note that substructure $S^{\mathrm{I}}$ interacts with $S^{\mathrm{II}}$ through the displacement $x_{1}(t)$ and interaction from $S^{\mathrm{II}}$ to $S^{\mathrm{I}}$ is through the forces $f c x$ and $f c y$ ); (b) substructures of double pendulum (here, $f c x$ and $f c y$ represent the interactive forces from $S^{\mathrm{II}}$ to $S^{\mathrm{I}}$. Point $\mathrm{O}_{2}$ of the substructure $S^{\mathrm{II}}$ moves in an angular path described by $\theta_{1}(t)$ and $L_{1}$ ); and (c) substructures of two degree-of-freedom spring-mass system (substructure $S^{\mathrm{I}}$ interacts with $S^{\mathrm{II}}$ through the displacement $x_{1}(t)$ and $S^{\mathrm{II}}$ transmit a force $f c$ to $S^{\mathrm{I}}$ ). 

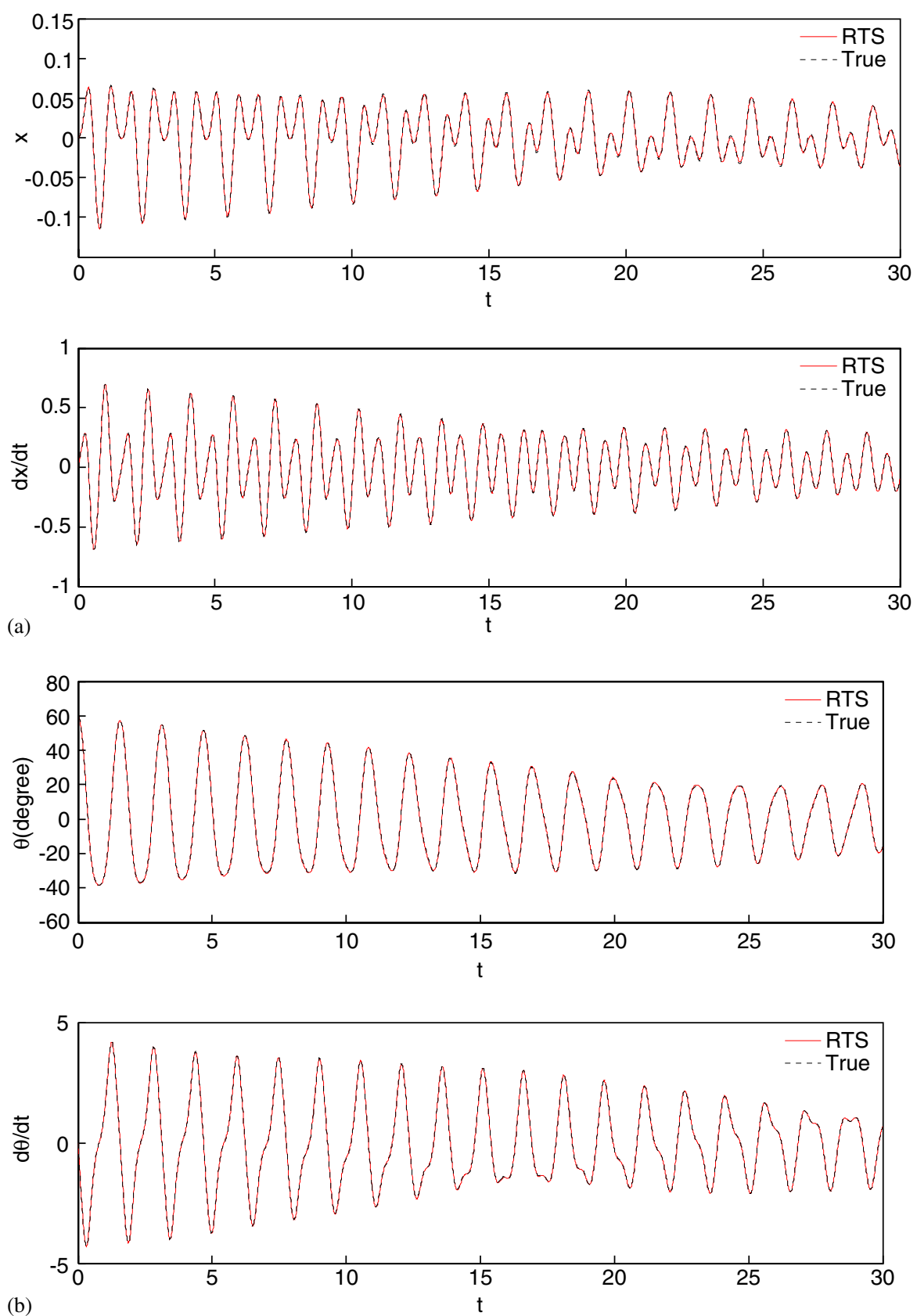

Figure 21. Spring-mass pendulum system subjected to harmonic support motion: (a) time history of $x$ and $\dot{x}$; (b) time history of $\theta$ and $\dot{\theta}$; (c) phase plane plots; (d) Euclidean error norm history (RK-based extrapolation); and (e) Euclidean error norm history (Lagrangian polynomial extrapolation). 

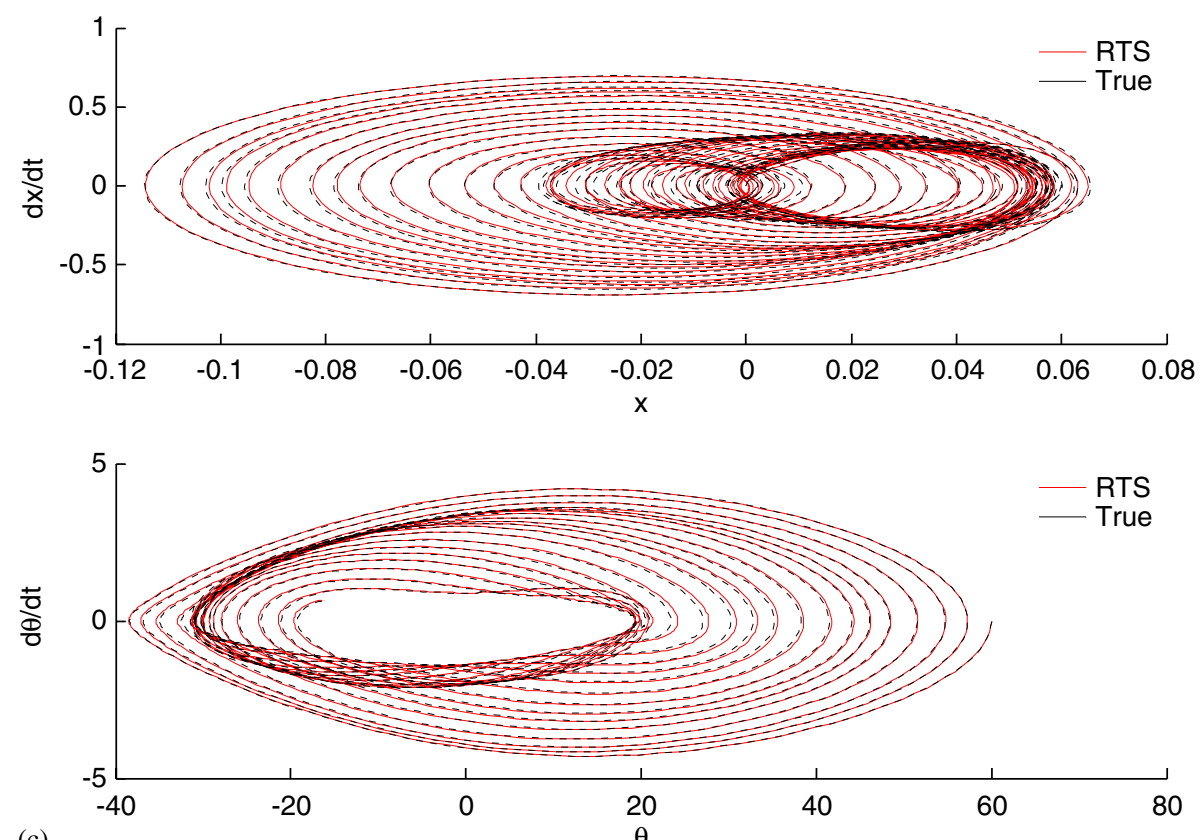

(c)
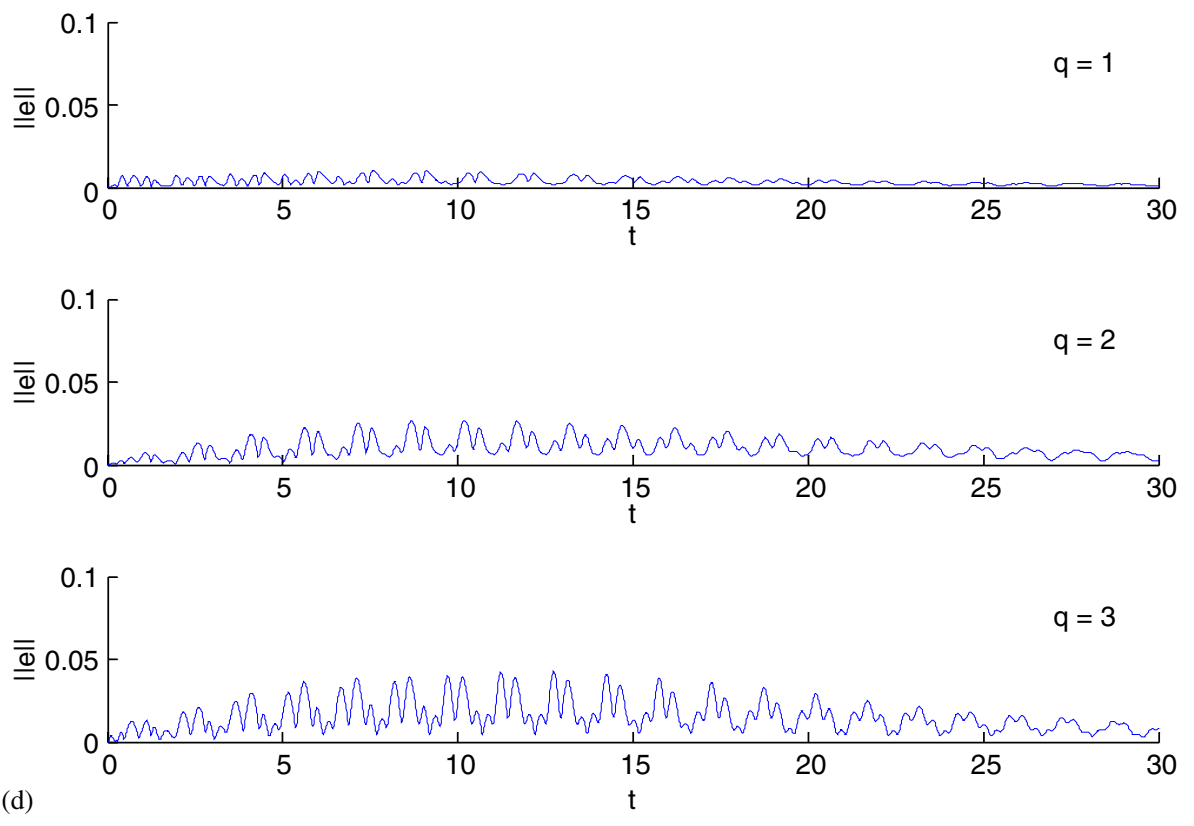

Figure 21. Continued. 

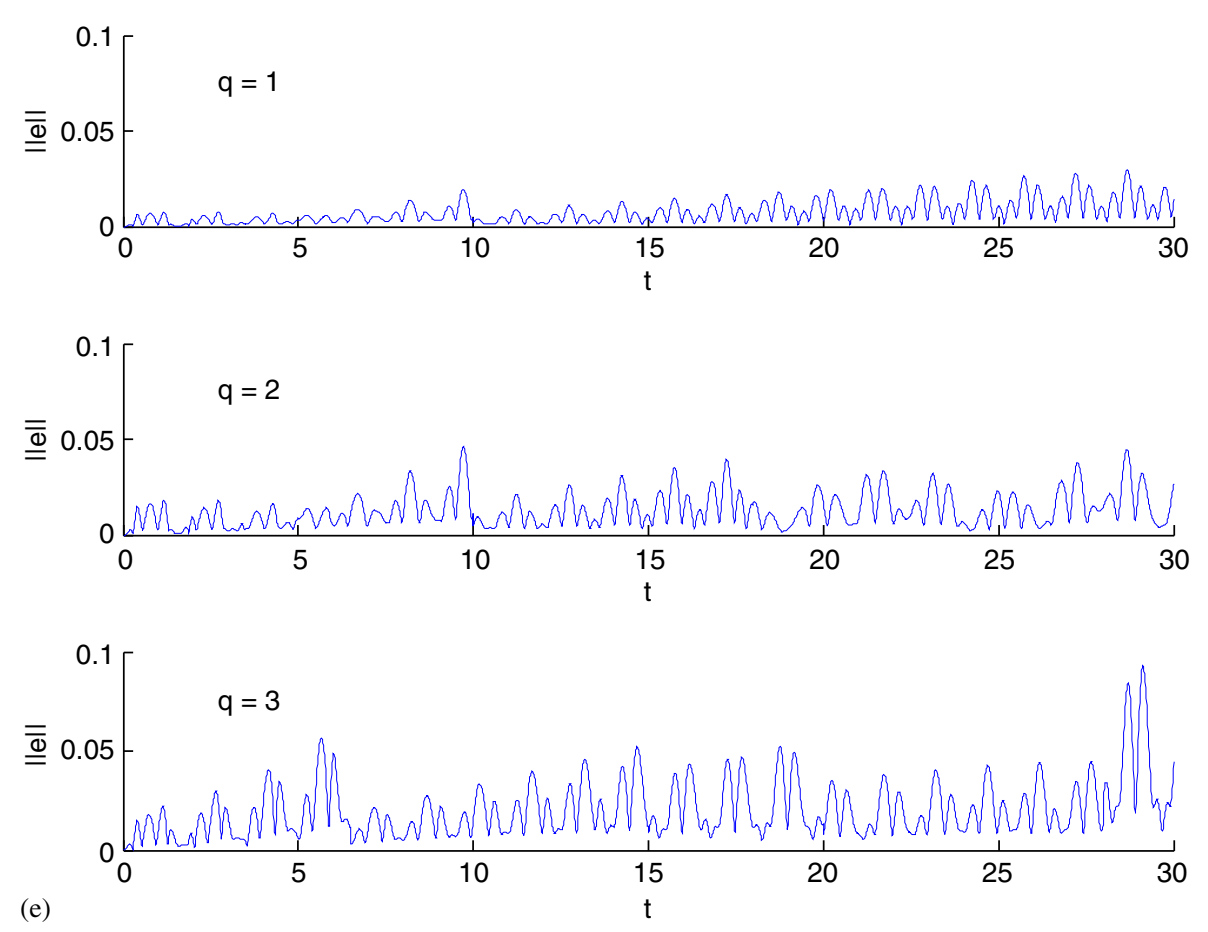

Figure 21. Continued.

Similarly equations suitable for RTS simulation for these structures based on segregations shown in Figure 20 are obtained, respectively, as

$$
\begin{gathered}
M \ddot{x}+c_{m} \dot{x}+k_{m} x=c_{m} \dot{x}_{g}+k_{m} x_{g}-f c x \\
m L^{2} \ddot{\theta}+c_{p} \dot{\theta}+m g L \sin \theta+m \ddot{x} L \cos \theta=0 \\
f c x=m \ddot{x}+m L\left(\ddot{\theta} \cos \theta-\dot{\theta}^{2} \sin \theta\right)
\end{gathered}
$$

$m_{1} L_{1}^{2} \ddot{\theta}_{1}+m_{1} g L_{1} \sin \theta_{1}=-f c x L_{1} \cos \theta_{1}-f c y L_{1} \sin \theta_{1}$

$m_{1} L_{2}^{2} \ddot{\theta}_{2}+m_{2} g L_{2} \sin \theta_{2}=m_{2} L_{1} L_{2}\left[\dot{\theta}_{1}^{2} \sin \left(\theta_{1}-\theta_{2}\right)-\ddot{\theta}_{1} \cos \left(\theta_{1}-\theta_{2}\right)\right]$

$$
\begin{aligned}
& f c x=-m_{2}\left[L_{1}\left(\ddot{\theta}_{1} \cos \theta_{1}-\dot{\theta}_{1}^{2} \sin \theta_{1}\right)+L_{2}\left(\ddot{\theta}_{2} \cos \theta_{2}-\dot{\theta}_{2}^{2} \sin \theta_{2}\right)\right] \\
& f c y=-m_{2}\left(g+\left[L_{1}\left(\ddot{\theta}_{1} \sin \theta_{1}+\dot{\theta}_{1}^{2} \cos \theta_{1}\right)+L_{2}\left(\ddot{\theta}_{2} \sin \theta_{2}+\dot{\theta}_{2}^{2} \cos \theta_{2}\right)\right]\right)
\end{aligned}
$$

and

$$
\begin{aligned}
m_{1} \ddot{x}_{1}+c_{1} \dot{x}_{1}+k_{1} x_{1} & =c_{1} \dot{x}_{g}+k_{1} x_{g}-k_{1} \varepsilon_{1}\left(x_{1}-x_{g}\right)^{3}-f c \\
m_{2} \ddot{x}_{2}+c_{2} \dot{x}_{2}+k_{2} x_{2} & =c_{2} \dot{x}_{1}+k_{2} x_{1}-k_{2} \varepsilon_{2}\left(x_{2}-x_{1}\right)^{3} \\
f c & =k_{2}\left[\left(x_{1}-x_{2}\right)+\varepsilon_{2}\left(x_{1}-x_{2}\right)^{3}\right]+c_{2}\left(\dot{x}_{1}-\dot{x}_{2}\right)
\end{aligned}
$$

In the numerical simulations, the system parameters for example 1, in consistent units, are taken to be $M=15, m=5, L=0.5, c_{m}=0.5, k_{m}=1000$ and $c_{p}=0.1$. Similarly, for example 2, the 

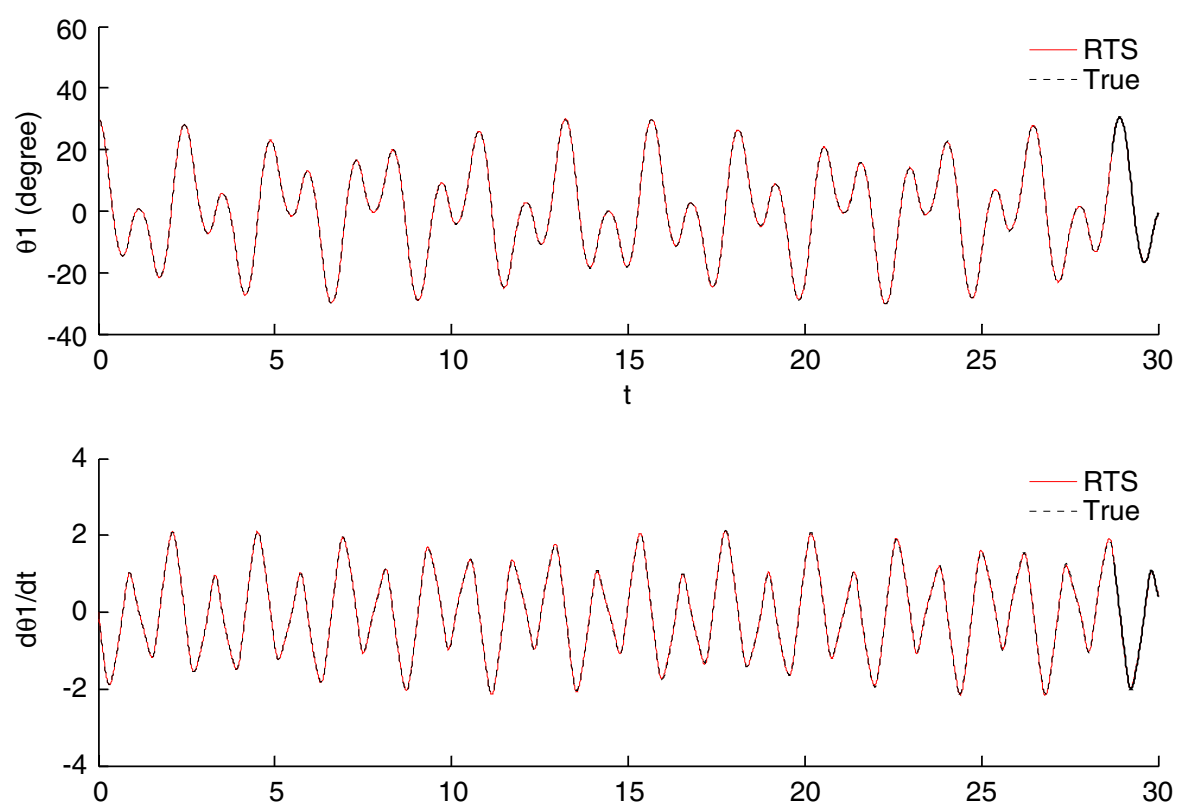

(a)
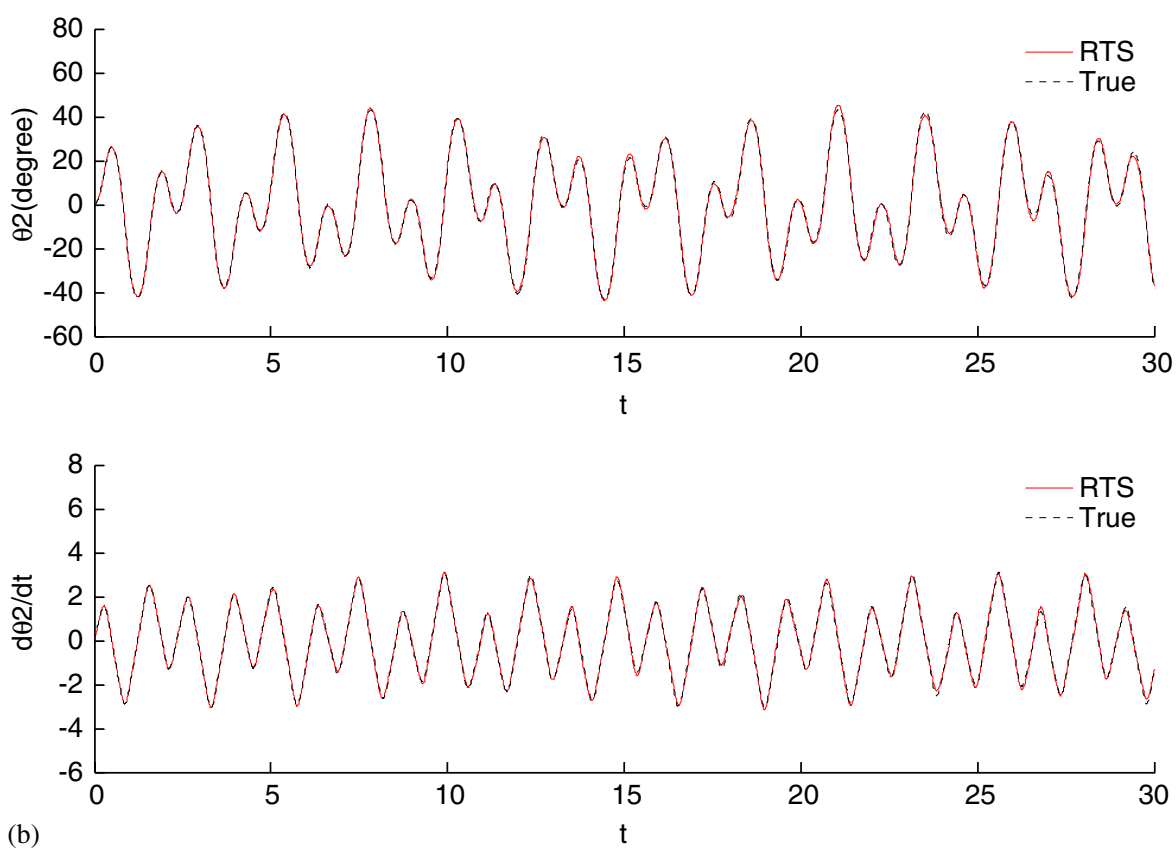

Figure 22. Double pendulum system subjected to the initial conditions: (a) time history of $\theta_{1}$ and $\dot{\theta}_{1}$; (b) time history of $\theta_{2}$ and $\dot{\theta}_{2}$; (c) phase plane plots; and (d) Euclidean error norm history. 

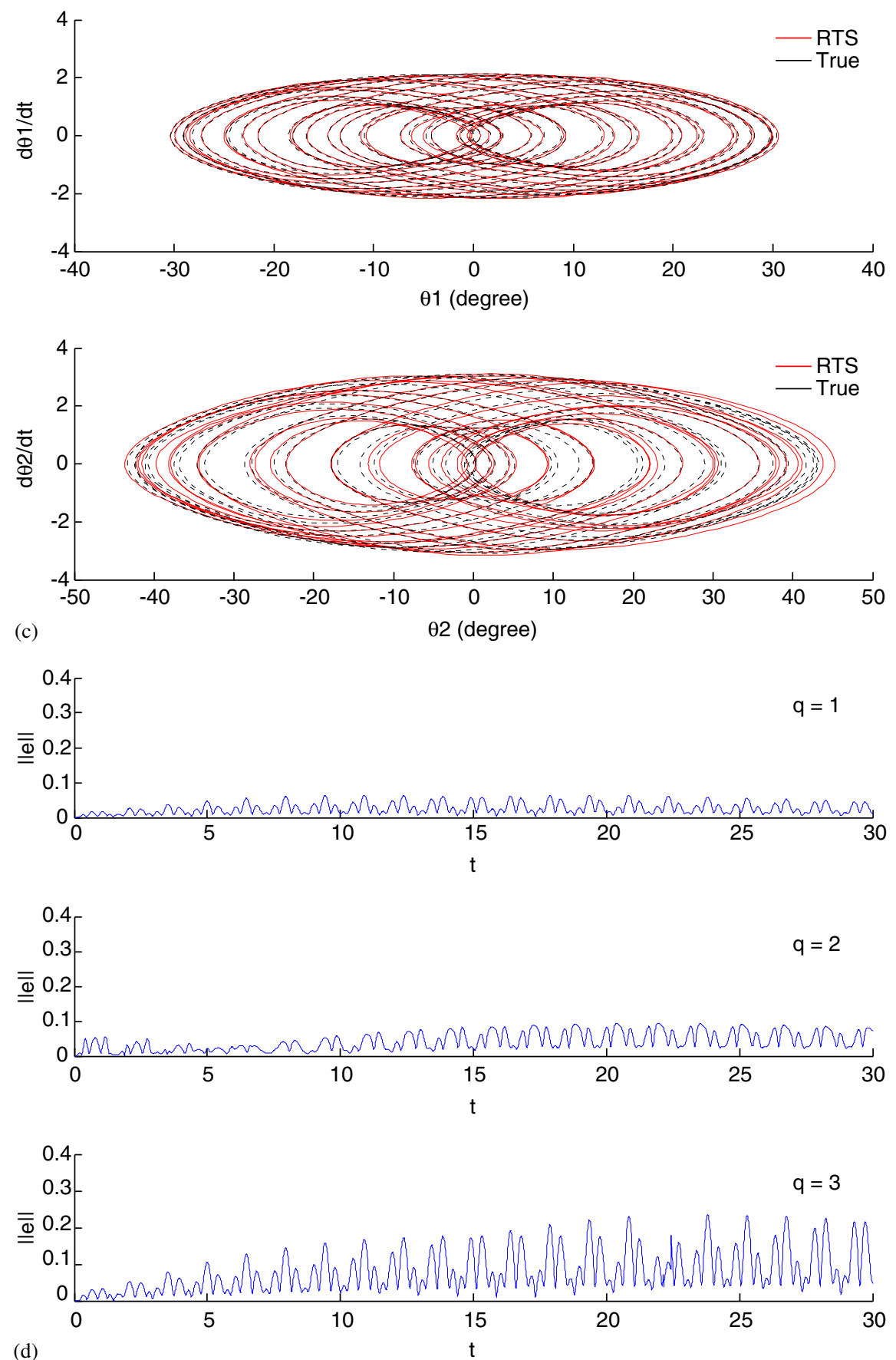

Figure 22. Continued. 

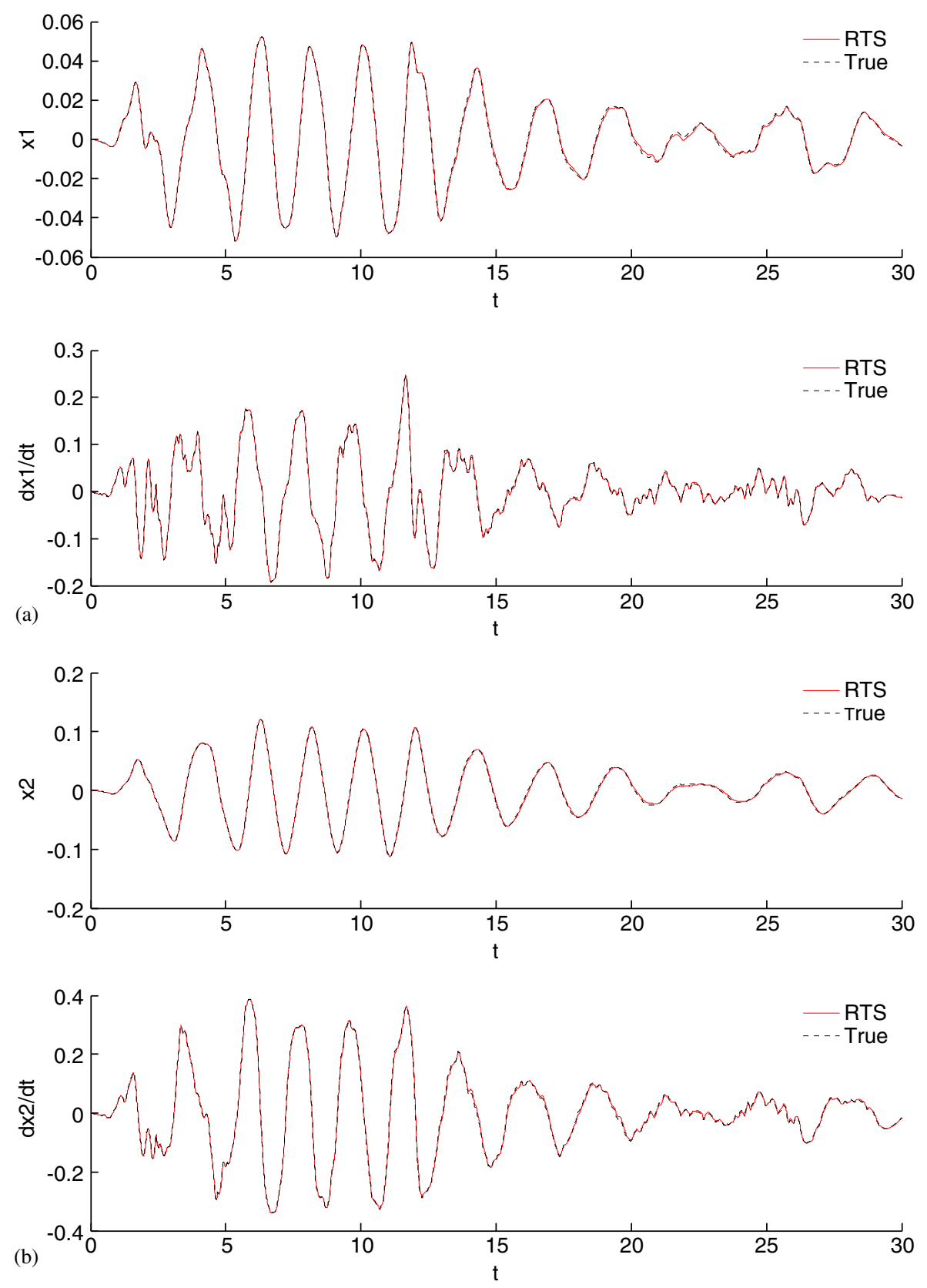

Figure 23. Two degree-of-freedom spring-mass system, with hardening spring, subjected to scaled El Centro ground acceleration: (a) time history of $x_{1}$ and $\dot{x}_{1}$; (b) time history of $x_{2}$ and $\dot{x}_{2}$; (c) phase plane plots; and (d) Euclidean error norm history. 

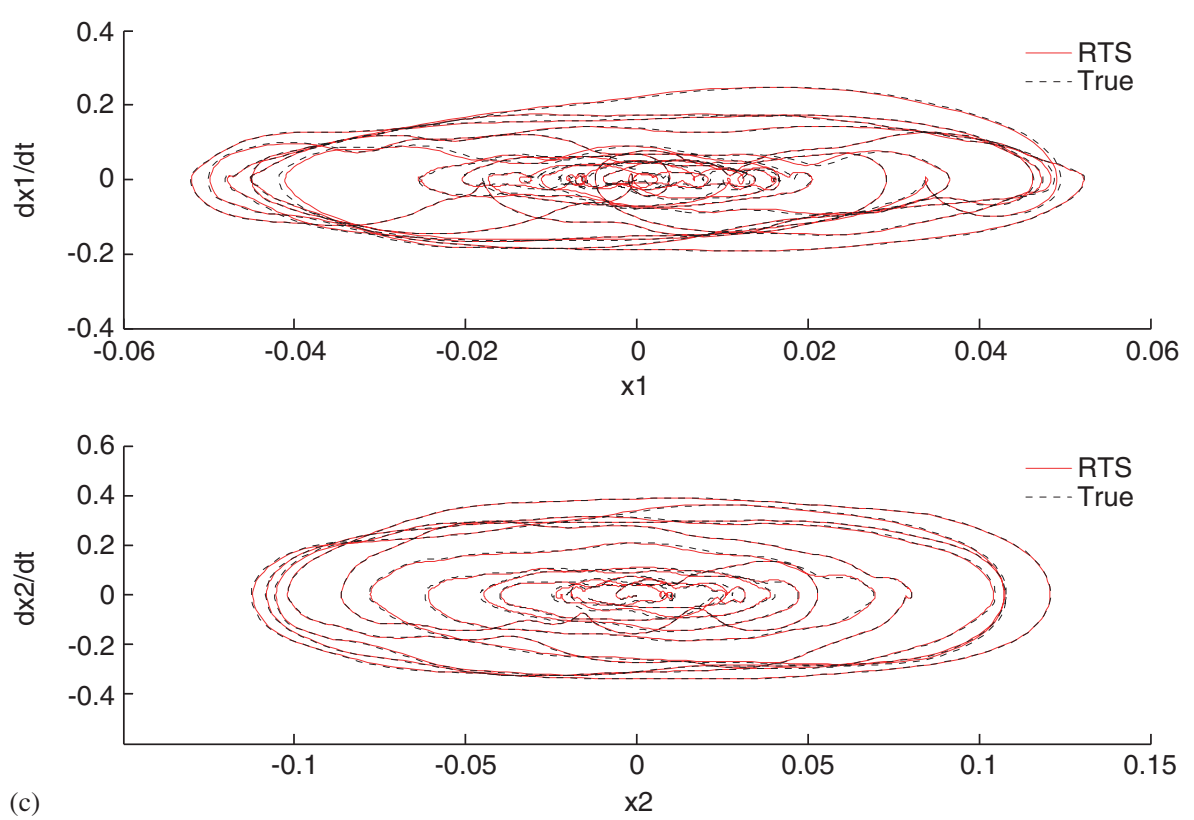

(c)
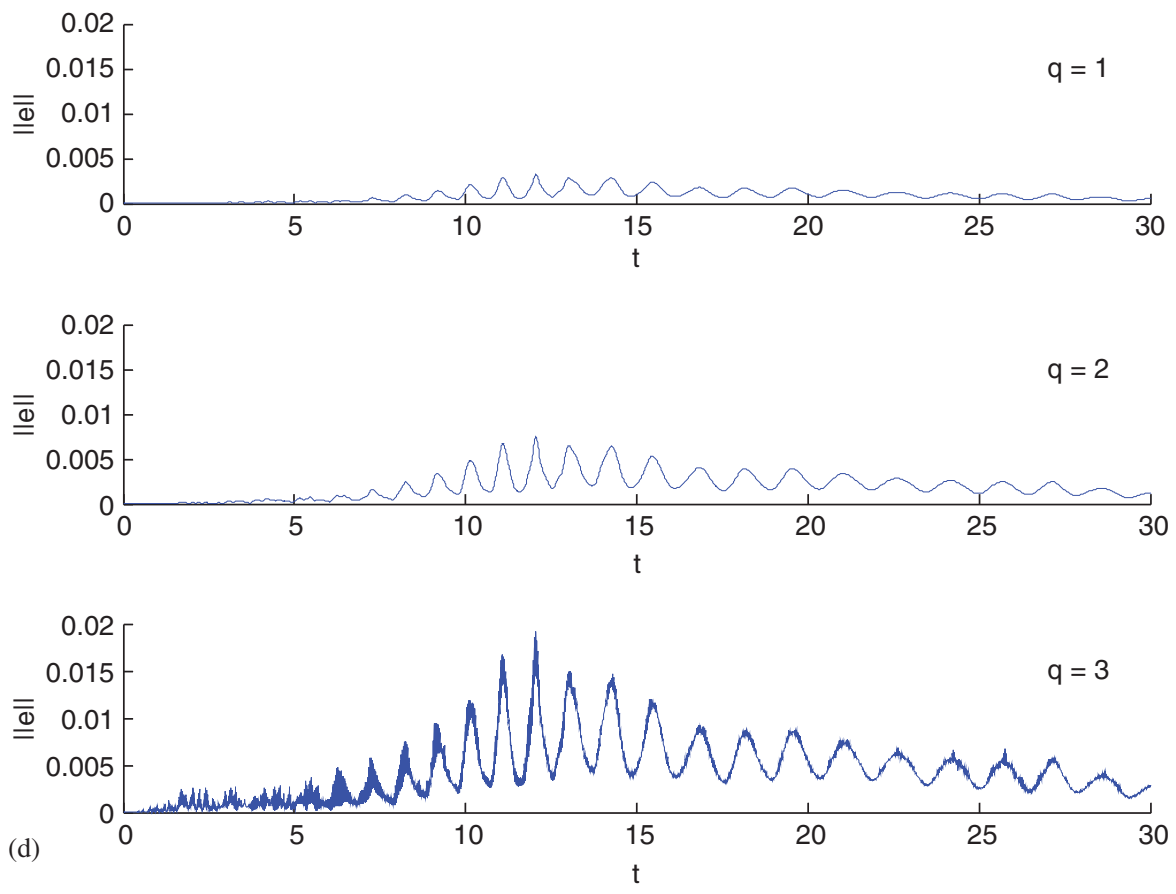

Figure 23. Continued. 
parameters are $m_{1}=1, m_{2}=1, L_{1}=1$ and $L_{2}=1$; and, for example 3, they are $m_{1}=20, m_{2}=15$, $k_{1}=200, k_{2}=100, c_{1}=10, c_{2}=8, \varepsilon_{1}=1000$ and $\varepsilon_{2}=750$. The initial condition vectors are

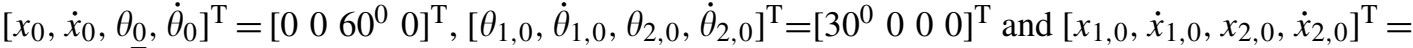
$\left[\begin{array}{llll}0 & 0 & 0 & 0\end{array}\right]^{\mathrm{T}}$, respectively. The harmonic support displacement (example 1) is given by, $x_{g}(t)=x_{g_{0}} \cos$ ( $\lambda t$ ) with parameters $x_{g_{0}}=0.001$ and $\lambda=5$. The support motion considered for example 3 is the El Centro (1940) earthquake ground motion normalized in such a way that the maximum acceleration is 1.25 . For all the three examples, the coupled equations of motion, given by Equations (89)-(91), are solved numerically using a 6th order MTL method $(s=6)$ with $h=0.01$. This solution is treated henceforth as the so-called 'true' solution. In implementing the RTS simulation, the initial phase is taken to end after the integration progresses by nine time steps. In implementing the RKbased extrapolation method, the cubic spline, as in Equation (60), is used as the kernel function. The RTS-based solution is obtained for $q=1,2$ and 3 choosing a constant value of $p=10$. It may be noted that the RTS simulations using RK-based multi-step extrapolation with $q=2$ and 3 can handle time delays of magnitudes up to $2 h$ and $3 h$, respectively. This may be contrasted with the single-step extrapolation scheme $(q=1)$ that can handle time delays up to $h$ only. For all the simulations, the history of the instantaneous error norm is calculated as $\|e(t)\|=\left\|Y_{\text {true }}(t)-Y_{\text {rts }}(t)\right\|$, where $Y_{\text {true }}$ refers to the response of the coupled system obtained through a direct integration and $Y_{\text {rts }}$ refers to the response obtained using the proposed RTS procedure. For comparisons with the so-called true response, the response obtained via the RTS procedure with $q=3$ is considered for all the examples presented here.

The results of RTS simulations of the three non-linear systems are shown in Figures 21-23. These include time histories of state variables (Figures 21(a), (b), 22(a), (b), 23(a) and (b)), phase plane plots (Figures 21(c), 22(c) and 23(c)) and plots of error norms (Figures 21(d), 22(d) and 23(d)). In all the cases, simulations are based on the RK-based extrapolation. The system considered in example 1 is, however, analysed using both ILP- and RK-based simulations. Figure 21(e) shows the plots of error norms based on the ILP method.

From Figures 21 to 23 it is observed that the RTS and true solution show acceptable mutual agreement. It is evident that the RTS simulations have the potential to successfully retain the pathwise fidelity to the true solution during both the iterative and non-iterative phases. In particular, it is of interest to note that the RTS simulations correctly capture the sensitivity of paths to specified initial conditions. Furthermore, the errors do not show any systematic growth in time, thereby, indicating that the solution strategy does not lead to an undesirable growth of local errors as time

progresses. This is true for response under harmonic forces (Figure 21), undamped free vibration (Figure 22) and transient aperiodic excitation (Figure 23). As expected, the supremum of error in all the three examples increases as $q$ increases from 1 to 3 in the RK extrapolation. The superior performance of the RK-based RTS simulations over the ILP-based simulation is evident from Figures 21(d) and (e). Thus, the highest error norm in the RK-based simulation is found to be 0.0425 with $q=3$ (Figure 21(d)), while the same quantity in the ILP-based simulation reaches a value of 0.0934 (Figure 21(e)).

\section{CONCLUDING REMARKS}

A new form of the real time sub-structuring (RTS) method is proposed in the context of linear/nonlinear structural dynamics. Using this, a semi-discretized structural dynamical system may be decomposed into a combination of an arbitrary number of 'numerical' and 'test' substructures so 
that their component solutions may be suitably assembled to recover the solution of the entire dynamical system in a path-sensitive manner and, in principle, to a high order of accuracy. If the dynamical response of a substructure is obtained through numerical or analytical means, then it is referred to as a 'numerical' substructure. In case numerical solution for a substructure is found to be difficult or impracticable (probably owing to the lack of a sufficiently accurate mathematical model or some other reason), then it is also possible to determine the sub-structural response through a laboratory experiment and, in such a case, the substructure is referred to as a 'test' substructure.

In order to preserve possibly history-dependent behaviour of some structural systems, the RTS methodology is developed in such a way that the numerical substructures dynamically interact amongst themselves and with the test substructures in real time. These interactions are modelled, within the RTS framework, through a set of interacting forcing functions and they may be constructed, for a given substructure, using interpolation/extrapolation schemes based on the knowledge of the response of the dynamical system at previous time instants. However, while starting the RTS-based simulations and/or experiments, only the initial conditions are known and this is generally not enough to construct the interactive forces with sufficient accuracy. This problem during start-up has presently been avoided through an interactive procedure, which has been shown to monotonically converge to the targeted path-wise solution with an increasing number of iterations. The method of numerical sub-structuring reported in this study provides a continuous approximation to the response of the substructure. A continuous approximation is particularly useful as it helps tackle aliasing errors even under relatively large time step sizes and thus provides a better insight into the response behaviour. Furthermore, in the context of linear time-invariant substructures, the study also discusses the possibility of numerically dissipating high-frequency noise in the integration process. For such cases, an implicit scheme for integration of the numerical substructure is proposed and the stability properties of this scheme are investigated. For non-linear substructures, the governing differential equations are solved using an integration scheme based on MTL method. In the process, the order of accuracy of the numerical simulation can be increased with little extra effort and the need for a numerical differentiation of the non-linear functions is avoided at all stages. To extrapolate the interactive forces/displacements, a reproducing kernel (RK)-based extrapolation technique is proposed, which is found to be superior to Lagrangian polynomial extrapolation. The RK-based extrapolation scheme is attractive in situations that demand the interaction effects to be predicted over multiple time steps ahead.

Finally, we note that the proposed technique of numerical sub-structuring may be implemented within a parallel computing environment, wherein each numerical substructure may be independently integrated using a separate processor. This particular observation may be suitably exploited in applying the RTS method for very large-dimensional numerical substructures. In the numerical experiments presented in this paper, the measurements are assumed to be noise free. A future work, believed to be of immense practical significance, would be to devise a means to handle noise in the RTS simulations and thus, hopefully, enable the authors to experimentally validate the proposed method.

\section{ACKNOWLEDGEMENTS}

Financial support from the Indian Institute of Science under the tenth plan grant is thankfully acknowledged by the authors. 


\section{REFERENCES}

1. Bathe KJ. Finite Element Procedures. Prentice-Hall: New Delhi, 1996.

2. Ewins DJ. Modal Testing: Theory, Practice and Application. Research Studies Press Ltd: Baldock, 2000.

3. Maia NMM, Silva JMM. Theoretical and Experimental Modal Analysis. Research Studies Press Ltd: Taunton, 1997.

4. Williams MS, Blakeborough A. Laboratory testing of structures under dynamic loads: an introductory review. Philosophical Transactions of the Royal Society of London, Series A 2001; 359:1651-1669.

5. Takanashi K, Nakashima M. Japanese activities on on-line testing. Journal of Engineering Mechanics (ASCE) 1987; 113(7):1014-1032.

6. Severn RT, Brownjohn JMW, Dumanoglu AA, Taylor CA. A review of dynamic testing methods for civil engineering structures. Proceedings of the Conference on Civil Engineering Dynamics, University of Bristol, $1989 ; 1-24$.

7. Nakashima M. Development, potential, and limitations if real-time online (pseudo-dynamic) testing. Philosophical Transactions of the Royal Society of London, Series A 2001; 359:1851-1867.

8. Krawinkler H. Guest editor, special issue: applications of experimental techniques. ISET Journal of Earthquake Engineering 2000; 37(4):47-197.

9. Mahin SA, Shing PB. Pseudodynamic method for seismic testing. Journal of Structural Engineering (ASCE) 1985; 111(7):1482-1503.

10. Mahin SA, Shing PB, Thewalt CR, Hanson RD. Pseudodynamic test method-current status and future directions. Journal of Structural Engineering (ASCE) 1989; 115:2113-2128.

11. Dimig J, Shield C, French C, Bailey F, Clark A. Effective force testing: a method for seismic simulation for structural testing. Journal of Structural Engineering (ASCE) 1999; 125(9):1028-1037.

12. Shield CK, French CW, Timm J. Development and implementation of the effective force testing method for seismic simulation of large-scale structures. Philosophical Transactions of the Royal Society of London, Series A 2001; 359:1911-1929.

13. Kausel E. New seismic testing method. I: Fundamental concepts. Journal of Engineering Mechanics (ASCE) 1996; 124(5):565-570.

14. Kausel E. New seismic testing method. II: Proof for MDOF systems. Journal of Engineering Mechanics (ASCE) 1996; 124(5):571-575.

15. Shing PB, Nakashima M, Bursi OS. Application of pseudodynamic test method to structural research. Earthquake Spectra 1996; 12:29-56.

16. Donea J, Magnonette G, Negro P, Pegon P, Pinto A, Verzeietti G. Pseudodynamic testing capabilities of the ELSA laboratory for earthquake testing of large structures. Earthquake Spectra 1996; 12(1):163-180.

17. Nakashima M, Kato H, Takaoka E. Development of real-time pseudo dynamic testing. Earthquake Engineering and Structural Dynamics 1992; 21:79-92.

18. Nakashima M, Masaoka N. Real-time on-line test for MDOF systems. Earthquake Engineering and Structural Dynamics 1999; 28:393-420.

19. Horiuchi T, Inoue M, Konno T, Namita Y. Real-time hybrid experimental system with actuator delay compensation and its application to piping system with energy absorber. Earthquake Engineering and Structural Dynamics 1999; 28:1121-1141.

20. Horiuchi T, Konno T. A new method for compensating for actuator delay in real-time hybrid experiments. Philosophical Transactions of the Royal Society of London 2001; 359:1893-1909.

21. Wagg DJ, Stoten DP. Substructuring of dynamical system via the adaptive minimal control synthesis algorithm. Earthquake Engineering and Structural Dynamics 2001; 30:859-877.

22. Darby PA, Blakeborough A, Williams MS. Improved control algorithm for real-time substructuring. Earthquake Engineering and Structural Dynamics 2001; 30:431-448.

23. Blakeborough A, Williams MS, Darby AP, Williams DM. The development of real-time substructure testing. Philosophical Transactions of the Royal Society of London 2001; 359:1869-1891.

24. Zhao J, French C, Shield C, Posbergh T. Consideration for the development of real-time dynamic testing using servo-hydraulic actuation. Earthquake Engineering and Structural Dynamics 2003; 32:1773-1794.

25. Pan P, Nakashima M, Tomofuji HT. Online test using displacement-force mixed control. Earthquake Engineering and Structural Dynamics 2005; 34:869-888.

26. Neild SA, Stoten DP, Drury D, Wagg DJ. Control issues relating to real-time substructuring experiments using a shake table. Earthquake Engineering and Structural Dynamics 2005; 34:1171-1192. 
27. Wallace MI, Seiber J, Neild SA, Wagg DJ, Krauskopf B. Stability analysis of real-time dynamic substructuring using delay differential models. Earthquake Engineering and Structural Dynamics 2005; 34:1817-1832.

28. Wallace MI, Wagg DJ, Neild SA. An adaptive polynomial based forward prediction algorithm for multi-actuator real-time dynamic substructuring. Proceedings of the Royal Society of London, Series A 2005; 461:3807-3826.

29. Wu B, Xu G, Wang Q, Williams MS. Operator-splitting for real-time substructure testing. Earthquake Engineering and Structural Dynamics 2006; 35:293-314.

30. Wang T, Nakashima M, Pan P. On-line hybrid test combining general purpose finite element software. Earthquake Engineering and Structural Dynamics 2006; 35:1471-1488.

31. Kyrychko YN, Blyuss KB, Gonzalez-Buelga A, Hogan SJ, Wagg DJ. Real-time dynamic substructuring in a coupled oscillator-pendulum system. Proceedings of the Royal Society of London, Series A 2006; 462:1271-1294.

32. Thewalt CR, Mahin SA. An unconditionally stable hybrid pseudodynamic algorithm. Earthquake Engineering and Structural Dynamics 1995; 24:723-731.

33. Combescure D, Pegon P. $\alpha$-operator splitting time integration technique for pseudodynamic testing error propagation analysis. Soil Dynamics and Earthquake Engineering 1997; 16:427-443.

34. Chang SY. Application of the momentum equations of motion to pseudo-dynamic testing. Philosophical Transactions of the Royal Society of London, Series A 2001; 359:1801-1827.

35. Wu B, Bao H, Ou J, Williams MS. Stability and accuracy analysis of the central difference method for real-time substructure testing. Earthquake Engineering and Structural Dynamics 2005; 34:705-718.

36. Roy D, Kumar R. A multistep transversal linearization (MTL) method in nonlinear structural dynamics. Journal of Sound and Vibration 2005; 287:203-226.

37. Roy D, Ramachandra LS. A generalized local linearization principle for non-linear dynamical systems. Journal of Sound and Vibration 2001; 41:653-679.

38. Roy D, Ramachandra LS. A semi-analytical locally transversal linearization method for non-linear dynamical systems. International Journal for Numerical Methods in Engineering 2001; 51:203-224.

39. Chen JS, Pan C, Wu CT, Liu WK. Reproducing kernel particle methods for large deformation analysis of nonlinear structures. Computer Methods in Applied Mechanics and Engineering 1996; 139:195-229.

40. Li S, Liu WK. Meshfree and particle methods and their applications. Applied Mechanics Review 2002; 55:1-34.

41. Saito N, Beylkin G. Multi-scale representations using autocorrelation functions of compactly supported wavelets. IEEE Transactions on Signal Processing 1993; 41:3584-3590.

42. Hoffman DK, Wei GW, Zhang DS, Kouri DJ. Shannon-Gabor, wavelet distributed approximating functional. Chemical Physics Letters 1998; 287:119-124.

43. Roy D. A numeric-analytic technique for non-linear deterministic and stochastic dynamical systems. Proceedings of the Royal Society of London, Series A 2001; 457:539-566.

44. Hughes TJR. The Finite Element Method. Prentice-Hall: Englewood Cliffs, NJ, 1987.

45. Romero I. On the stability and convergence of fully discrete solutions in linear elastodynamics. Computer Methods in Applied Mechanics and Engineering 2002; 191:3857-3882.

46. Romero I. Stability analysis of linear multistep methods for classical elastodynamics. Computer Methods in Applied Mechanics and Engineering 2004, in press.

47. Darby PA, Williams MS, Blakeborough A. Stability and delay compensation for real-time substructure testing. Journal of Engineering Mechanics (ASCE) 2002; 128(12):1276-1284.

48. Liu WK, Jun S, Li S, Adee J, Belytschko T. Reproducing kernel particle methods for structural dynamics. International Journal for Numerical Methods in Engineering 1995; 38:1655-1679.

49. Liu WK, Jun S, Zhang YF. Reproducing kernel particle methods. International Journal for Numerical Methods in Fluids 1995; 20:1081-1106.

50. Liu WK, Han W, Lu H, Li S, Cao J. Reproducing kernel element method. Part I: theoretical formulation. Computer Methods in Applied Mechanics and Engineering 2004; 193:933-951.

51. Chen JS, Han W, You Y, Meng X. A reproducing kernel method with nodal interpolation property. International Journal for Numerical Methods in Engineering 2003; 56:935-960.

52. Aluru NR. A point collocation method based on reproducing kernel approximations. International Journal for Numerical Methods in Engineering 2000; 47:1083-1121.

53. Shaw A, Roy D. A NURBS-based error reproducing kernel method (ERKM) applied to non-linear mechanics. Computational Mechanics 2006, in press.

54. Chen Y, Soong TT. Seismic response of secondary systems, state-of-the-art review. Engineering Structures 1988; 10:218-228. 